\title{
Gram-Scale, Seven-Step Total Synthesis of (-)-Colchicine
}

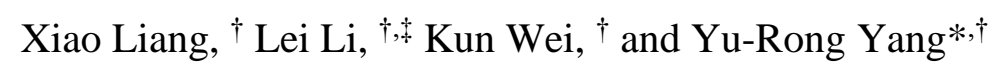 \\ 'State Key Laboratory of Phytochemistry and Plant Resources in West China, Kunming Institute of Botany, \\ Chinese Academy of Sciences, Kunming 650201, China University of Chinese Academy of Sciences, Beijing \\ 100049, China
}

Email: yangyurong@mail.kib.ac.cn

\section{Table of Contents}

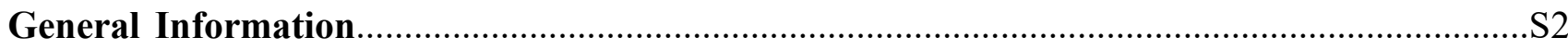

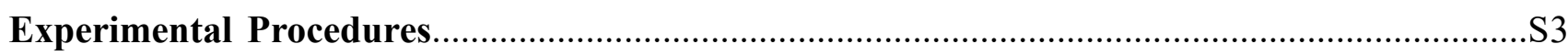

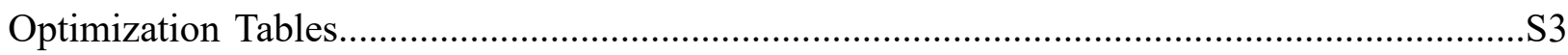

Synthetic Procedures and Characterization Data................................................................. 9

Comparison of NMR data for Commercial vs. Synthetic (-)-Colchicine...........................S18

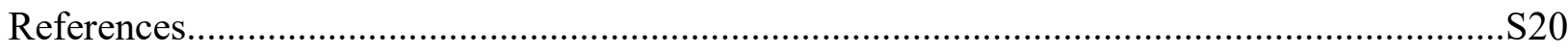

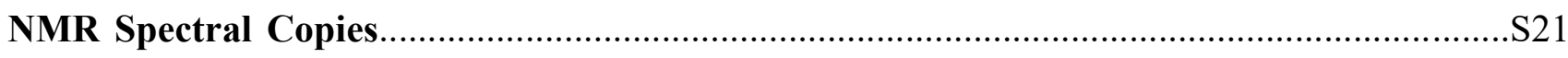

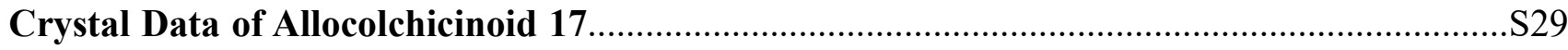

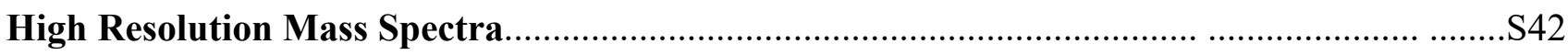




\section{General Information}

Unless otherwise stated, all oxygen or moisture sensitive reactions were conducted in flame-dried glassware under an atmosphere of nitrogen. All solvents were purified and dried according to standard methods prior to use. (S)-L and ( $r a c)-\mathbf{L}$ were prepared according to the reported procedure. ${ }^{1}$ Reagents were purchased from commercial sources and were used without further purification.

Chromatographic purification of products was accomplished using forced-flow chromatography on 200-300 mesh silica gel supplied by Tsingtao Haiyang Chemicals (China). The TLC glass plates were performed on $0.20 \mathrm{~mm}$ silica gel GF254 plates supplied by Yantai Chemicals (China), and visualized with UV light ( $254 \mathrm{~nm}$ ), exposure to iodine vapor, or basic aqueous potassium permanganate $\left(\mathrm{KMnO}_{4}\right)$.

${ }^{1} \mathrm{H}$ and ${ }^{13} \mathrm{C}$ NMR spectra were acquired on Bruker Avance III 400 and 600 NMR spectrometer. Chemical shifts were given in parts per million (ppm) with reference to residual solvent signals $\left[{ }^{1} \mathrm{H}\right.$ NMR: $\mathrm{CDCl}_{3}$ (7.26), ${ }^{13} \mathrm{C}^{\mathrm{NMR}}: \mathrm{CDCl}_{3}$ (77.16); ${ }^{1} \mathrm{H}$ NMR: $\mathrm{CD}_{3} \mathrm{OD}$ (3.31), ${ }^{13} \mathrm{C}^{\mathrm{NMR}} \mathrm{CD} \mathrm{CD}_{3} \mathrm{OD}$ : (49.00)]. Peak multiplicities were recorded as follows: $\mathrm{s}=$ singlet, $\mathrm{d}=$ doublet, $\mathrm{t}=$ triplet, $\mathrm{q}=$ quartet, $\mathrm{m}=$ multiplet, br = broad singlet. Infrared (IR) spectra were recorded on a BRUKER Tensor-27 FourierTransform Infrared spectrometer. High resolution mass spectral (HRMS) data were obtained at the mass spectrometry service operated at Agilent 6540 Q-TOF spectrometer for electrospray ionization (ESI) and were reported as $(\mathrm{m} / \mathrm{z})$. X-ray diffraction was conducted using Bruker APEX DUO diffractometer with graphite-monochromated $\mathrm{CuK} \alpha$ radiation. Optical rotations were measured on a Jasco P-1020 polarimeter. Melting points were measured on a WRX-5A melting point apparatus. HPLC analysis was performed on an Agilent 1260 series system using Daicel Chiralpak AD-H and IC columns with $n$-hexane and $i$-PrOH as solvents. 


\section{Experimental Procedures}

\section{Optimization Tables}

Ir-Catalyzed Allylic Amination Reaction with $\mathrm{AcNH}_{2}{ }^{a}$

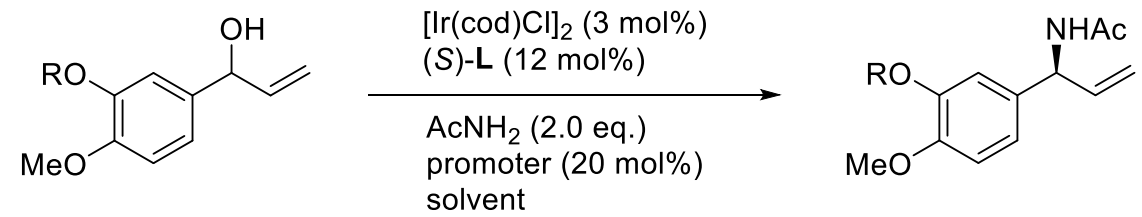

\section{General Procedure}

$[\operatorname{Ir}(\operatorname{cod}) \mathrm{Cl}]_{2}(2.0 \mathrm{mg}, 3 \mathrm{~mol} \%)$ and $(S)-\mathbf{L}(6.0 \mathrm{mg}, 12 \mathrm{~mol} \%)$ were dissolved in solvent under an atmosphere of nitrogen. The mixture was vigorously stirred for $15 \mathrm{~min}$ at room temperature. To the resulting solution were added sequentially allylic alcohol ( $0.1 \mathrm{mmol}, 1.0$ equiv), $\mathrm{AcNH}_{2}(12.0 \mathrm{mg}, 0.2$ mmol, 2.0 equiv), and promoter $(20 \mathrm{~mol} \%)$. The reaction was stirred at room temperature and monitored by TLC. The crude reaction mixture was quenched with sat. aq. $\mathrm{NaHCO}_{3}(5 \mathrm{~mL})$ and diluted with EtOAc $(10 \mathrm{~mL})$. The layers were separated and then the aqueous phase was extracted with EtOAc ( 2 x $10 \mathrm{~mL}$ ), combined organic layers dried over $\mathrm{Na}_{2} \mathrm{SO}_{4}$, filtered, and concentrated under reduced pressure. The crude residue was purified by silica gel flash chromatography to afford the desired product.

\begin{tabular}{|c|c|c|c|c|c|c|}
\hline entry & $\mathrm{R}$ & promoter & solvent & $\mathrm{T}$ & isolated yield (\%) & ee $(\%)^{b}$ \\
\hline $1^{c}$ & TBS & maleic acid & $\operatorname{DCE}(0.25 \mathrm{M})$ & $\mathrm{rt}$ & trace & - \\
\hline $2^{d}$ & TBS & $m-\mathrm{ClC}_{6} \mathrm{H}_{4} \mathrm{COOH}$ & $\operatorname{DCE}(0.25 \mathrm{M})$ & $50{ }^{\circ} \mathrm{C}$ & $<5$ & - \\
\hline 3 & TBS & $\mathrm{Zn}(\mathrm{OTf})_{2}$ & 1,4-dioxane $(0.5 \mathrm{M})$ & $\mathrm{rt}$ & 30 & 96 \\
\hline 4 & TBS & $\mathrm{Sc}(\mathrm{OTf})_{3}$ & 1,4-dioxane/DMF (5:1, 0.5 M) & $\mathrm{rt}$ & 36 & 98 \\
\hline 5 & TBS & $\mathrm{Sc}(\mathrm{OTf})_{3}$ & 1,4-dioxane/DMF (10:1, 0.25 M) & $\mathrm{rt}$ & 36 & $>99$ \\
\hline 6 & TBS & $\mathrm{Sc}(\mathrm{OTf})_{3}$ & 1,4-dioxane/DMF (10:1, $0.25 \mathrm{M})$ & $50{ }^{\circ} \mathrm{C}$ & 39 & $>99$ \\
\hline 7 & TBS & $\mathrm{Sc}(\mathrm{OTf})_{3}$ & 1,4-dioxane/DMF (10:1, 0.25 M) & $80{ }^{\circ} \mathrm{C}$ & 40 & 92 \\
\hline $8^{e}$ & TBS & $\mathrm{Sc}(\mathrm{OTf})_{3}$ & 1,4-dioxane/DMF (10:1, $0.25 \mathrm{M})$ & $50{ }^{\circ} \mathrm{C}$ & 33 & 95 \\
\hline
\end{tabular}




\begin{tabular}{ccccccc}
\hline 9 & $\mathrm{TBS}$ & $\mathrm{BF}_{3} \cdot \mathrm{Et}_{2} \mathrm{O}$ & 1,4-dioxane $(0.25 \mathrm{M})$ & $\mathrm{rt}$ & 78 & 95 \\
10 & $\mathrm{H}$ & $\mathrm{BF}_{3} \cdot \mathrm{Et}_{2} \mathrm{O}$ & 1,4-dioxane $(0.5 \mathrm{M})$ & $\mathrm{rt}$ & 90 & $>99$ \\
$11^{f}$ & $\mathrm{H}$ & $\mathrm{BF}_{3} \cdot \mathrm{Et}_{2} \mathrm{O}$ & $1,4-$ dioxane $(0.5 \mathrm{M})$ & $\mathrm{rt}$ & 93 & $>99$ \\
\hline
\end{tabular}

${ }^{a}$ Unless indicated otherwise, the reactions were performed on a $0.1 \mathrm{mmol} \mathrm{scale.}{ }^{b}$ Enantiomeric excess was determined by HPLC analysis ( $\mathrm{R}=\mathrm{TBS}$, Chiralpak IC; $\mathrm{R}=\mathrm{H}$, Chiralpak AD-H). ${ }^{c} 0.75$ equiv of promoter was used. ${ }^{d} 0.5$ equiv of promoter was used. ${ }^{e}$ Run on $0.5 \mathrm{mmol}$ scale. ${ }^{f}$ Run on $10.0 \mathrm{mmol}$ scale.

\section{Ir-Catalyzed Allylic Amination Reaction with $\mathrm{NH}_{3} \mathrm{SO}_{3}{ }^{a}$}<smiles>C=CC(O)c1ccc(OC)c(O)c1</smiles>

13

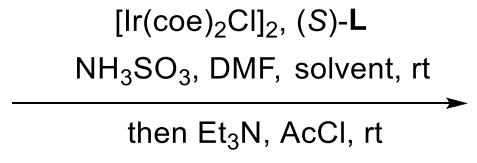

then $\mathrm{Et}_{3} \mathrm{~N}, \mathrm{AcCl}$, rt

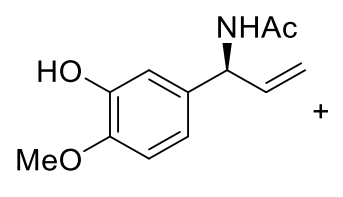

14<smiles>C=C[C@H](NC(C)(C)C)c1ccc(OC)c(OC(C)=O)c1</smiles>

$14^{\prime}$

\section{General Procedure}

$\left[\operatorname{Ir}(\text { coe })_{2} \mathrm{Cl}\right]_{2}(4.5 \mathrm{mg}, 2.5 \mathrm{~mol} \%),(S)-\mathbf{L}(10.0 \mathrm{mg}, 10 \mathrm{~mol} \%), \mathrm{NH}_{3} \mathrm{SO}_{3}(23 \mathrm{mg}, 0.24 \mathrm{mmol}, 1.2$ equiv) were placed in a round bottom flask with a magnetic stir bar. The reaction vessel was purged with nitrogen, DMF ( $80 \mu \mathrm{L}, 1.0 \mathrm{mmol}, 5.0$ equiv) was added followed by solvent $(0.33 \mathrm{M})$. After vigorous stirring for $10 \mathrm{~min}$, allylic alcohol 13 (36 mg, $0.2 \mathrm{mmol}, 1.0$ equiv) was added to the reaction mixture. The reaction was stirred at room temperature for $24 \mathrm{~h} . \mathrm{Et}_{3} \mathrm{~N}(0.11 \mathrm{~mL}, 0.8 \mathrm{mmol}, 4.0$ equiv. was then added followed by $\mathrm{AcCl}(28 \mu \mathrm{L}, 0.4 \mathrm{mmol}, 2.0$ equiv $)$ and the resulting mixture was stirred at room temperature for $4 \mathrm{~h}$. The reaction was then purified by flash chromatography (petroleum ether/EtOAc 1:1 to 1:2) to give the resulting products $\mathbf{1 4}$ and $\mathbf{1 4}^{\prime}$.

\begin{tabular}{ccccc}
\hline entry & solvent & yield of $\mathbf{1 4}(\%)^{b}$ & yield of $\mathbf{1 4}(\%)^{b}$ & ee of $\mathbf{1 4}(\%)^{c}$ \\
\hline 1 & DCE & $<5$ & 42 & 86 \\
2 & 1,4-dioxane & 36 & 30 & 87 \\
3 & 2 -MeTHF & 27 & 30 & 88
\end{tabular}

${ }^{a}$ Unless indicated otherwise, the reactions were performed on a 0.2 mmol scale. ${ }^{b}$ Isolated yields. ${ }^{c}$ Enantiomeric excess was determined by HPLC analysis (Chiralpak AD-H). 


\section{Suzuki Cross-Coupling Reaction ${ }^{a}$}

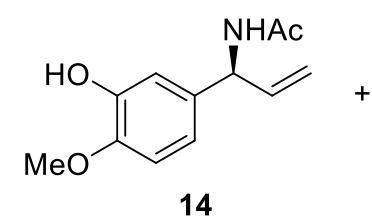

14

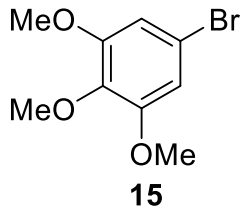

15

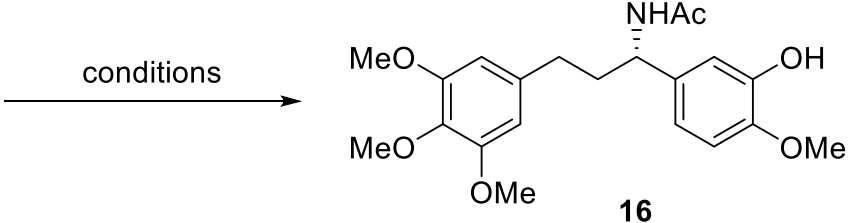

16

\begin{tabular}{|c|c|c|}
\hline entry & conditions & isolated yield (\%) \\
\hline 1 & $\begin{array}{c}\mathrm{BH}_{3} \cdot \mathrm{THF}(1.2 \text { eq. }), 0^{\circ} \mathrm{C} \text { to } \mathrm{rt} \text {, then } \mathrm{H}_{2} \mathrm{O} \text { (15.0 eq.), } 15 \text { (1.2 eq.), } \mathrm{Pd}\left(\mathrm{PPh}_{3}\right)_{4} \\
(10 \mathrm{~mol} \%), \mathrm{K}_{3} \mathrm{PO}_{4}(2.0 \text { eq. }) \text {, toluene, } 85^{\circ} \mathrm{C} \text {. }\end{array}$ & - \\
\hline 2 & $\begin{array}{c}\text { 9-BBN (2.0 eq.), } 50{ }^{\circ} \mathrm{C} \text {, then } \mathrm{H}_{2} \mathrm{O} \text { (15.0 eq.), } 15 \text { (1.2 eq.), } \mathrm{Pd}\left(\mathrm{PPh}_{3}\right)_{4}(10 \\
\text { mol\%), } \mathrm{K}_{3} \mathrm{PO}_{4}(2.0 \text { eq. }), 1 \text {, 4-dioxane, } 85^{\circ} \mathrm{C} .\end{array}$ & 23 \\
\hline 3 & $\begin{array}{l}\text { 9-BBN (4.0 eq.) } 50{ }^{\circ} \mathrm{C} \text {, then } \mathrm{H}_{2} \mathrm{O} \text { (15.0 eq.), } 15 \text { (1.2 eq.), } \mathrm{Pd}(\mathrm{dppf}) \mathrm{Cl}_{2}(3 \\
\text { mol\%), } \mathrm{AsPh}_{3}(3 \mathrm{~mol} \%), \mathrm{Cs}_{2} \mathrm{CO}_{3}\left(2.0 \text { eq.), DMF, } 50{ }^{\circ} \mathrm{C} .\right.\end{array}$ & 21 \\
\hline 4 & $\begin{array}{l}\text { 9-BBN (2.0 eq.) rt to } 50^{\circ} \mathrm{C} \text {, then } 15 \text { (2.0 eq.), } \mathrm{Pd}(\mathrm{dppf}) \mathrm{Cl}_{2}(3 \mathrm{~mol} \%), \mathrm{CsF} \\
\text { (3.0 eq.), } 60^{\circ} \mathrm{C} .\end{array}$ & 34 \\
\hline 5 & $\begin{array}{l}\text { 9-BBN (3.0 eq.) } 0{ }^{\circ} \mathrm{C} \text { to rt, then } \mathbf{1 5} \text { (2.0 eq.), } \mathrm{Pd}(\mathrm{dppf}) \mathrm{Cl}_{2}(3 \mathrm{~mol} \%), \mathrm{CsF} \\
\text { (3.0 eq.), } 60^{\circ} \mathrm{C} \text {. }\end{array}$ & 51 \\
\hline 6 & $\begin{array}{l}\text { 9-BBN (3.0 eq.), rt, then } \mathrm{H}_{2} \mathrm{O} \text { (15.0 eq.), } 15 \text { (2.0 eq.), } \mathrm{Pd}(\mathrm{dppf}) \mathrm{Cl}_{2}(3 \\
\text { mol\%), } \mathrm{CH}_{3} \mathrm{ONa}\left(3.0 \text { eq.), } 75^{\circ} \mathrm{C} .\right.\end{array}$ & 30 \\
\hline 7 & $\begin{array}{l}\text { 9-BBN (3.0 eq.), rt, then } \mathrm{H}_{2} \mathrm{O} \text { (15.0 eq.), } 15 \text { (3.0 eq.), } \mathrm{Pd}\left(\mathrm{PPh}_{3}\right)_{4}(10 \mathrm{~mol} \%) \text {, } \\
\qquad \mathrm{Ba}(\mathrm{OH})_{2} \cdot 8 \mathrm{H}_{2} \mathrm{O} \text { (3.0 eq.), reflux. }\end{array}$ & 28 \\
\hline 8 & $\begin{array}{l}\text { 9-BBN (3.0 eq.), rt, then } \mathrm{H}_{2} \mathrm{O} \text { (15.0 eq.), } 15 \text { (3.0 eq.), } \mathrm{Pd}\left(\mathrm{PPh}_{3}\right)_{4}(10 \mathrm{~mol} \%) \text {, } \\
\qquad \mathrm{NaOH} \text { (3.0 eq.), reflux. }\end{array}$ & 25 \\
\hline 9 & $\begin{array}{c}\text { 9-BBN (3.0 eq.), rt, then } \mathrm{H}_{2} \mathrm{O}\left(15.0 \text { eq.), } 15 \text { (2.0 eq.), } \mathrm{Pd}(\mathrm{OAc})_{2}(10 \mathrm{~mol} \%) \text {, }\right. \\
\text { Xphos (15 mol\%), KF (3.0 eq.), } 60^{\circ} \mathrm{C} .\end{array}$ & 42 \\
\hline 10 & $\begin{array}{l}\text { 9-BBN (3.0 eq.), rt, then } \mathrm{H}_{2} \mathrm{O}\left(15.0 \text { eq.), } 15 \text { (2.0 eq.), } \mathrm{Pd}(\mathrm{OAc})_{2}(10 \mathrm{~mol} \%) \text {, }\right. \\
\qquad \mathrm{PPh}_{3}(30 \mathrm{~mol} \%) \text {, DIPEA }(10.0 \text { eq }), 70^{\circ} \mathrm{C} \text {. }\end{array}$ & 10 \\
\hline 11 & $\begin{array}{l}\text { 9-BBN (3.0 eq.), rt, then } \mathrm{H}_{2} \mathrm{O} \text { (15.0 eq.), } 15 \text { (2.0 eq.), } \mathrm{Pd}(\mathrm{OAc})_{2}(10 \mathrm{~mol} \%) \text {, } \\
\qquad \mathrm{PCy}_{3}(30 \mathrm{~mol} \%), \operatorname{DIPEA}(10.0 \text { eq. }), 70^{\circ} \mathrm{C} .\end{array}$ & 30 \\
\hline
\end{tabular}


9-BBN (3.0 eq.), rt, then $\mathrm{H}_{2} \mathrm{O}$ (15.0 eq.), 15 (2.0 eq.), $\mathrm{Pd}(\mathrm{OAc})_{2}(10 \mathrm{~mol} \%)$,

$$
\text { Xphos (15 mol\%), DIPEA (10.0 eq.), } 70{ }^{\circ} \mathrm{C} \text {. }
$$

9-BBN (3.0 eq.), rt, then $\mathrm{H}_{2} \mathrm{O}$ (15.0 eq.), 15 (3.0 eq.), $\mathrm{Pd}\left(\mathrm{PPh}_{3}\right)_{4}(5 \mathrm{~mol} \%)$,

$$
\mathrm{Et}_{3} \mathrm{~N} \text { (10.0 eq.), reflux. }
$$

9-BBN (3.0 eq.), rt, then $\mathrm{H}_{2} \mathrm{O}$ (15.0 eq.), 15 (3.0 equiv), $\mathrm{Pd}\left(\mathrm{PPh}_{3}\right)_{4}(10$

$$
\text { mol\%), DIPEA (10.0 equiv), reflux. }
$$

9-BBN (3.0 eq.), $0{ }^{\circ} \mathrm{C}$ to rt, then $\mathrm{H}_{2} \mathrm{O}$ (15.0 eq.), 15 (1.2 eq.), $\mathrm{Pd}\left(\mathrm{PPh}_{3}\right)_{4}(10$

$$
\text { mol\%), } \mathrm{K}_{3} \mathrm{PO}_{4} \text { (2.0 eq.), DMF, reflux. }
$$

9-BBN (3.0 eq.), $0{ }^{\circ} \mathrm{C}$ to rt, then $\mathrm{H}_{2} \mathrm{O}$ (15.0 eq.), 15 (3.0 eq.), $\mathrm{Pd}\left(\mathrm{PPh}_{3}\right)_{4}(5$

mol\%) $\mathrm{K}_{3} \mathrm{PO}_{4}$ (3.0 eq.), DMF, reflux.

mol\%), $\mathrm{K}_{3} \mathrm{PO}_{4}$ (3.0 eq.), DMF, reflux.

${ }^{a}$ Unless indicated otherwise, the reactions were performed on a $0.1-0.2$ mmol scale. ${ }^{b}$ Run on $2.0 \mathrm{mmol}$ scale. ${ }^{c}$ Run on $9.28 \mathrm{mmol}$ scale.

\section{Intermolecular Heck-Hydrogenation Reaction ${ }^{3}$}<smiles>C=CC(C)c1ccc(OC)c(O)c1</smiles>

14<smiles>COc1cc(P)cc(OC)c1OC</smiles>

15<smiles>COc1ccc(CCCc2cc(OC)c(OC)c(OC)c2)cc1O</smiles>

16

Note: the reaction was performed on $0.2 \mathrm{mmol}$ scale, and the isolated yield of $\mathbf{1 6}$ was improved while the enantiomeric excess significantly decreased. 


\section{Intramolecular Oxidative Coupling Reaction of Compound $16^{a}$}
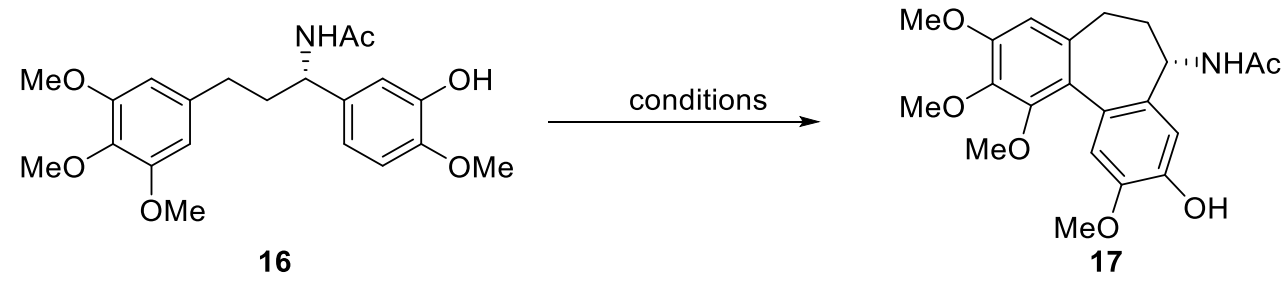

\begin{tabular}{|c|c|c|}
\hline entry & conditions & yield $(\%)^{b}$ \\
\hline 1 & $\mathrm{PhI}(\mathrm{OAc})_{2}(1.0$ eq. $), \mathrm{AcOH}$ (2.0 eq.), HFIP, rt & trace \\
\hline 2 & PIFA (1.0 eq.), $\mathrm{MeOH}$, rt, then evaporation, $\mathrm{BF}_{3} \mathrm{Et}_{2} \mathrm{O}$ (2.0 eq.), DCM, $-40{ }^{\circ} \mathrm{C}$. & 53 \\
\hline 3 & PIFA (1.0 eq.), $\mathrm{BF}_{3} \cdot \mathrm{Et}_{2} \mathrm{O}$ (2.0 eq.). TFA/TFAA/DCM $(4: 1: 5),-40^{\circ} \mathrm{C}$. & 54 \\
\hline 4 & PIFA (1.0 eq. $), \mathrm{MeOH}, \mathrm{rt}$, then evaporation, $\mathrm{Sc}(\mathrm{OTf})_{3}(0.2$ eq. $), \mathrm{DCM}, 0{ }^{\circ} \mathrm{C}$. & 55 \\
\hline 5 & PIFA (1.0 eq.), $\mathrm{MsOH}$ (2.0 eq.), THF, rt, then $\mathrm{Na}_{2} \mathrm{~S}_{2} \mathrm{O}_{3}\left(0.1\right.$ eq.), $80{ }^{\circ} \mathrm{C}$. & trace \\
\hline 6 & PIFA (1.0 eq.), $\mathrm{BF}_{3} \cdot \mathrm{Et}_{2} \mathrm{O}$ (2.0 eq.), THF/MeOH (5:1), rt & 35 \\
\hline 7 & PIFA (1.0 eq.), $\mathrm{BF}_{3} \cdot \mathrm{Et}_{2} \mathrm{O}$ (2.0 eq.), DCM/MeOH (5:1), rt.. & 59 \\
\hline 8 & $(t-\mathrm{Bu}-\mathrm{O})_{2}(3.0$ eq. $), \mathrm{FeCl}_{3}(0.15$ eq. $), \mathrm{HFIP}, \mathrm{rt}$. & - \\
\hline 9 & $\mathrm{H}_{5} \mathrm{IO}_{6}$ (0.5 eq.), DMF (2.5 eq.), HFIP, rt. & - \\
\hline 10 & PIFA (1.0 eq.), $\mathrm{BF}_{3} \cdot \mathrm{Et}_{2} \mathrm{O}$ (2.0 eq.), $\mathrm{HFIP}, 0^{\circ} \mathrm{C}$ to rt. & trace \\
\hline 11 & $\mathrm{~K}_{2} \mathrm{~S}_{2} \mathrm{O}_{8}$ (2.0 eq.), TBAHS ( 0.1 eq.), TFA, rt. & $<5$ \\
\hline 12 & PIFA (1.0 eq.), TFA/DCM (2:1), rt. & 50 \\
\hline 13 & PIFA (1.0 eq.), AcOH/DCM (2:1), rt. & 45 \\
\hline 14 & PIFA (1.0 eq.), MeOH/TFA (5:1), rt. & 42 \\
\hline 15 & PIFA (1.0 eq.), $\mathrm{MeOH}$, rt; no evaporation, then $\mathrm{BF}_{3} \mathrm{Et}_{2} \mathrm{O}$ (3.0 eq.), DCM, rt & 78 \\
\hline 15 & $\mathrm{PhI}(\mathrm{OAc})_{2}(1.0$ eq. $), \mathrm{MeOH}$, rt; no evaporation, then $\mathrm{BF}_{3} \mathrm{Et}_{2} \mathrm{O}$ (3.0 eq.), DCM, rt & 82 \\
\hline $16^{c}$ & $\mathrm{PhI}(\mathrm{OAc})_{2}(1.0$ eq. $), \mathrm{MeOH}$, rt; no evaporation, then $\mathrm{BF}_{3} \mathrm{Et}_{2} \mathrm{O}$ (3.0 eq.), DCM, rt & 80 \\
\hline
\end{tabular}

${ }^{a}$ Unless indicated otherwise, the reactions were performed on a $0.05 \mathrm{mmol}$ scale. ${ }^{b}$ Isolated yields. ${ }^{c}$ Run on $1.3 \mathrm{mmol}$ scale. 


\section{Cyclopropane Ring-Cleavage Reaction of Compound $19^{a}$}
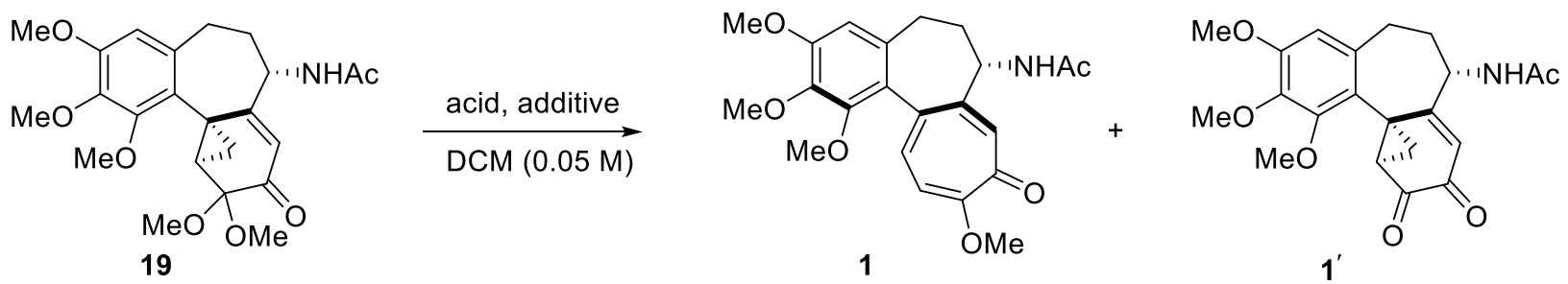

1

\begin{tabular}{cccccccc}
\hline entry & acid & eq. of acid & additive & $\mathrm{T}\left({ }^{\circ} \mathrm{C}\right)$ & $t(\mathrm{~h})$ & yield $(\%)^{b}$ & $\mathbf{1}^{\prime \mathbf{1}^{c}}$ \\
\hline 1 & $\mathrm{TFA}$ & 10.0 & - & $\mathrm{rt}$ & 1 & 80 & $1: 1$ \\
2 & $\mathrm{BF}_{3} \cdot \mathrm{Et}_{2} \mathrm{O}$ & 1.1 & - & $\mathrm{rt}$ & 0.25 & 84 & $1: 3.3$ \\
3 & $p-\mathrm{TSA}^{2} \cdot \mathrm{H}_{2} \mathrm{O}$ & 1.1 & - & $\mathrm{rt}$ & 1 & 82 & $1: 6.3$ \\
4 & $\mathrm{BF}_{3} \cdot \mathrm{Et}_{2} \mathrm{O}$ & 2.2 & $4 \AA$ molecular sieves & $\mathrm{rt}$ & 18 & 65 & $1: 2.7$ \\
5 & $\mathrm{TFA}$ & 10.0 & $4 \AA$ molecular sieves & $\mathrm{rt}$ & 12 & 78 & $3.6: 1$ \\
6 & $\mathrm{TFA}$ & 10.0 & $4 \AA$ molecular sieves & 40 & 2 & 88 & $8: 1$ \\
\hline
\end{tabular}

${ }^{a}$ Unless indicated otherwise, the reactions were performed on a $0.025 \mathrm{mmol}$ scale. ${ }^{b}$ Yields of $\mathbf{1}$ plus $\mathbf{1}^{\prime}$. ${ }^{c}$ Determined by ${ }^{1} \mathrm{H}$ NMR of crude reaction mixture. 


\section{Synthetic Procedures and Characterization Data}

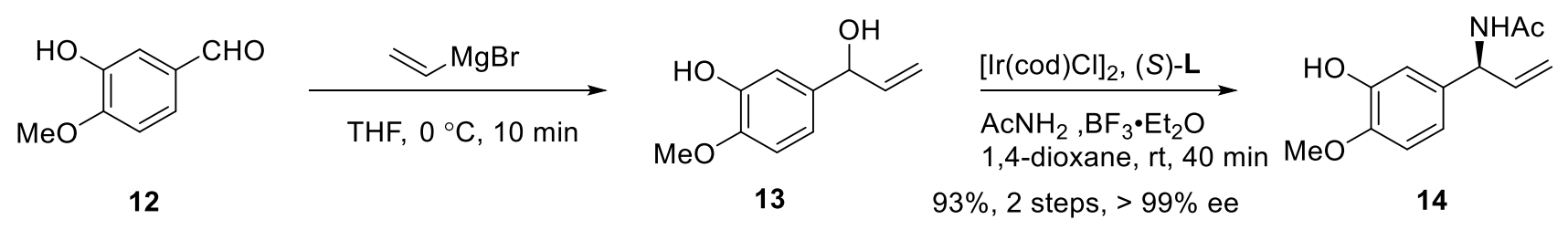

To a solution of isovanillin $12(1.52 \mathrm{~g}, 10.0 \mathrm{mmol}, 1.0$ equiv) in THF (150 mL) was added a solution of vinylmagnesium bromide $\left(40 \mathrm{~mL}, 1.0 \mathrm{M}\right.$ in THF, $40.0 \mathrm{mmol}, 4.0$ equiv) at $0{ }^{\circ} \mathrm{C}$ in an ice bath. The reaction mixture was stirred for $10 \mathrm{~min}$ at $0{ }^{\circ} \mathrm{C}$ and then quenched with sat. aq. $\mathrm{NH}_{4} \mathrm{Cl}(30$ $\mathrm{mL})$, The layers were separated and then the aqueous phase was extracted with EtOAc $(2 \times 100 \mathrm{~mL})$, combined organic layers were washed with brine, dried over $\mathrm{Na}_{2} \mathrm{SO}_{4}$, filtered, and concentrated under reduced pressure. The crude residue $\mathbf{1 3}$ was used in the subsequent step without further purification.

A flame-dried $100 \mathrm{~mL}$ round-bottom flask equipped with a stir bar was charged with $[\mathrm{Ir}(\mathrm{cod}) \mathrm{Cl}]_{2}$ (201 mg, $0.3 \mathrm{mmol}, 3 \mathrm{~mol} \%)$, (S)-L (611 mg, $1.2 \mathrm{mmol}, 12 \mathrm{~mol} \%)$ and 1,4-dioxane (15 mL) under an atmosphere of nitrogen. After vigorous stirring for $10 \mathrm{~min}$ at room temperature, a solution of the crude product 13 in $5 \mathrm{~mL}$ 1,4-dioxane obtained in the prior transformation, $\mathrm{AcNH}_{2}(1.18 \mathrm{~g}, 20.0 \mathrm{mmol}, 2.0$ equiv), and promoter $\mathrm{BF}_{3} \cdot \mathrm{Et}_{2} \mathrm{O}(252 \mu \mathrm{L}, 2.0 \mathrm{mmol}, 20 \mathrm{~mol} \%)$ were added sequentially to the resulting solution. The reaction mixture was stirred for $30 \mathrm{~min}$ at room temperature and then quenched with sat. aq. $\mathrm{NaHCO}_{3}(5 \mathrm{~mL})$. The layers were separated and then the aqueous phase was extracted with EtOAc $(2 \times 50 \mathrm{~mL})$, combined organic layers were washed with brine, dried over $\mathrm{Na}_{2} \mathrm{SO}_{4}$, filtered, and concentrated under reduced pressure. The crude residue was purified by silica gel flash chromatography (petroleum ether/EtOAc 1:1 to 1:2) to afford the desired product 14 (2.05 $\mathrm{g}, 93 \%$ yield, 2 steps) as a yellow oil.

\section{Data for compound $\mathbf{1 3}$}

Compound 13 was isolated for characterization purposes by purifying a small amount of crude by column chromatography (petroleum ether/EtOAc 2:1).

$\mathbf{R}_{f}$ : 0.31 (petroleum ether/EtOAc 2:1).

Physical state: white solid

m.p.: $93-95^{\circ} \mathrm{C}$. 
${ }^{1} \mathbf{H}$ NMR $\left(400 \mathrm{MHz}, \mathrm{CDCl}_{3}\right) \delta 6.95(\mathrm{~s}, 1 \mathrm{H}), 6.85(\mathrm{q}, J=8.3 \mathrm{~Hz}, 2 \mathrm{H}), 6.03(\mathrm{ddd}, J=16.8,10.3,5.9 \mathrm{~Hz}$, 1H), $5.65(\mathrm{~s}, 1 \mathrm{H}), 5.34(\mathrm{~d}, J=17.1 \mathrm{~Hz}, 1 \mathrm{H}), 5.18(\mathrm{~d}, J=10.3 \mathrm{~Hz}, 1 \mathrm{H}), 5.12(\mathrm{~d}, J=5.3 \mathrm{~Hz}, 1 \mathrm{H}), 3.89$ $(\mathrm{s}, 3 \mathrm{H}), 1.92(\mathrm{~s}, 1 \mathrm{H})$.

${ }^{13}$ C NMR (100 MHz, $\left.\mathrm{CDCl}_{3}\right) \delta 146.3,145.8,140.3,136.2,118.1,115.0,112.9,110.7,75.1,56.1$.

IR (KBr): 3447, 3192, 2897, 1615, 1599, 1537, 1437, 1386, 1279, 1255, 1181, 1159, 1026, 976, 869, $809,763,488 \mathrm{~cm}^{-1}$.

HRMS (ESI): calc. for $\mathrm{C}_{10} \mathrm{H}_{11} \mathrm{O}_{3}[\mathrm{M}-\mathrm{H}]^{-}: 179.0714$, found: 179.0714.

Data for compound 14

$\mathbf{R}_{\boldsymbol{f}}: 0.63$ (EtOAc).

Physical state: yellow oil

${ }^{1}$ H NMR $\left(400 \mathrm{MHz}, \mathrm{CDCl}_{3}\right) \delta 6.86(\mathrm{~s}, 1 \mathrm{H}), 6.83-6.76(\mathrm{~m}, 2 \mathrm{H}), 5.97(\mathrm{ddd}, J=15.9,10.4,5.1 \mathrm{~Hz}$, 1H), $5.80-5.67(\mathrm{~m}, 1 \mathrm{H}), 5.74(\mathrm{~s}, 1 \mathrm{H}), 5.60-5.48(\mathrm{~m}, 1 \mathrm{H}), 5.29-5.15(\mathrm{~m}, 2 \mathrm{H}), 3.88(\mathrm{~s}, 3 \mathrm{H}), 2.02$ $(\mathrm{s}, 3 \mathrm{H})$.

${ }^{13}$ C NMR (100 MHz, $\left.\mathrm{CDCl}_{3}\right) \delta 169.3,146.3,146.0,137.4,133.9,119.1,115.6,113.6,110.9,56.1$, $54.8,23.5$.

IR (KBr): 3281, 3084, 2841, 1651, 1636, 1511, 1441, 1276, 1133, 1028, 812, 762, $610 \mathrm{~cm}^{-1}$.

HRMS (ESI): calc. for $\mathrm{C}_{12} \mathrm{H}_{14} \mathrm{NO}_{3}[\mathrm{M}-\mathrm{H}]^{-}:$220.0979, found: 220.0979 .

Optical rotation: $[\alpha]_{\mathrm{D}}^{23.0}=-108.17^{\circ}\left(c 0.82, \mathrm{CHCl}_{3}\right)$.

HPLC analysis indicated that the enantiomeric excess of the product was $>99 \%$ [Diacel Chiralpak $\mathrm{AD}-\mathrm{H}$; hexanes $/ i-\mathrm{PrOH}=80 / 20$; flow rate $=1.0 \mathrm{~mL} / \mathrm{min} ; 25^{\circ} \mathrm{C}$; detection wavelength $=210 \mathrm{~nm} ; \mathrm{t}_{1}=$ $10.128 \mathrm{~min} ; \mathrm{t}_{2}=11.126 \mathrm{~min}$ (major)].

Racemicte compound $\mathbf{1 4}$ was acquired by using the above procedure with racemic ligand ( $\mathrm{rac})$-L instead of $(S)-\mathbf{L}$.

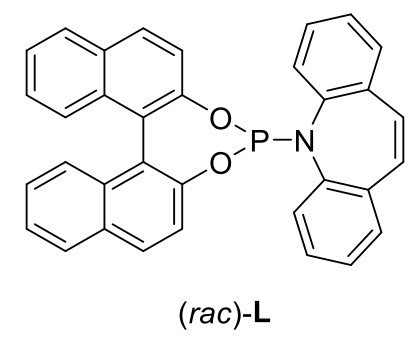


<smiles>C=C[C@H](N)c1ccc(OC)c(O)c1</smiles>

$(-)-14$

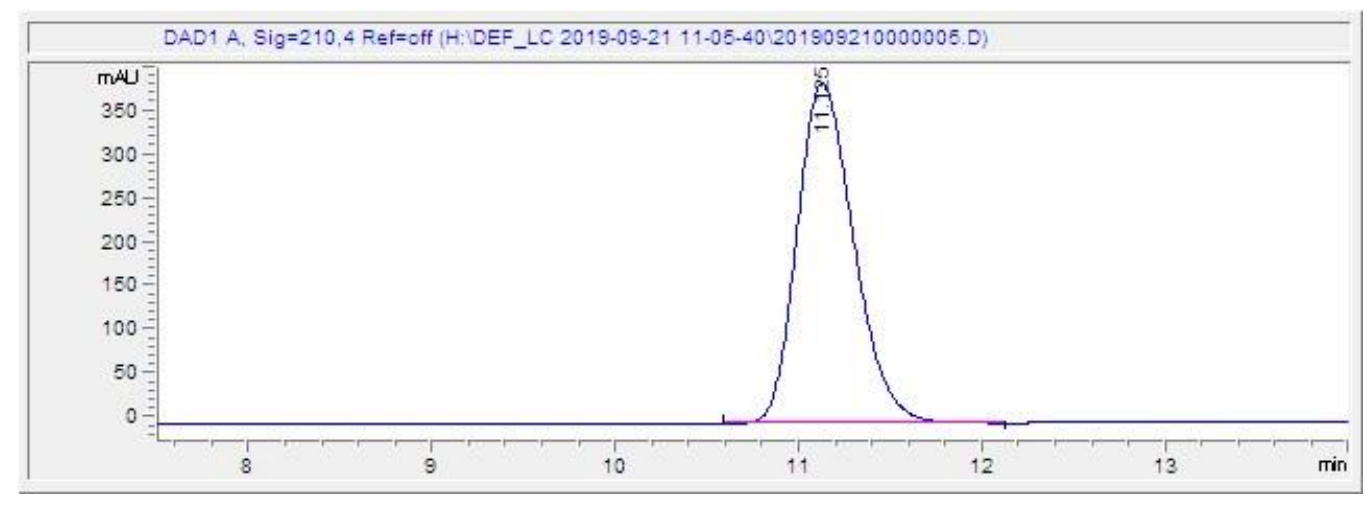

\begin{tabular}{l|c|c|c|c|c|c|} 
\# & \multicolumn{1}{c}{ Time } & \multicolumn{1}{c}{ Area } & \multicolumn{1}{c}{ Height } & Width & \multicolumn{1}{c}{ Area\% } & Symmetry \\
\hline 1 & 11.125 & 8353 & 388 & 0.3352 & 100.000 & 0.752 \\
\hline
\end{tabular}

rac-14

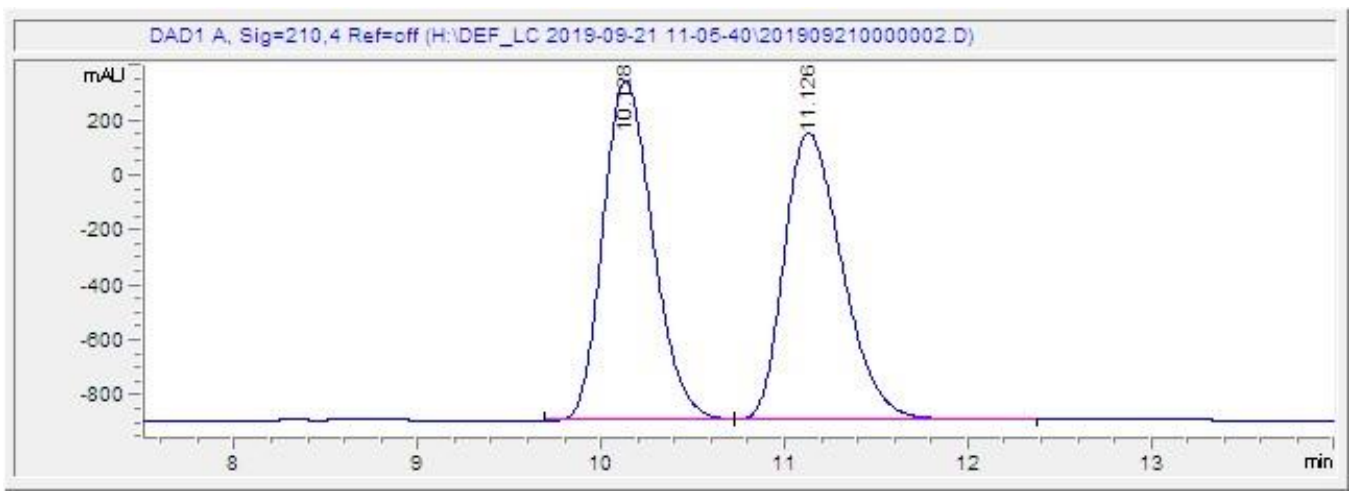

\begin{tabular}{|c|c|c|c|c|c|c|}
\hline$\#$ & Time & Area & Height & Width & Area\% & Symmetry \\
\hline 1 & 10.128 & 23333.8 & 1231.7 & 0.295 & 50.414 & 0.786 \\
\hline 2 & 11.126 & 22950.3 & 1043 & 0.3447 & 49.586 & 0.681 \\
\hline
\end{tabular}


<smiles>C=C[C@H](N)c1ccc(OC)c(O)c1</smiles>

14

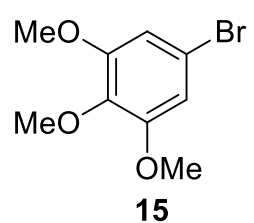

15

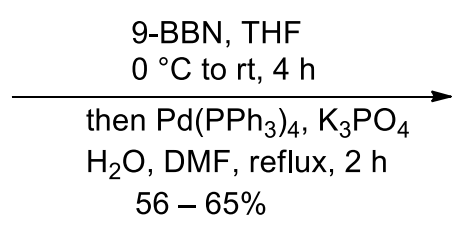

$56-65 \%$

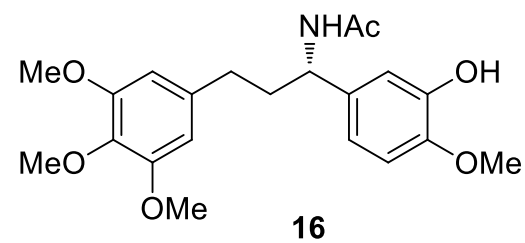

16

To a solution of compound 14 (2.05 g, $9.28 \mathrm{mmol}, 1.0$ equiv) in THF (5 mL) was added a solution of 9-BBN (56 mL, $0.5 \mathrm{M}$ in THF, $27.8 \mathrm{mmol}, 3.0$ equiv) at $0{ }^{\circ} \mathrm{C}$ in an ice bath. The reaction mixture was warmed up slowly to room temperature and stirred for $4 \mathrm{~h}$. And then the reaction was cooled down to $0{ }^{\circ} \mathrm{C}$ in an ice bath, quenched with $\mathrm{H}_{2} \mathrm{O}(2.5 \mathrm{~mL}, 139 \mathrm{mmol}, 15.0$ equiv $)$ and vigorously stirred for another $15 \mathrm{~min}$. To the resulting mixture were added successively 15 (6.8 g, $27.8 \mathrm{mmol}, 3.0$ equiv), $\mathrm{Pd}\left(\mathrm{PPh}_{3}\right)_{4}(535 \mathrm{mg}, 0.5 \mathrm{mmol}, 5 \mathrm{~mol} \%)$, powdered $\mathrm{K}_{3} \mathrm{PO}_{4}(5.9 \mathrm{~g}, 27.8 \mathrm{mmol}, 3.0$ equiv) and DMF (9 $\mathrm{mL}, 1.0 \mathrm{M})$. The resulting solution was degassed under a stream of nitrogen for $0.5 \mathrm{~h}$, and then heated to reflux in an oil bath and stirred for $2 \mathrm{~h}$. After the mixture was cooled down to room temperature, quenched with $\mathrm{H}_{2} \mathrm{O}(30 \mathrm{~mL})$ and diluted with EtOAc $(100 \mathrm{~mL})$. The layers were separated and the aqueous phase was extracted with EtOAc $(2 \times 100 \mathrm{~mL})$, combined organic layers were washed with brine $(3 \times 10 \mathrm{~mL})$, dried over $\mathrm{Na}_{2} \mathrm{SO}_{4}$, filtered, and concentrated under reduced pressure. The crude residue was purified by silica gel flash chromatography (petroleum ether/EtOAc 1:1 to 1:2) to afford the desired product $16(2.02 \mathrm{~g}, 56 \%$ yield $)$ as a yellow oil.

Data for compound 16

$\mathbf{R}_{\boldsymbol{f}}: 0.58$ (EtOAc).

Physical state: yellow oil.

${ }^{1}$ H NMR $\left(400 \mathrm{MHz}, \mathrm{CDCl}_{3}\right) \delta 6.87(\mathrm{~s}, 1 \mathrm{H}), 6.83-6.75(\mathrm{~m}, 2 \mathrm{H}), 6.35(\mathrm{~s}, 2 \mathrm{H}), 5.92(\mathrm{~s}, 1 \mathrm{H}), 5.79(\mathrm{~d}, J$ $=8.2 \mathrm{~Hz}, 1 \mathrm{H}), 4.91(\mathrm{q}, J=7.6 \mathrm{~Hz}, 1 \mathrm{H}), 3.86(\mathrm{~s}, 3 \mathrm{H}), 3.82(\mathrm{~s}, 6 \mathrm{H}), 3.80(\mathrm{~s}, 3 \mathrm{H}), 2.60-2.41(\mathrm{~m}, 2 \mathrm{H})$, $2.22-2.08(\mathrm{~m}, 1 \mathrm{H}), 2.07-1.94(\mathrm{~m}, 1 \mathrm{H}), 1.95(\mathrm{~s}, 3 \mathrm{H})$.

${ }^{13}$ C NMR $\left(100 \mathrm{MHz}, \mathrm{CDCl}_{3}\right) \delta 169.4,153.2,146.2,146.1,137.3,136.2,135.1,118.8,112.7,110.9$, $105.3,60.9,56.2,56.1,53.1,37.6,33.1,23.6$.

IR (KBr): 3355, 2939, 2839, 1652, 1591, 1509, 1459, 1275, 1240, 1127, 1008, 811, 763, $598 \mathrm{~cm}^{-1}$. HRMS (ESI): calc. for $\mathrm{C}_{21} \mathrm{H}_{27} \mathrm{NO}_{6} \mathrm{Na}[\mathrm{M}+\mathrm{Na}]^{+}:$412.1731, found: 412.1730 .

Optical rotation: $[\alpha]_{\mathrm{D}}{ }^{20.8}=-52.52^{\circ}\left(c 0.46, \mathrm{CHCl}_{3}\right)$. 
<smiles>COc1ccc([C@@H](CCc2ccccc2)NCCc2cc(OC)c(OC)c(OC)c2)cc1O</smiles>

$\mathrm{Phl}(\mathrm{OAc})_{2}, \mathrm{MeOH}, \mathrm{rt}, 5 \mathrm{~min}$ then $\mathrm{BF}_{3} \cdot \mathrm{Et}_{2} \mathrm{O}$ DCM, rt, 30 min<smiles>CCNCCN[C@@H]1CCc2cc(OC)c(OC)c(OC)c2-c2cc(OC)c(O)cc21</smiles>

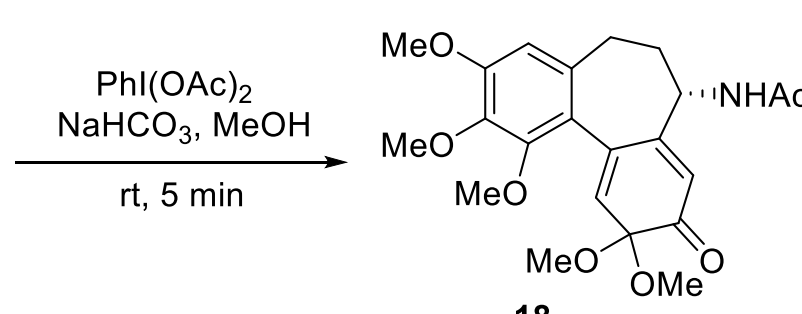

18

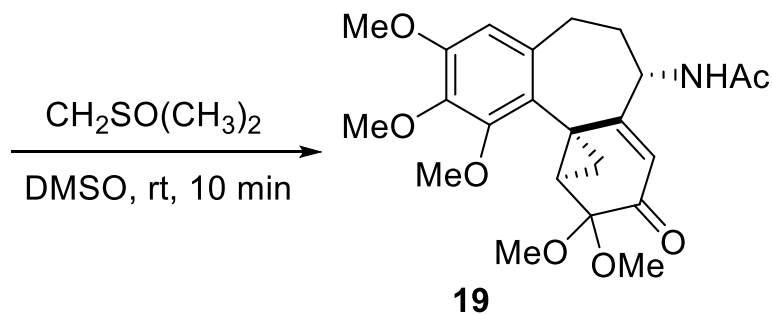

19

0.3- $5.2 \mathrm{mmol}$ scale

$51-60 \%$ over 4 steps

To a solution of compound 16 (2.02 g, $5.2 \mathrm{mmol}, 1.0$ equiv) in $\mathrm{MeOH}(10 \mathrm{~mL}, 0.5 \mathrm{M})$ was added solid $\mathrm{PhI}(\mathrm{OAc})_{2}(1.67 \mathrm{~g}, 5.2 \mathrm{mmol}, 1.0$ equiv) at room temperature. After stirring for $5 \mathrm{~min}$, this mixture was transferred slowly to a solution of $\mathrm{BF}_{3} \cdot \mathrm{Et}_{2} \mathrm{O}(2.0 \mathrm{~mL}, 15.6 \mathrm{mmol}, 3.0$ equiv) in DCM (520 $\mathrm{mL}, 0.01 \mathrm{M}$ ) via the syringe over $30 \mathrm{~min}$. The reaction mixture was stirred for $10 \mathrm{~min}$ and quenched with sat. aq. $\mathrm{NaHCO}_{3}(30 \mathrm{~mL})$. The layers were separated and the aqueous phase was extracted with $\mathrm{CHCl}_{3}(3 \times 50 \mathrm{~mL})$, combined organic layers were washed with brine, dried over $\mathrm{Na}_{2} \mathrm{SO}_{4}$, filtered, and concentrated under reduced pressure. The crude residue 17 was used in the subsequent step without further purification.

The above crude reside 17 was dissolved in $\mathrm{MeOH}(26 \mathrm{~mL}, 0.2 \mathrm{M})$, and powered $\mathrm{NaHCO}_{3}(872$ $\mathrm{mg}, 10.4 \mathrm{mmol}, 2.0$ equiv), $\mathrm{PhI}(\mathrm{OAc})_{2}(1.67 \mathrm{~g}, 5.2 \mathrm{mmol}, 1.0$ equiv) were added at room temperature. After stirring for $5 \mathrm{~min}$, the reaction mixture was quenched with sat. aq. $\mathrm{Na}_{2} \mathrm{~S}_{2} \mathrm{O}_{3}(20 \mathrm{~mL})$ and diluted with $\mathrm{Et}_{2} \mathrm{O} / \mathrm{EtOAc}(1: 1,80 \mathrm{~mL})$, the layers were separated and the aqueous phase was extracted with $\mathrm{Et}_{2} \mathrm{O} /$ EtOAc $(1: 1,4 \times 80 \mathrm{~mL})$, combined organic layers were washed with brine, dried over $\mathrm{Na}_{2} \mathrm{SO}_{4}$, filtered, and concentrated under reduced pressure. The crude residue $\mathbf{1 8}$ was used in the subsequent step without further purification. 
To a solution of trimethyl sulfoxonium iodide $(2.2 \mathrm{~g}, 10 \mathrm{mmol})$ in DMSO $(20 \mathrm{~mL})$ was added slowly $\mathrm{NaH}$ (440 mg, 60\% dispersion in mineral oil, $11 \mathrm{mmol}$ ) at room temperature, and the resulting mixture was stirred at room temperature until gas evolution had ceased. An aliquot ( $10.5 \mathrm{~mL}, 1.0$ equiv) of this solution was added dropwise via the syringe to a stirred solution of the above crude residue $\mathbf{1 8}$ in DMSO (13 mL, 0.4 M). The reaction mixture was stirred at room temperature for $10 \mathrm{~min}$, and then poured into water and extracted with $\mathrm{Et}_{2} \mathrm{O} / \mathrm{EtOAc}(1: 1,4 \times 80 \mathrm{~mL})$, The combined organic phase was washed with brine, dried over $\mathrm{Na}_{2} \mathrm{SO}_{4}$, filtered and concentrated under reduced pressure. The crude residue 19 was used in the subsequent step without further purification.

A solution of the above crude residue 19 in DCM $(100 \mathrm{~mL}, 0.05 \mathrm{M})$ was treated with $4 \AA$ molecular sieves $(6.0 \mathrm{~g})$ at room temperature. After stirring for $15 \mathrm{~min}$, TFA (3.8 mL, $52 \mathrm{mmol}, 10.0$ equiv) was added dropwise to the resulting suspension. The reaction was heated to $40{ }^{\circ} \mathrm{C}$ in an oil bath and stirred for $2 \mathrm{~h}$. And then the mixture was cooled to room temperature, quenched with sat. aq. $\mathrm{NaHCO}_{3}(50$ $\mathrm{mL})$, the layers were separated and the aqueous phase was extracted with $\mathrm{CHCl}_{3}(4 \times 50 \mathrm{~mL})$, combined organic layers were washed with brine, dried over $\mathrm{Na}_{2} \mathrm{SO}_{4}$, filtered, and concentrated under reduced pressure. The crude residue was purified by silica gel flash chromatography (petroleum ether/acetone 1:1 to $1: 4)$ to afford colchicine 1 (1.1 g, 53\% yield, 4 steps) as a dark yellow solid.

\section{Data for compound $17^{4}$}

Compound 17 was isolated for characterization purposes by purifying a small amount of crude by column chromatography (petroleum ether/EtOAc 1:1 to 1:4). A sample of compound $\mathbf{1 7}$ for X-ray analysis was recrystallized from $n$-hexane/ acetone (2:1) via slow evaporation to afford colorless needles.

$\mathbf{R}_{\boldsymbol{f}}: 0.43$ (EtOAc).

Physical state: light yellow solid.

m.p.: $130-133{ }^{\circ} \mathrm{C}$.

${ }^{1} \mathrm{H}$ NMR (600 MHz, CD $\left.{ }_{3} \mathrm{OD}\right): \delta 7.00(\mathrm{~s}, 1 \mathrm{H}), 6.80(\mathrm{~s}, 1 \mathrm{H}), 6.72(\mathrm{~s}, 1 \mathrm{H}), 4.58(\mathrm{dd}, J=12.4,6.0 \mathrm{~Hz}$, 1H), 3.88 (s, 3H), $3.87(\mathrm{~s}, 3 \mathrm{H}), 3.85(\mathrm{~s}, 3 \mathrm{H}), 3.53(\mathrm{~s}, 3 \mathrm{H}), 2.56-2.44(\mathrm{~m}, 1 \mathrm{H}), 2.31-2.21(\mathrm{~m}, 2 \mathrm{H})$, $2.00(\mathrm{~s}, 3 \mathrm{H}), 1.94-1.86(\mathrm{~m}, 1 \mathrm{H})$.

${ }^{13} \mathrm{C}$ NMR (150 MHz, $\left.\mathrm{CD}_{3} \mathrm{OD}\right): \delta 172.4,153.8,152.0,147.3,147.0,142.4,136.9,134.0,126.7,126.6$, $114.8,111.2,109.1,61.6,61.3,56.7,56.6,50.0,40.3,31.6,22.6$. 
IR (ATR): 3296, 2936, 2837, 1655, 1589, 1510, 1490, 1406, 1294, 1240, 1127, 1106, 1050, $780 \mathrm{~cm}^{-1}$.

HRMS (ESI): calc. for $\mathrm{C}_{21} \mathrm{H}_{25} \mathrm{NO}_{6} \mathrm{Na}[\mathrm{M}+\mathrm{Na}]^{+}: 410.1574$, found: 410.1573 .

Optical rotation: $[\alpha]_{\mathrm{D}}^{20.8}=-45.32^{\circ}\left(c 0.75, \mathrm{CHCl}_{3}\right)$.

Data for compound 18

Compound 18 was isolated for characterization purposes by purifying a small amount of crude by column chromatography (petroleum ether/EtOAc 1:1 to 1:2).

$\mathbf{R}_{f}: 0.52$ (EtOAc).

Physical state: yellow viscous oil.

${ }^{1} \mathbf{H}$ NMR $\left(400 \mathrm{MHz}, \mathrm{CDCl}_{3}\right) \delta 6.49(\mathrm{~s}, 1 \mathrm{H}), 6.43(\mathrm{~s}, 1 \mathrm{H}), 6.11(\mathrm{~d}, J=7.8 \mathrm{~Hz}, 1 \mathrm{H}), 6.01(\mathrm{~s}, 1 \mathrm{H}), 4.57$ - $4.44(\mathrm{~m}, 1 \mathrm{H}), 3.89(\mathrm{~s}, 3 \mathrm{H}), 3.87(\mathrm{~s}, 3 \mathrm{H}), 3.76(\mathrm{~s}, 3 \mathrm{H}), 3.43(\mathrm{~s}, 3 \mathrm{H}), 3.41(\mathrm{~s}, 3 \mathrm{H}), 2.72$ (td, $J=13.5$, $6.9 \mathrm{~Hz}, 1 \mathrm{H}), 2.59$ (dd, $J=13.8,6.2 \mathrm{~Hz}, 1 \mathrm{H}), 2.18(\mathrm{tt}, J=13.2,6.9 \mathrm{~Hz}, 1 \mathrm{H}), 1.98(\mathrm{~s}, 3 \mathrm{H}), 1.73-1.62$ $(\mathrm{m}, 1 \mathrm{H})$.

${ }^{13}$ C NMR (100 MHz, $\left.\mathrm{CDCl}_{3}\right) \delta$ 195.5, 169.4, 156.9, 153.6, 152.1, 141.7, 135.7, 133.8, 132.8, 121.9, 118.6, 107.8, 91.9, 61.7, 61.4, 56.2, 50.7, 50.3, 49.7, 34.0, 30.3, 23.2.

IR (KBr): 3371, 2941, 2836, 1672, 1596, 1538, 1490, 1460, 1324, 1105, 869, 753, $598 \mathrm{~cm}^{-1}$.

HRMS (ESI): calc. for $\mathrm{C}_{22} \mathrm{H}_{27} \mathrm{NO}_{7} \mathrm{Na}[\mathrm{M}+\mathrm{Na}]^{+}: 440.1680$, found: 440.1683 .

Optical rotation: $[\alpha]_{\mathrm{D}}{ }^{21.2}=36.74^{\circ}\left(c 0.55, \mathrm{CHCl}_{3}\right)$.

Data for compound 19

Compound 19 was isolated for characterization purposes by purifying a small amount of crude by column chromatography (petroleum ether/EtOAc 1:1 to 1:5).

$\mathbf{R}_{f}: 0.32$ (EtOAc).

Physical state: light yellow oil.

${ }^{1}$ H NMR $\left(600 \mathrm{MHz}, \mathrm{CD}_{3} \mathrm{OD}\right) \delta 6.76(\mathrm{~s}, 1 \mathrm{H}), 5.68(\mathrm{~d}, J=1.1 \mathrm{~Hz}, 1 \mathrm{H}), 4.25(\mathrm{dd}, J=11.6,6.7 \mathrm{~Hz}, 1 \mathrm{H})$, $3.86(\mathrm{~s}, 3 \mathrm{H}), 3.82(\mathrm{~s}, 3 \mathrm{H}), 3.73(\mathrm{~s}, 3 \mathrm{H}), 3.49(\mathrm{~s}, 3 \mathrm{H}), 3.26-3.20(\mathrm{~m}, 4 \mathrm{H}), 2.79$ (dd, J= 13.9, 8.2 Hz, $1 \mathrm{H}), 2.10-2.00(\mathrm{~m}, 1 \mathrm{H}), 1.94(\mathrm{~s}, 3 \mathrm{H}), 1.86(\mathrm{td}, J=12.2,8.6 \mathrm{~Hz}, 1 \mathrm{H}), 1.36(\mathrm{dd}, J=9.0,4.0 \mathrm{~Hz}, 1 \mathrm{H})$, $1.14(\mathrm{dd}, J=7.3,4.0 \mathrm{~Hz}, 1 \mathrm{H})$.

${ }^{13}$ C NMR (150 MHz, CD 3 OD) $\delta$ 190.9, 172.2, 171.4, 154.6, 154.2, 142.6, 138.6, 124.2, 115.9, 110.0, 97.2, 63.1, 61.6, 56.6, 52.4, 51.0, 49.3, 34.8, 33.2, 30.2, 29.8, 27.1, 22.4. 
IR (KBr): 3344, 2940, 2836, 1671, 1537, 1488, 1463, 1324, 1240, 1111, 1043, 761, $595 \mathrm{~cm}^{-1}$.

HRMS (ESI): calc. for $\mathrm{C}_{23} \mathrm{H}_{29} \mathrm{NO}_{7} \mathrm{Na}[\mathrm{M}+\mathrm{Na}]^{+}: 454.1836$, found: 454.1834 .

Optical rotation: $[\alpha]_{\mathrm{D}}^{22.9}=-63.03^{\circ}\left(c 0.54, \mathrm{CHCl}_{3}\right)$.

Data for colchicine 1

$\mathbf{R}_{\boldsymbol{f}}: 0.38$ (acetone).

Physical state: dark yellow solid.

m.p.: $144-146{ }^{\circ} \mathrm{C}$.

${ }^{1} \mathbf{H}$ NMR $\left(400 \mathrm{MHz}, \mathrm{CDCl}_{3}\right) \delta 8.53(\mathrm{~d}, J=5.9 \mathrm{~Hz}, 1 \mathrm{H}), 7.64(\mathrm{~s}, 1 \mathrm{H}), 7.33(\mathrm{~d}, J=10.7 \mathrm{~Hz}, 1 \mathrm{H}), 6.88$ $(\mathrm{d}, J=10.9 \mathrm{~Hz}, 1 \mathrm{H}), 6.51(\mathrm{~s}, 1 \mathrm{H}), 4.63 \mathrm{dt}, J=11.7,5.6 \mathrm{~Hz}, 1 \mathrm{H}), 3.99(\mathrm{~s}, 3 \mathrm{H}), 3.91(\mathrm{~s}, 3 \mathrm{H}), 3.88(\mathrm{~s}$, $3 \mathrm{H}), 3.63(\mathrm{~s}, 3 \mathrm{H}), 2.57-2.42(\mathrm{~m}, 1 \mathrm{H}), 2.41-2.26(\mathrm{~m}, 2 \mathrm{H}), 2.02-1.90(\mathrm{~m}, 1 \mathrm{H}), 1.92(\mathrm{~s}, 3 \mathrm{H})$.

${ }^{13}$ C NMR $\left(100 \mathrm{MHz} \mathrm{CDCl}_{3}\right) \delta 179.6,170.3,164.1,153.6,153.0,151.2,141.7,137.2,135.8,134.4$, $130.4,125.6,113.1,107.4,61.6,61.4,56.5,56.2,52.9,36.3,29.9,22.7$.

IR (KBr): 3431, 3281, 2938, 2839, 1664, 1616, 1589, 1564, 1488, 1252, 1140, 1094, 1019, 843, 485 $\mathrm{cm}^{-1}$.

HRMS (ESI): calc. for $\mathrm{C}_{22} \mathrm{H}_{25} \mathrm{NO}_{6} \mathrm{Na}[\mathrm{M}+\mathrm{Na}]^{+}: 422.1574$, found: 422.1576 .

Optical rotation: $[\alpha]_{\mathrm{D}}^{24.8}=-129.75^{\circ}\left(c 0.54, \mathrm{CHCl}_{3}\right)$.

HPLC analysis indicated that the enantiomeric excess of the product was $>99 \%$ [Diacel Chiralpak $\mathrm{AD}-\mathrm{H}$; hexanes $/ i$-PrOH $=80 / 20$; flow rate $=1.0 \mathrm{~mL} / \mathrm{min} ; 25^{\circ} \mathrm{C}$; detection wavelength $=210 \mathrm{~nm}$; $\mathrm{t}_{1}=$ $8.226 \mathrm{~min} ; \mathrm{t}_{2}=13.395 \min$ (major)]. 
<smiles>CCNCCN[C@H]1CCc2cc(OC)c(OC)c(OC)c2-c2ccc(OC)c(=O)cc21</smiles>

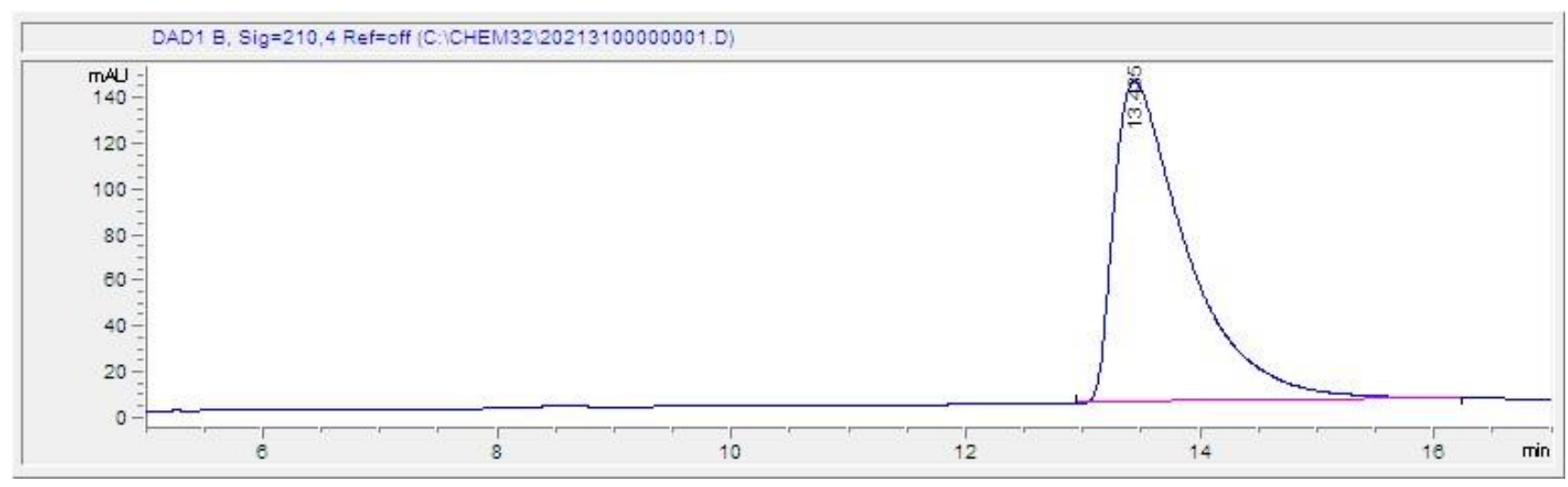

\begin{tabular}{|c|c|c|c|c|c|c|}
\hline \# & Time & Area & Height & Width & Area\% & Symmetry \\
\hline 1 & 13.435 & 6145.6 & 140.4 & 0.6386 & 100.000 & 0.352 \\
\hline
\end{tabular}

$r a c-1$

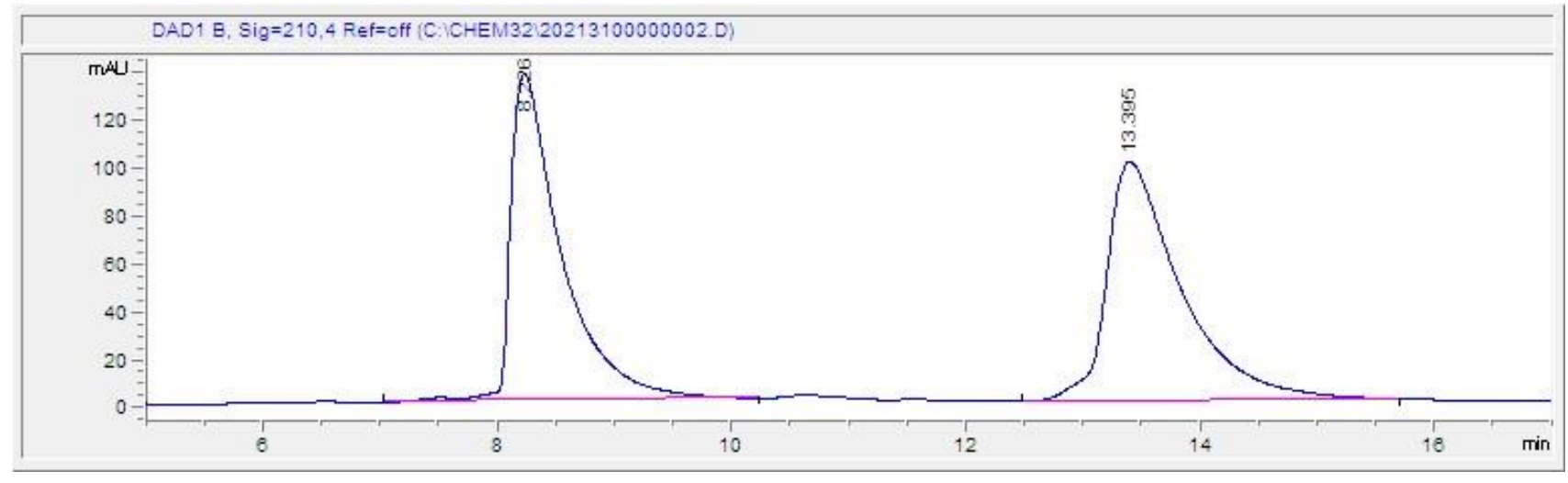

\begin{tabular}{|c|c|c|c|c|c|c|}
\multicolumn{1}{l}{ \# } & \multicolumn{1}{c}{ Time } & Area & Height & Width & \multicolumn{2}{c|}{ Area\% } \\
\hline 1 & 8.226 & 4147.4 & 136.5 & 0.4404 & 48.499 & 0.351 \\
\hline 2 & 13.395 & 4404.1 & 99.8 & 0.639 & 51.501 & 0.432 \\
\hline
\end{tabular}

Comparison of ${ }^{1}$ H NMR Data for Commercial vs. Synthetic (-)-Colchicine ${ }^{5}$ 


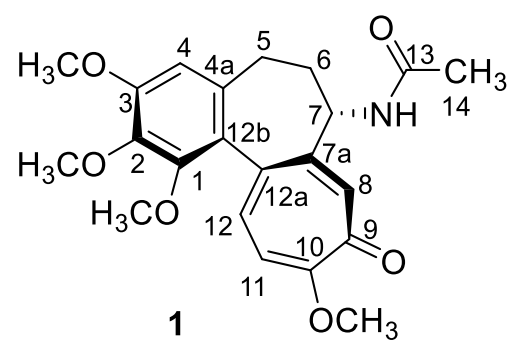

\begin{tabular}{|c|c|c|}
\hline Position & $\begin{array}{c}\text { Commercially Available (-)-Colchicine (1) } \\
\text { (Purchased from Aladdin Industrial Corporation) } \\
\qquad{ }^{1} \mathrm{H} \mathrm{NMR}, 400 \mathrm{MHz}, \mathrm{CD}_{3} \mathrm{Cl}\end{array}$ & $\begin{array}{l}\text { Synthetic (-)-Colchicine (1) } \\
{ }^{1} \mathrm{H} \text { NMR, } 400 \mathrm{MHz}, \mathrm{CD}_{3} \mathrm{Cl}\end{array}$ \\
\hline 4 & $6.52(\mathrm{~s}, 1 \mathrm{H})$ & $6.51(\mathrm{~s}, 1 \mathrm{H})$ \\
\hline 5 & $2.50(\mathrm{~m}, 1 \mathrm{H}) ; 2.35(\mathrm{~m}, 1 \mathrm{H})$ & $2.49(\mathrm{~m}, 1 \mathrm{H}) ; 2.34(\mathrm{~m}, 1 \mathrm{H})$ \\
\hline 6 & $2.35(\mathrm{~m}, 1 \mathrm{H}) ; 1.96(\mathrm{~m}, 1 \mathrm{H})$ & $2.34(\mathrm{~m}, 1 \mathrm{H}) ; 1.95(\mathrm{~m}, 1 \mathrm{H})$ \\
\hline 7 & $4.64(\mathrm{dt}, J=11.8,5.7 \mathrm{~Hz}, 1 \mathrm{H}$ & $4.63(\mathrm{dt}, J=11.7,5.6 \mathrm{~Hz}, 1 \mathrm{H})$ \\
\hline 8 & $7.63(\mathrm{~s}, 1 \mathrm{H})$ & $7.64(\mathrm{~s}, 1 \mathrm{H})$ \\
\hline 11 & $6.89(\mathrm{~d}, J=10.9 \mathrm{~Hz}, 1 \mathrm{H})$ & $6.88(\mathrm{~d}, J=10.9 \mathrm{~Hz}, 1 \mathrm{H})$ \\
\hline 12 & $7.34(\mathrm{~d}, J=10.7 \mathrm{~Hz}, 1 \mathrm{H})$ & $7.33(\mathrm{~d}, J=10.7 \mathrm{~Hz}, 1 \mathrm{H})$ \\
\hline 14 & $1.94(\mathrm{~s}, 3 \mathrm{H})$ & $1.92(\mathrm{~s}, 3 \mathrm{H})$ \\
\hline $1-\mathrm{OCH}_{3}$ & $3.64(\mathrm{~s}, 3 \mathrm{H})$ & $3.63(\mathrm{~s}, 3 \mathrm{H})$ \\
\hline $2-\mathrm{OCH}_{3}$ & $3.93(\mathrm{~s}, 3 \mathrm{H})$ & $3.91(\mathrm{~s}, 3 \mathrm{H})$ \\
\hline $3-\mathrm{OCH}_{3}$ & $3.89(\mathrm{~s}, 3 \mathrm{H})$ & $3.88(\mathrm{~s}, 3 \mathrm{H})$ \\
\hline $10-\mathrm{OCH}_{3}$ & $4.00(\mathrm{~s}, 3 \mathrm{H})$ & $3.99(\mathrm{~s}, 3 \mathrm{H})$ \\
\hline NH & $8.38(\mathrm{~d}, J=6.0 \mathrm{~Hz}, 1 \mathrm{H})$ & $8.53(\mathrm{~d}, J=5.9 \mathrm{~Hz}, 1 \mathrm{H})$ \\
\hline
\end{tabular}




\section{Comparison of ${ }^{13} \mathrm{C}$ NMR Data for Commercial vs. Synthetic (-)-Colchicine ${ }^{5}$}

\begin{tabular}{|c|c|c|}
\hline Position & $\begin{array}{c}\text { Commercially Available (-)-Colchicine (1) } \\
\text { (Purchased from Aladdin Industrial Corporation) } \\
\qquad{ }^{1} \mathrm{H} \mathrm{NMR}, 400 \mathrm{MHz}, \mathrm{CD}_{3} \mathrm{Cl}\end{array}$ & $\begin{array}{l}\text { Synthetic (-)-Colchicine (1) } \\
{ }^{1} \mathrm{H} \mathrm{NMR}, 400 \mathrm{MHz}, \mathrm{CD}_{3} \mathrm{Cl}\end{array}$ \\
\hline 1 & 151.3 & 151.2 \\
\hline 2 & 141.7 & 141.7 \\
\hline 3 & 153.6 & 153.6 \\
\hline 4 & 107.4 & 107.3 \\
\hline $4 a$ & 134.4 & 134.4 \\
\hline 5 & 30.0 & 29.9 \\
\hline 6 & 36.4 & 36.3 \\
\hline 7 & 52.9 & 52.9 \\
\hline $7 \mathrm{a}$ & 152.9 & 153.0 \\
\hline 8 & 130.5 & 130.4 \\
\hline 9 & 179.6 & 179.6 \\
\hline 10 & 164.1 & 164.1 \\
\hline 11 & 113.1 & 113.1 \\
\hline 12 & 135.8 & 135.8 \\
\hline $12 \mathrm{a}$ & 137.2 & 137.2 \\
\hline $12 b$ & 125.7 & 125.6 \\
\hline 13 & 170.3 & 170.3 \\
\hline 14 & 22.8 & 22.7 \\
\hline $1-\mathrm{OCH}_{3}$ & 61.7 & 61.6 \\
\hline $2-\mathrm{OCH}_{3}$ & 61.5 & 61.4 \\
\hline $3-\mathrm{OCH}_{3}$ & 56.2 & 56.2 \\
\hline $10-\mathrm{OCH}_{3}$ & 56.6 & 56.5 \\
\hline
\end{tabular}




\section{References}

[1] Difieber, C.; Ariger, M. A.; Moriel, P.; Carreira, E. M. Angew. Chem. Int. Ed., 2007, 46, 3139.

[2] Lafrance, M.; Roggen, M.; Carreira, E. M. Angew. Chem. Int. Ed., 2012, 51, 3470.

[3] Geoghegan, K.; Kelleher, S.; Evans, P. J. Org. Chem., 2011, 76, 2187.

[4] Takubo, K.; Furutsu, K.; Ide, T.; Nemoto, H., Ueda, Y.; Tsujikawa, K.; Ikawa, T.; Yoshimitsu, T.; Akai, S. Eur. J. Org. Chem., 2016, 8, 1562.

[5] Meksuriyen, D.; Lin, L. J.; Cordell, G. A.; Mukhopadhyay, S.; Banerjee, S. K. J. Nat. Prod., 1988, 51,88 . 


\section{NMR Spectra}

学

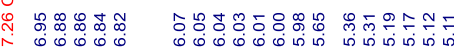

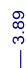

\section{$\stackrel{\text { ๘ }}{\text { i }}$}

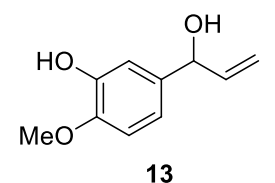

${ }^{1} \mathrm{H}$ NMR $400 \mathrm{MHz} \mathrm{CDCl}_{3}$
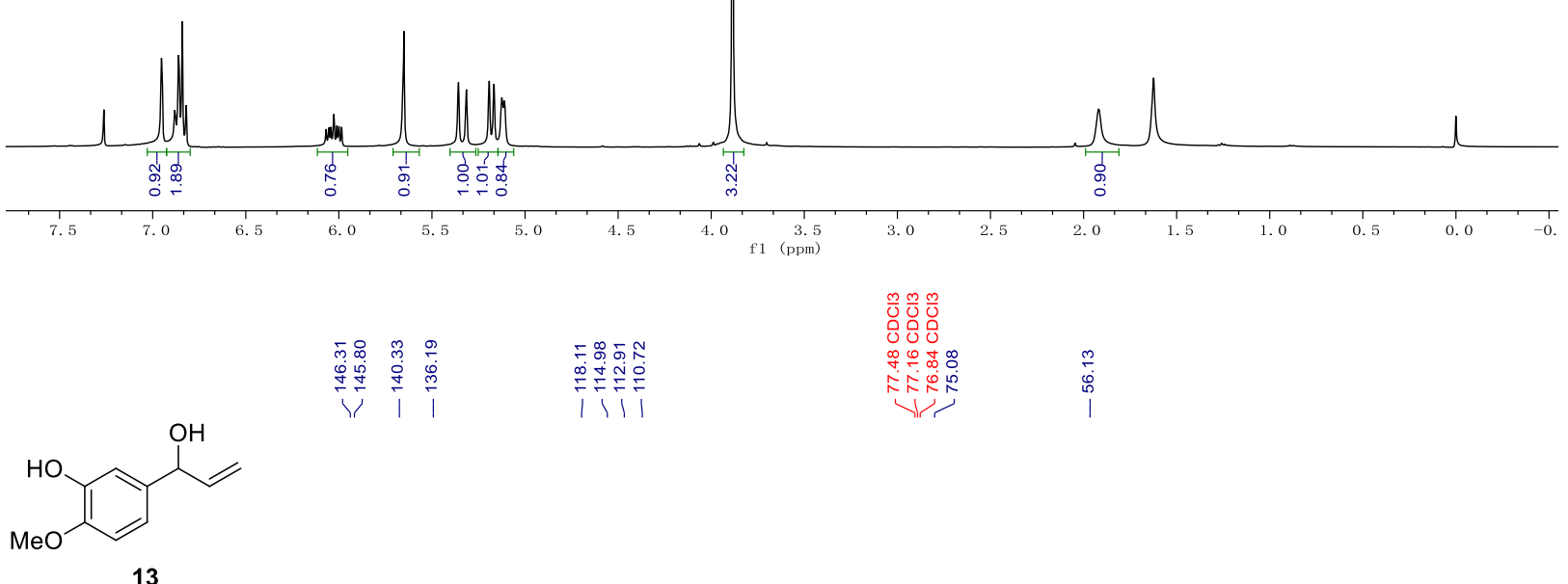

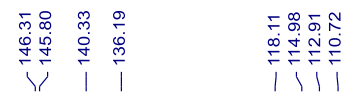

$\frac{m}{0} \frac{m}{0}$
000
000

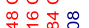

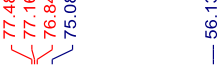

\section{i.}

${ }^{13} \mathrm{C}$ NMR $100 \mathrm{MHz} \mathrm{CDCl}_{3}$

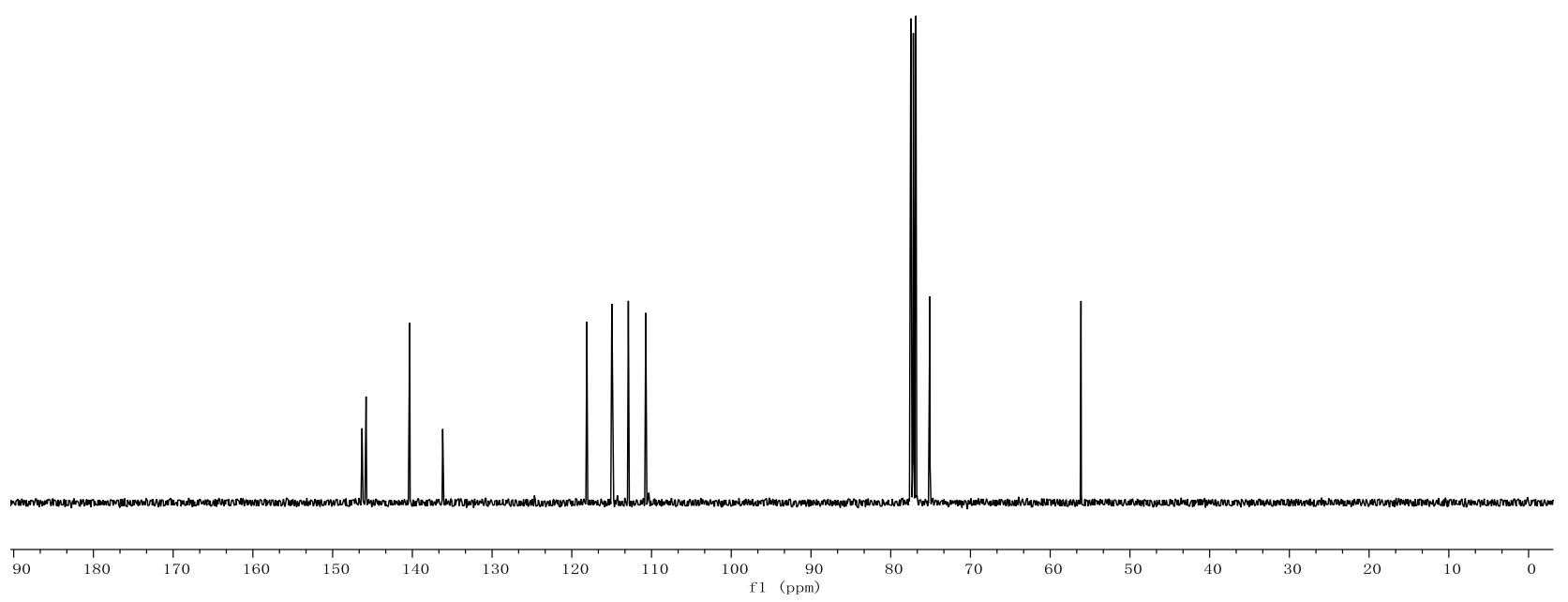


离

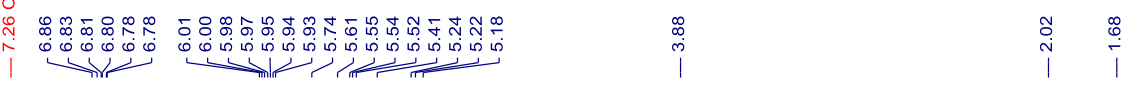

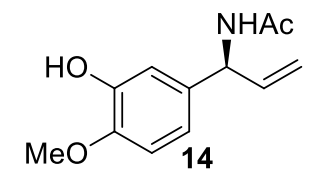

${ }^{1} \mathrm{H}$ NMR $400 \mathrm{MHz} \mathrm{CDCl}_{3}$
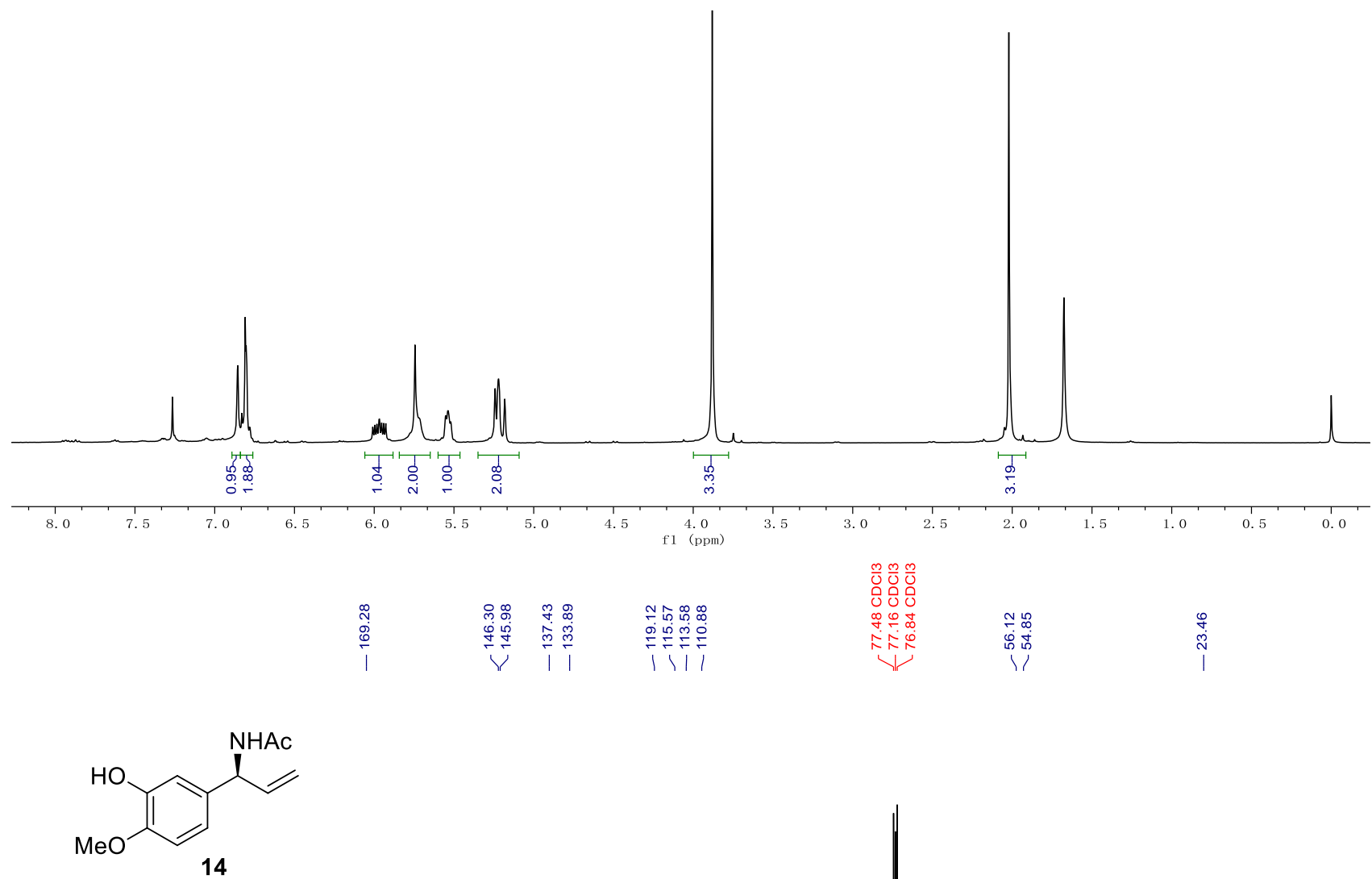

${ }^{13} \mathrm{C}$ NMR $100 \mathrm{MHz} \mathrm{CDCl}_{3}$

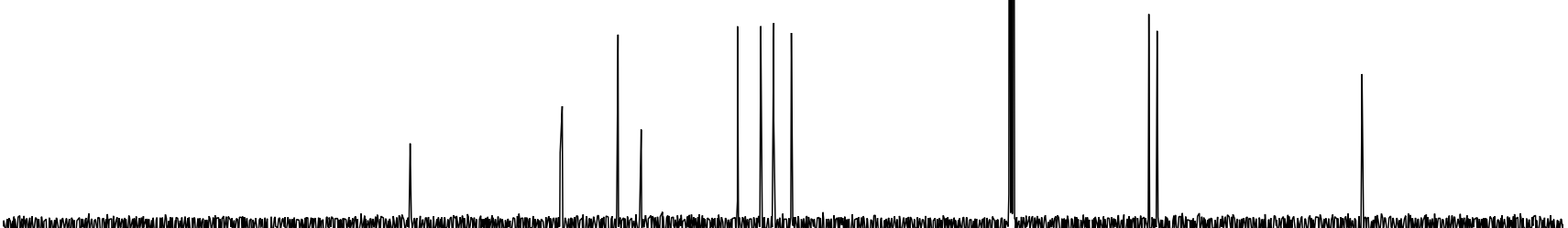

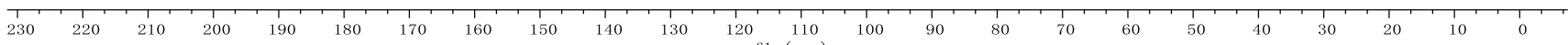


$\frac{m}{0}$

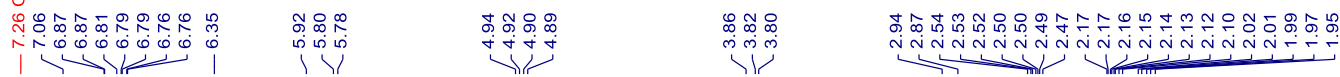<smiles>COc1ccc([C@H](N)CCc2cc(OC)c(OC)c(OC)c2)cc1O</smiles>

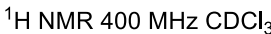

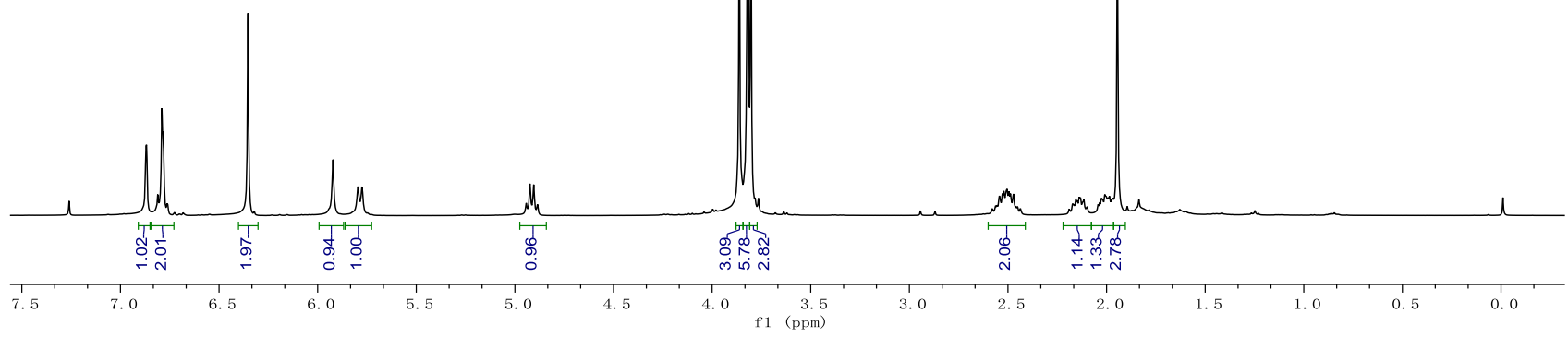

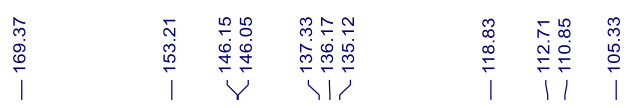

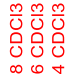

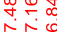

走采

무요

००

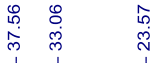

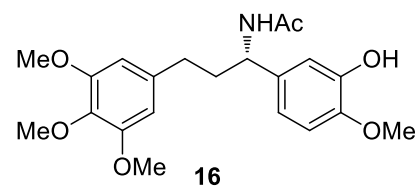

${ }^{13} \mathrm{C} \mathrm{NMR} 100 \mathrm{MHz} \mathrm{CDCl}_{3}$

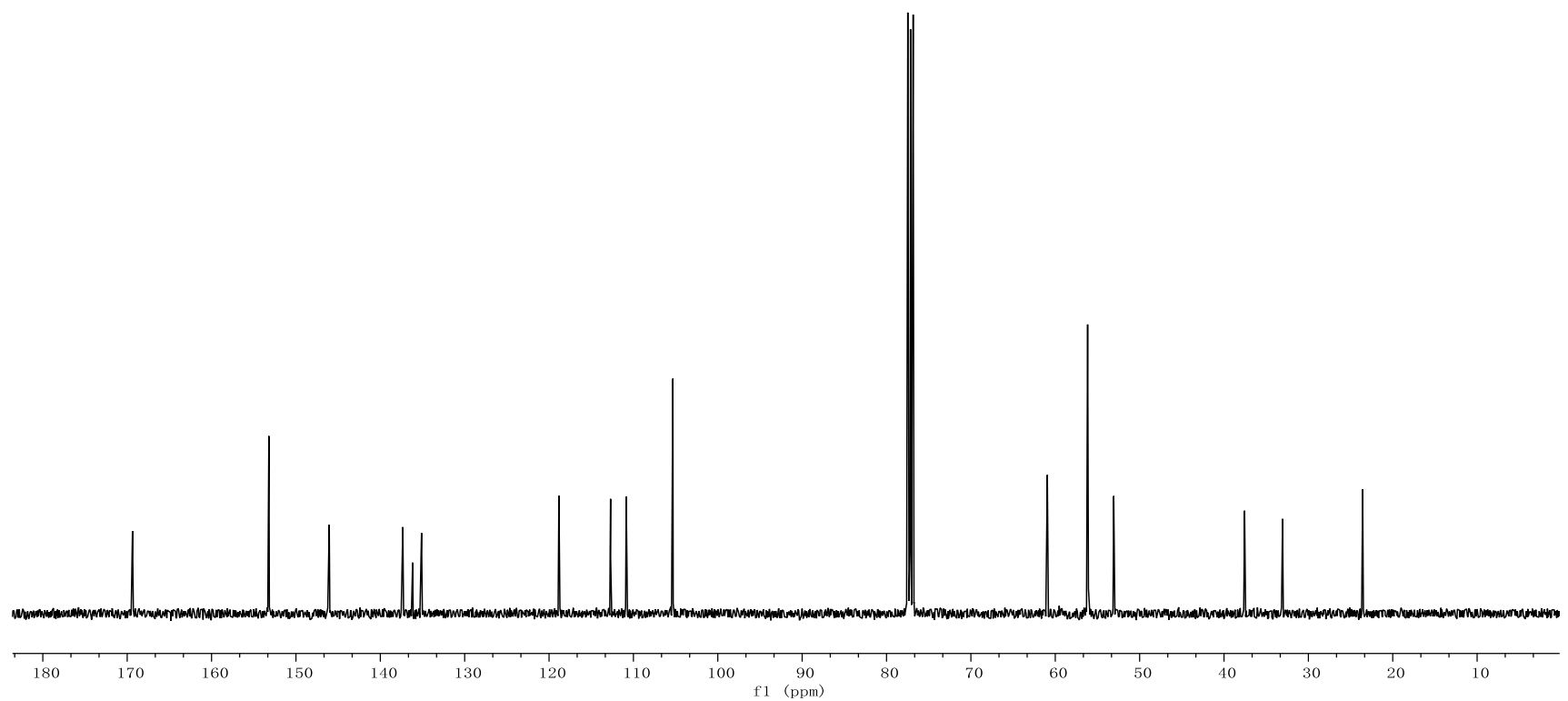




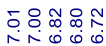

Yरण

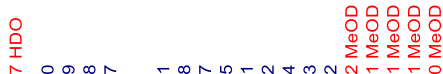

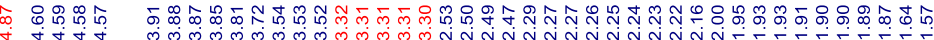

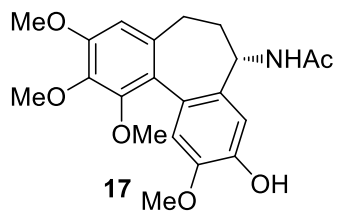

${ }^{1} \mathrm{H} \mathrm{NMR} 600 \mathrm{MHz} \mathrm{CD}_{3} \mathrm{OD}$
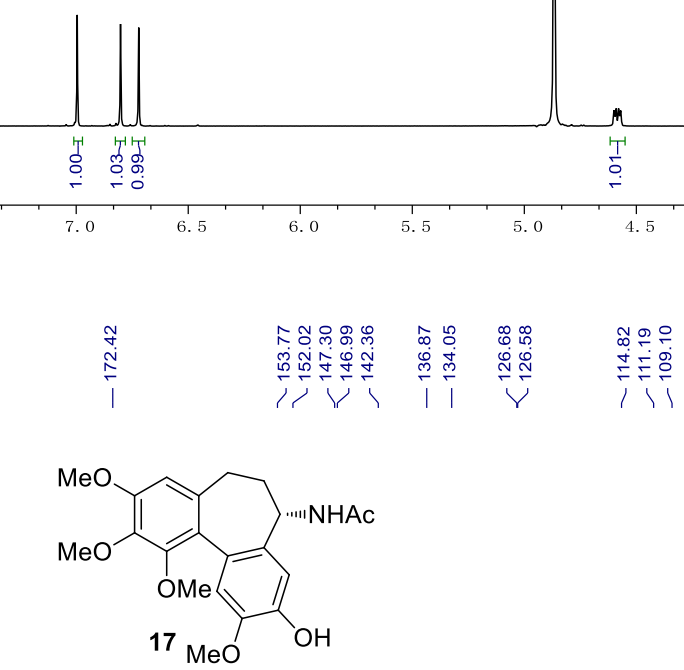

${ }^{13} \mathrm{C}$ NMR $150 \mathrm{MHz} \mathrm{CD}_{3} \mathrm{OD}$

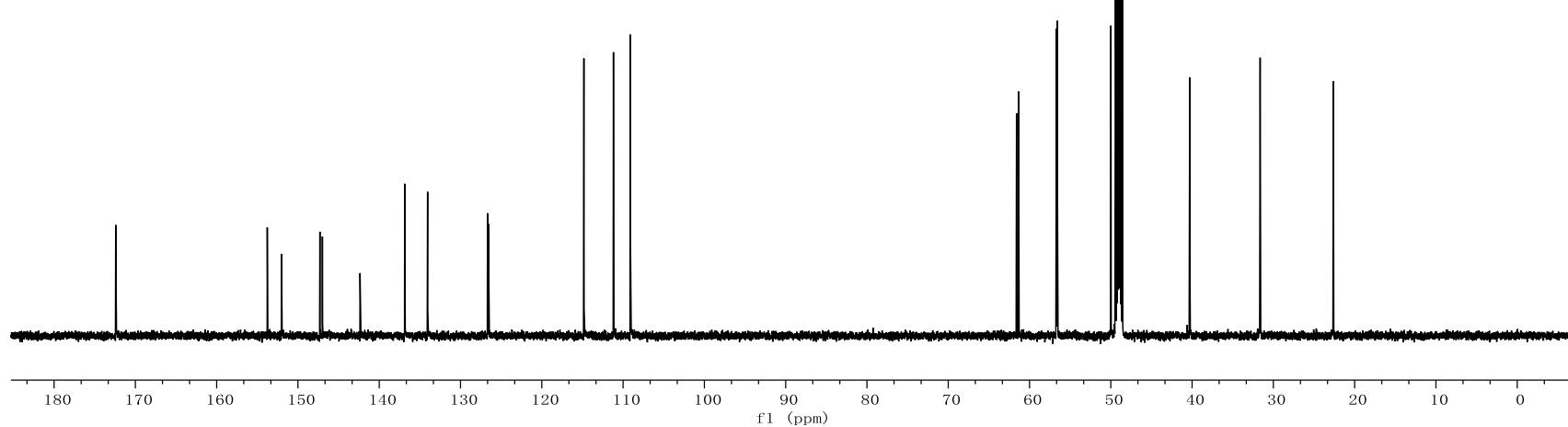




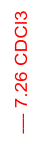

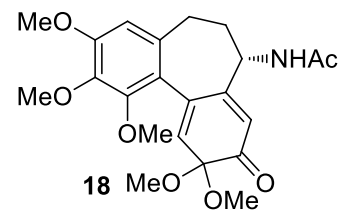

${ }^{1} \mathrm{H}$ NMR $400 \mathrm{MHz} \mathrm{CDCl}_{3}$

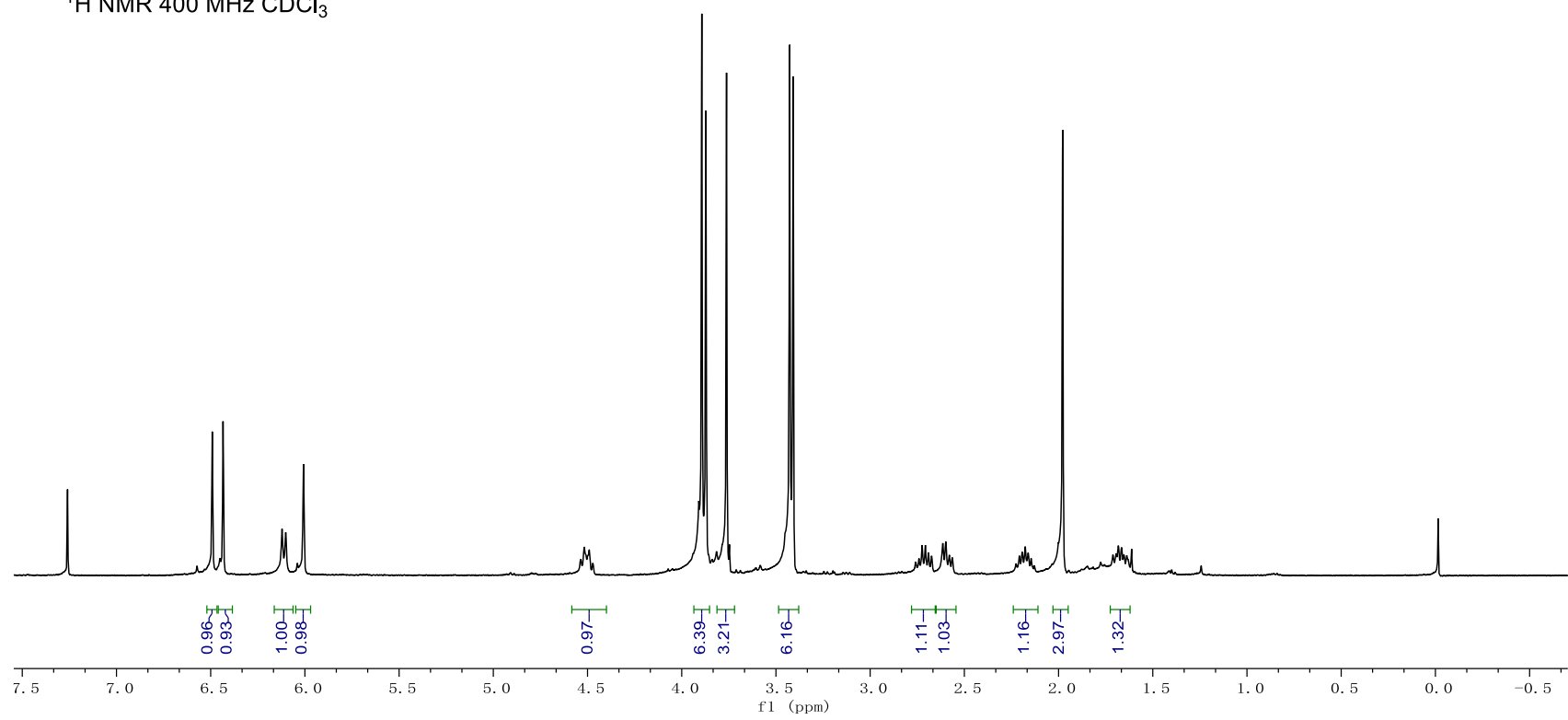

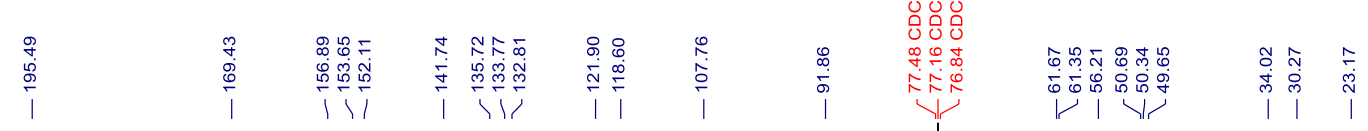

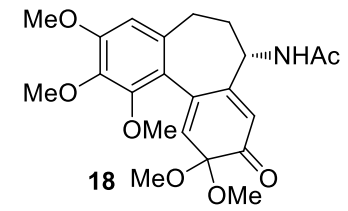

${ }^{13} \mathrm{C}$ NMR $100 \mathrm{MHz} \mathrm{CDCl}_{3}$

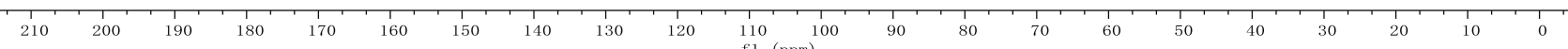




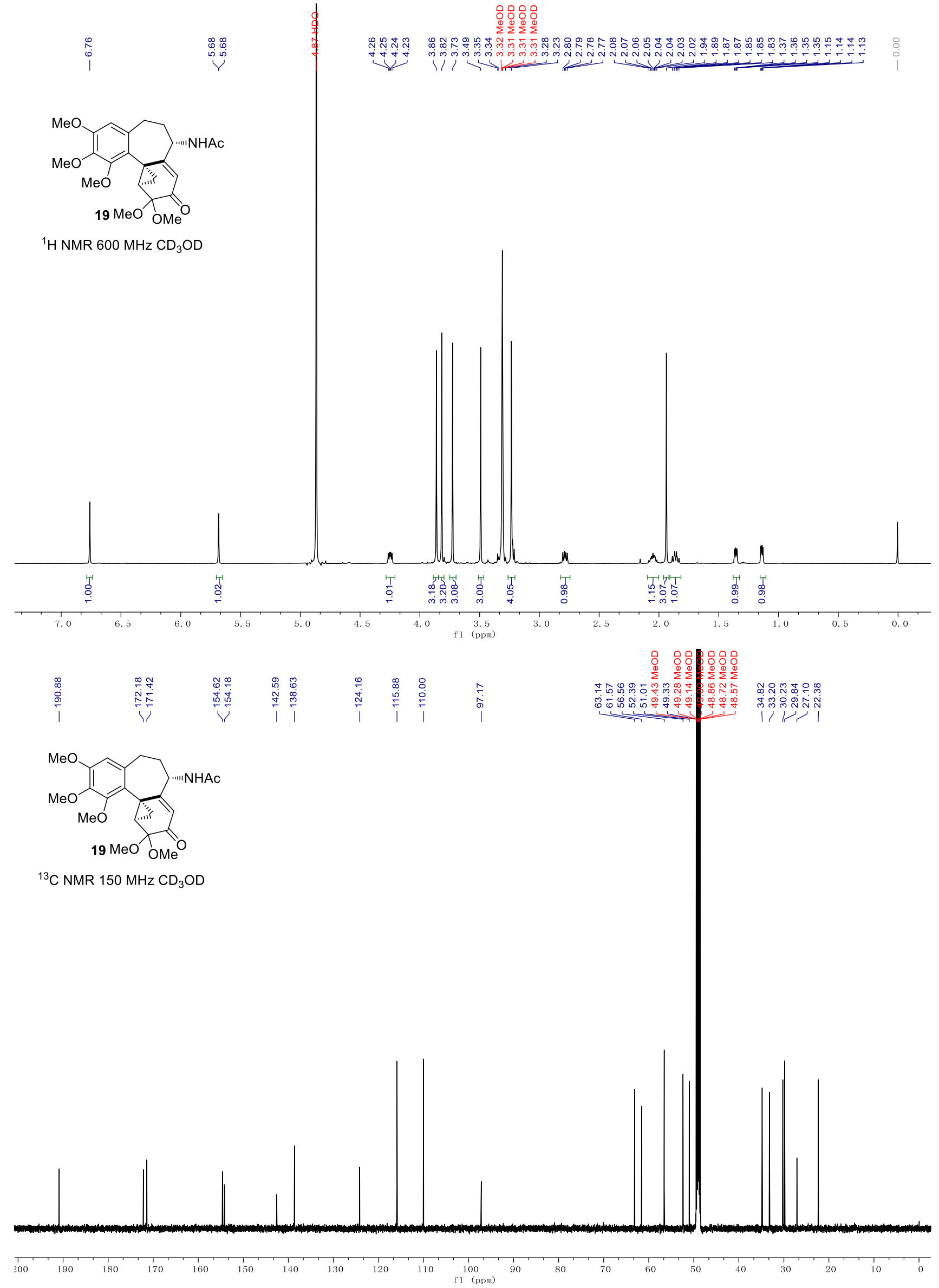



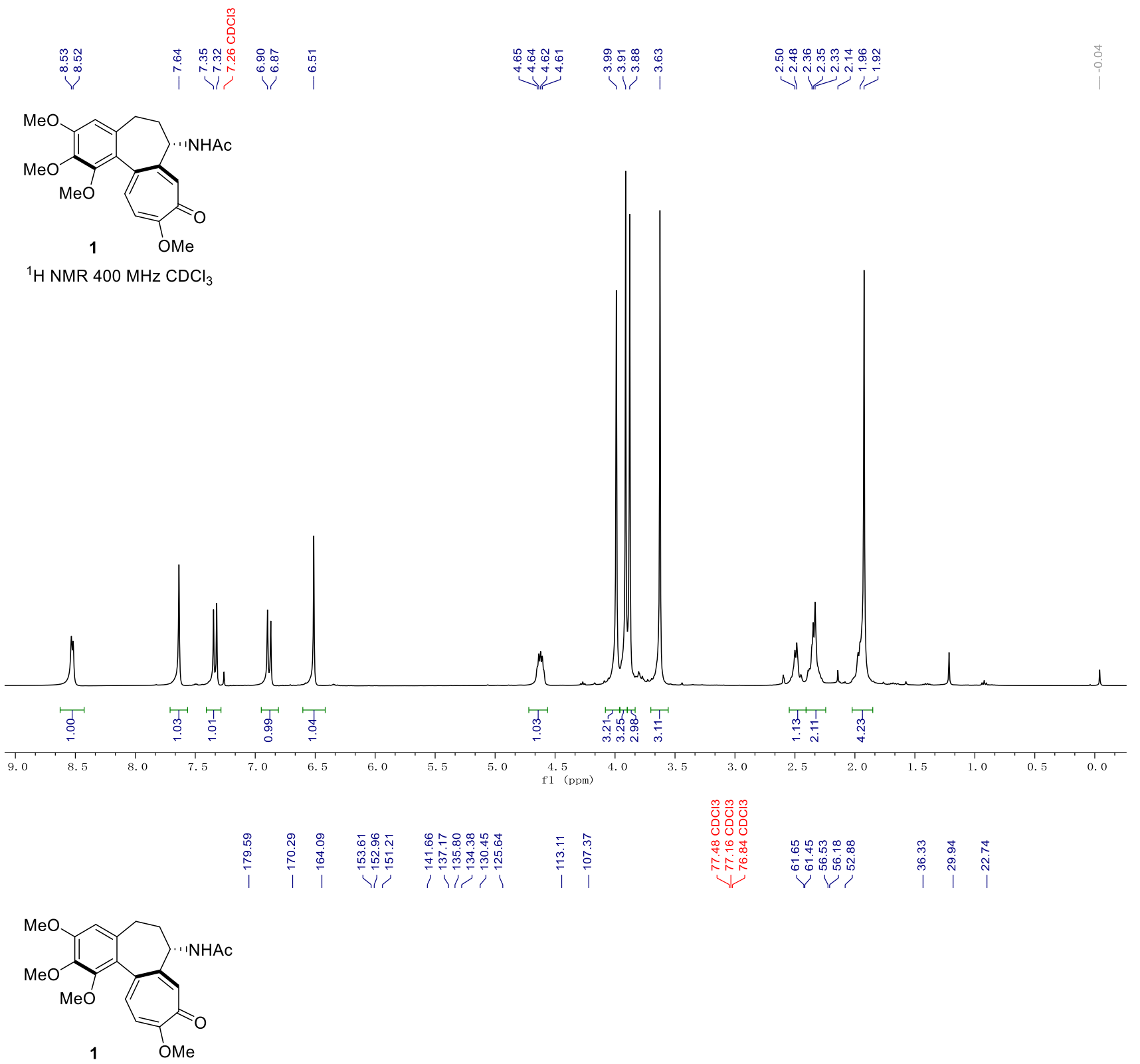

${ }^{13} \mathrm{C}$ NMR $100 \mathrm{MHz} \mathrm{CDCl}_{3}$

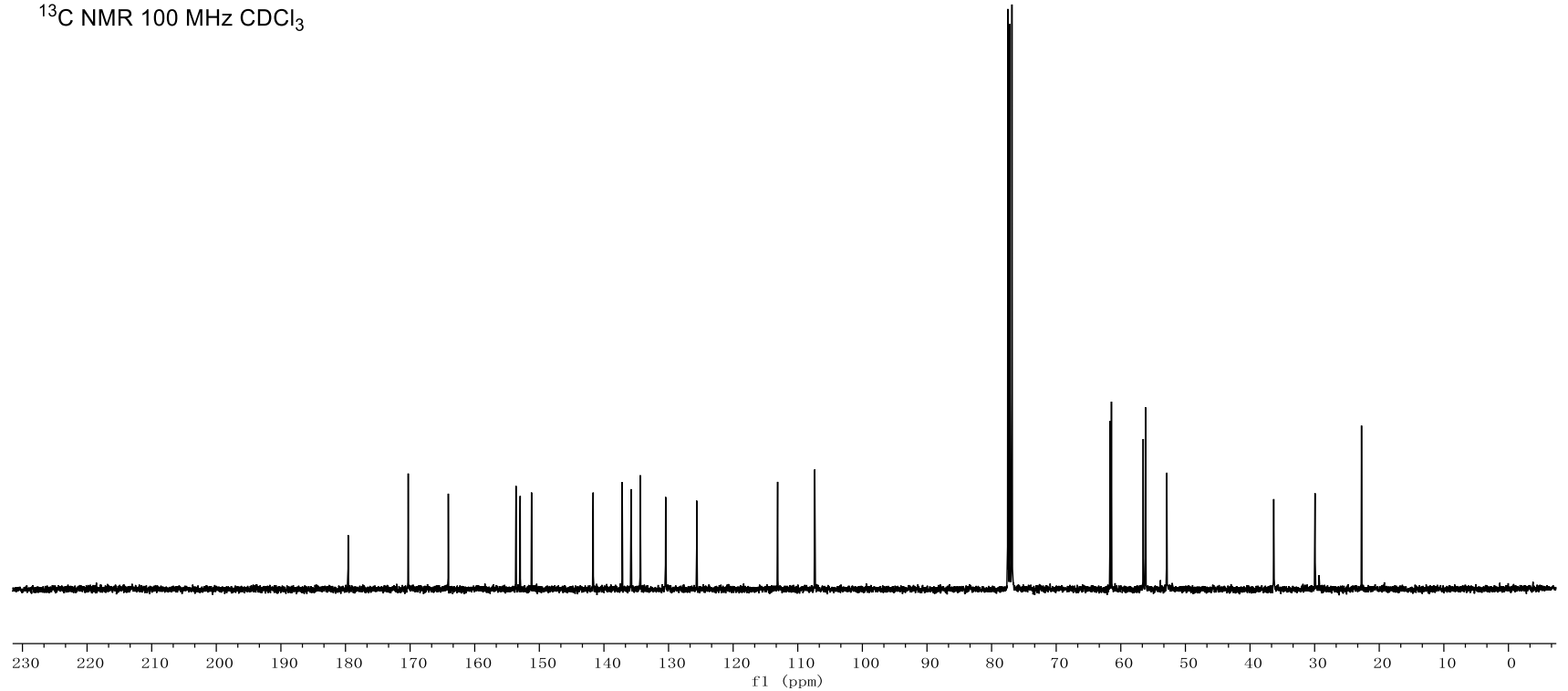



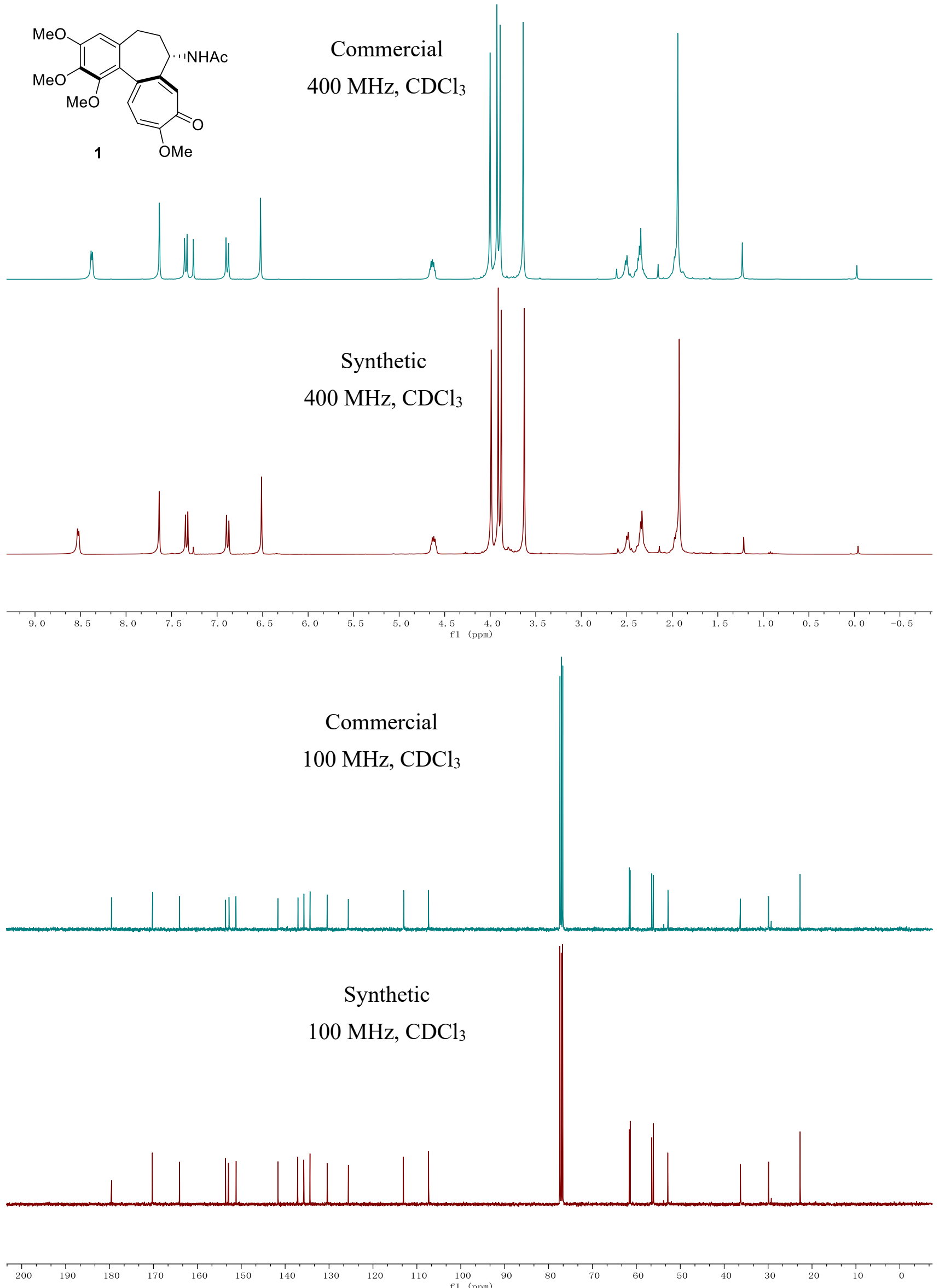
Crystal data for compound 17: $\mathrm{C}_{21} \mathrm{H}_{25} \mathrm{NO}_{6} \cdot \mathrm{C}_{3} \mathrm{H}_{6} \mathrm{O}, M=445.50, a=10.0751(2) \AA ⿻, b=11.1470(2)$ $\AA, c=10.2012(2) \AA, \alpha=90^{\circ}, \beta=93.3300(10)^{\circ}, \gamma=90^{\circ}, V=1143.73(4) \AA^{3}, T=100(2) \mathrm{K}$, space group $P 21, Z=2, \mu(\mathrm{CuK} \alpha)=0.783 \mathrm{~mm}^{-1}, 11302$ reflections measured, 3768 independent reflections $\left(R_{\text {int }}=\right.$ $0.0353)$. The final $R_{l}$ values were $0.0346(I>2 \sigma(I))$. The final $w R\left(F^{2}\right)$ values were $0.0906(I>2 \sigma(I))$. The final $R_{1}$ values were 0.0346 (all data). The final $w R\left(F^{2}\right)$ values were 0.0906 (all data). The goodness of fit on $F^{2}$ was 1.054. Flack parameter $=0.11(4)$.

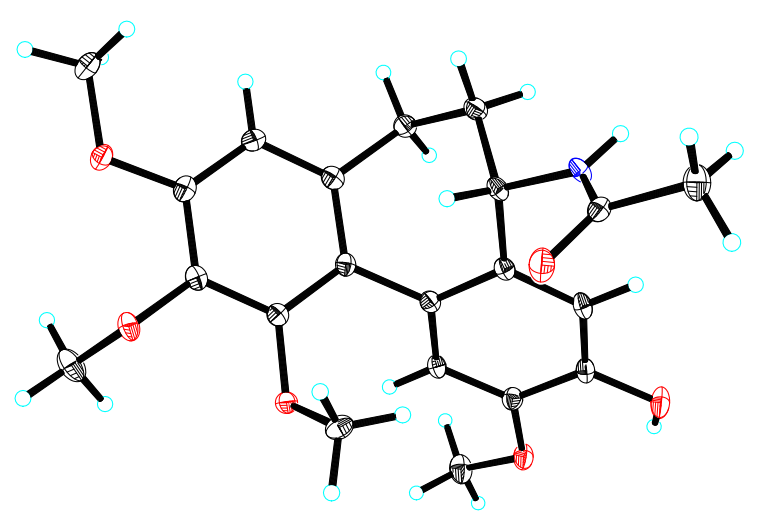

Displacement ellipsoids are drawn at the $30 \%$ probability level.

Table 1 Crystal data and structure refinement for compound 17.

Identification code

Empirical formula

Formula weight

Temperature/K

Wavelength

Crystal system

Space group

Unit cell dimensions

Volume

Z cu_af16070_0m

$\mathrm{C}_{24} \mathrm{H}_{31} \mathrm{NO}_{7}$

445.50

100

$1.54178 \AA$

Monoclinic

P21

$$
\begin{array}{ll}
\mathrm{a}=10.0751(2) \AA & \alpha=90^{\circ} . \\
\mathrm{b}=11.1470(2) \AA & \beta=93.3300(10)^{\circ} . \\
\mathrm{c}=10.2012(2) \AA & \gamma=90^{\circ} .
\end{array}
$$


Density (calculated)

Absorption coefficient

$\mathrm{F}(000)$

Crystal size

Theta range for data collection

Index ranges

Reflections collected

Independent reflections

Completeness to theta $=67.679^{\circ}$

Absorption correction

Refinement method

Data/restraints/parameters

Goodness-of-fit on $\mathrm{F}^{2}$

Final $\mathrm{R}$ indices [I $>2 \operatorname{sigma}(\mathrm{I})]$

$\mathrm{R}$ indices (all data)

Absolute structure parameter

Extinction coefficient

Largest diff. peak and hole
$1.294 \mathrm{~g} / \mathrm{cm}^{3}$

$0.783 \mathrm{~mm}^{-1}$

476

$0.850 \times 0.640 \times 0.340 \mathrm{~mm}^{3}$

4.341 to $69.270^{\circ}$.

$-12<=\mathrm{h}<=11,-13<=\mathrm{k}<=12,-10<=\mathrm{l}<=11$

11302

$3768\left[\mathrm{R}_{\text {int }}=0.0353\right]$

$96.0 \%$

Semi-empirical from equivalents

Full-matrix least-squares on $\mathrm{F}^{2}$

$3768 / 1 / 298$

1.054

$\mathrm{R}_{1}=0.0346, \mathrm{w}_{2}=0.0906$

$\mathrm{R}_{1}=0.0346, \mathrm{wR}_{2}=0.0906$

$0.11(4)$

$0.0206(15)$

0.254 and -0.224 e $\AA^{-3}$ 
Table 2 Atomic Coordinates $\left(\times 1^{4}\right)$ and Equivalent Isotropic Displacement Parameters $\left(\AA^{2} \times\right.$ $10^{3}$ ) for cu_af16070_0m. Ueq is defined as one third of the trace of the orthogonalized UIJ tensor.

\begin{tabular}{|c|c|c|c|c|}
\hline & $\mathrm{x}$ & $\mathbf{y}$ & $\mathbf{z}$ & $\mathrm{U}(\mathrm{eq})$ \\
\hline $\mathrm{N}(1)$ & 1767(2) & $-294(2)$ & $3173(2)$ & $22(1)$ \\
\hline $\mathrm{O}(2)$ & $2412(2)$ & $1630(2)$ & $3163(2)$ & $33(1)$ \\
\hline $\mathrm{O}(3)$ & $-2211(2)$ & $2130(2)$ & $8235(2)$ & $26(1)$ \\
\hline $\mathrm{O}(4)$ & 2447(1) & $2030(2)$ & $8206(2)$ & $22(1)$ \\
\hline $\mathrm{O}(5)$ & 5913(2) & $-1824(2)$ & $5590(2)$ & $32(1)$ \\
\hline $\mathrm{O}(6)$ & $5721(2)$ & $-1398(2)$ & $8166(2)$ & $28(1)$ \\
\hline $\mathrm{O}(7)$ & $5811(4)$ & $873(4)$ & 1692(4) & $119(2)$ \\
\hline $\mathrm{O}(1)$ & $138(2)$ & $3176(2)$ & $8692(2)$ & $24(1)$ \\
\hline $\mathrm{C}(1)$ & 194(3) & $3283(3)$ & $10087(3)$ & $36(1)$ \\
\hline $\mathrm{C}(2)$ & $100(2)$ & 2024(2) & $8214(2)$ & $20(1)$ \\
\hline $\mathrm{C}(3)$ & $1267(2)$ & $1450(2)$ & $7888(2)$ & $18(1)$ \\
\hline $\mathrm{C}(4)$ & $1224(2)$ & $343(2)$ & $7240(2)$ & 19(1) \\
\hline$C(5)$ & 2461(2) & $-253(2)$ & $6830(2)$ & $20(1)$ \\
\hline$C(6)$ & 2584(2) & $-526(2)$ & $5500(2)$ & 19(1) \\
\hline $\mathrm{C}(7)$ & $1414(2)$ & $-272(2)$ & $4535(2)$ & $20(1)$ \\
\hline $\mathrm{C}(8)$ & $2249(2)$ & $672(2)$ & 2581(2) & $21(1)$ \\
\hline $\mathrm{C}(9)$ & $-3492(2)$ & 1617(3) & 7908(3) & $35(1)$ \\
\hline$C(10)$ & $-1130(2)$ & $1474(2)$ & 7904(2) & $22(1)$ \\
\hline$C(11)$ & $3038(2)$ & $2540(2)$ & $7080(2)$ & $25(1)$ \\
\hline$C(12)$ & $-14(2)$ & $-180(2)$ & 6913(2) & $20(1)$ \\
\hline$C(13)$ & $-66(2)$ & $-1343(2)$ & $6145(2)$ & $23(1)$ \\
\hline$C(14)$ & 266(2) & $-1156(2)$ & $4707(2)$ & $24(1)$ \\
\hline$C(15)$ & 2570(3) & $505(2)$ & 1175(3) & $31(1)$ \\
\hline$C(16)$ & $3757(2)$ & $-1042(2)$ & $5114(2)$ & $23(1)$ \\
\hline
\end{tabular}




\begin{tabular}{lllll}
\hline $\mathrm{C}(17)$ & $4788(2)$ & $-1321(2)$ & $6021(2)$ & $22(1)$ \\
$\mathrm{C}(18)$ & $4653(2)$ & $-1078(2)$ & $7350(2)$ & $22(1)$ \\
$\mathrm{C}(19)$ & $5509(3)$ & $-1472(3)$ & $9524(3)$ & $34(1)$ \\
$\mathrm{C}(20)$ & $3503(2)$ & $-530(2)$ & $7747(2)$ & $20(1)$ \\
$\mathrm{C}(21)$ & $-1186(2)$ & $378(2)$ & $7257(2)$ & $21(1)$ \\
$\mathrm{C}(22)$ & $8115(4)$ & $778(4)$ & $1831(4)$ & $61(1)$ \\
$\mathrm{C}(23)$ & $6799(4)$ & $854(3)$ & $2398(4)$ & $53(1)$ \\
$\mathrm{C}(24)$ & $6753(4)$ & $876(3)$ & $3866(4)$ & $57(1)$ \\
\hline
\end{tabular}

Table 3 Bond Lengths $[\AA ̊ \cap]$ and Angles $\left[{ }^{\circ}\right]$ for Compound 17

\begin{tabular}{llll}
\hline $\mathrm{N}(1)-\mathrm{C}(8)$ & $1.339(3)$ & $\mathrm{N}(1)-\mathrm{C}(7)$ & $1.455(3)$ \\
$\mathrm{N}(1)-\mathrm{H}(1)$ & 0.8800 & $\mathrm{O}(2)-\mathrm{C}(8)$ & $1.228(3)$ \\
$\mathrm{O}(3)-\mathrm{C}(10)$ & $1.370(3)$ & $\mathrm{O}(3)-\mathrm{C}(9)$ & $1.433(3)$ \\
$\mathrm{O}(4)-\mathrm{C}(3)$ & $1.376(3)$ & $\mathrm{O}(4)-\mathrm{C}(11)$ & $1.441(3)$ \\
$\mathrm{O}(5)-\mathrm{C}(17)$ & $1.360(3)$ & $\mathrm{O}(5)-\mathrm{H}(5)$ & 0.8400 \\
$\mathrm{O}(6)-\mathrm{C}(18)$ & $1.369(3)$ & $\mathrm{O}(6)-\mathrm{C}(19)$ & $1.416(3)$ \\
$\mathrm{O}(7)-\mathrm{C}(23)$ & $1.193(4)$ & $\mathrm{O}(1)-\mathrm{C}(2)$ & $1.373(3)$ \\
$\mathrm{O}(1)-\mathrm{C}(1)$ & $1.426(3)$ & $\mathrm{C}(1)-\mathrm{H}(1 \mathrm{~A})$ & 0.9800 \\
$\mathrm{C}(1)-\mathrm{H}(1 \mathrm{~B})$ & $\mathrm{C}(1)-\mathrm{H}(1 \mathrm{C})$ & 0.9800 \\
$\mathrm{C}(2)-\mathrm{C}(3)$ & 0.9800 & $\mathrm{C}(2)-\mathrm{C}(10)$ & $1.402(3)$ \\
$\mathrm{C}(3)-\mathrm{C}(4)$ & $1.396(3)$ & $\mathrm{C}(4)-\mathrm{C}(12)$ & $1.399(3)$ \\
$\mathrm{C}(4)-\mathrm{C}(5)$ & $1.399(3)$ & $\mathrm{C}(5)-\mathrm{C}(20)$ & $1.400(3)$ \\
$\mathrm{C}(5)-\mathrm{C}(6)$ & $1.493(3)$ & $\mathrm{C}(6)-\mathrm{C}(16)$ & $1.391(3)$ \\
$\mathrm{C}(6)-\mathrm{C}(7)$ & $1.402(3)$ & $\mathrm{C}(7)-\mathrm{C}(14)$ & $1.537(3)$ \\
$\mathrm{C}(7)-\mathrm{H}(7)$ & $1.519(3)$ & $\mathrm{C}(8)-\mathrm{C}(15)$ & $1.501(3)$ \\
$\mathrm{C}(9)-\mathrm{H}(9 \mathrm{~A})$ & 1.0000 & $\mathrm{C}(9)-\mathrm{H}(9 \mathrm{~B})$ & 0.9800 \\
$\mathrm{C}(9)-\mathrm{H}(9 \mathrm{C})$ & 0.9800 & 0.9800 \\
$\mathrm{C}(11)-\mathrm{H}(11 \mathrm{~A})$ & 0.9800 & \\
\hline & & & $1.388(3)-\mathrm{H}(111 \mathrm{~B})$ \\
\hline
\end{tabular}




\begin{tabular}{|c|c|c|c|}
\hline $\mathrm{C}(11)-\mathrm{H}(11 \mathrm{C})$ & 0.9800 & $\mathrm{C}(12)-\mathrm{C}(21)$ & $1.397(3)$ \\
\hline $\mathrm{C}(12)-\mathrm{C}(13)$ & $1.514(3)$ & $\mathrm{C}(13)-\mathrm{C}(14)$ & $1.538(3)$ \\
\hline $\mathrm{C}(13)-\mathrm{H}(13 \mathrm{~A})$ & 0.9900 & $\mathrm{C}(13)-\mathrm{H}(13 \mathrm{~B})$ & 0.9900 \\
\hline $\mathrm{C}(14)-\mathrm{H}(14 \mathrm{~A})$ & 0.9900 & $\mathrm{C}(14)-\mathrm{H}(14 \mathrm{~B})$ & 0.9900 \\
\hline $\mathrm{C}(15)-\mathrm{H}(15 \mathrm{~A})$ & 0.9800 & $\mathrm{C}(15)-\mathrm{H}(15 \mathrm{~B})$ & 0.9800 \\
\hline $\mathrm{C}(15)-\mathrm{H}(15 \mathrm{C})$ & 0.9800 & $C(16)-C(17)$ & $1.385(3)$ \\
\hline $\mathrm{C}(16)-\mathrm{H}(16)$ & 0.9500 & $\mathrm{C}(17)-\mathrm{C}(18)$ & $1.398(3)$ \\
\hline $\mathrm{C}(18)-\mathrm{C}(20)$ & $1.391(3)$ & $\mathrm{C}(19)-\mathrm{H}(19 \mathrm{~A})$ & 0.9800 \\
\hline C(19)-H(19B) & 0.9800 & $\mathrm{C}(19)-\mathrm{H}(19 \mathrm{C})$ & 0.9800 \\
\hline $\mathrm{C}(20)-\mathrm{H}(20)$ & 0.9500 & $\mathrm{C}(21)-\mathrm{H}(21)$ & 0.9500 \\
\hline$C(22)-C(23)$ & $1.479(6)$ & $\mathrm{C}(22)-\mathrm{H}(22 \mathrm{~A})$ & 0.9800 \\
\hline $\mathrm{C}(22)-\mathrm{H}(22 \mathrm{~B})$ & 0.9800 & $\mathrm{C}(22)-\mathrm{H}(22 \mathrm{C})$ & 0.9800 \\
\hline$C(23)-C(24)$ & $1.501(5)$ & $\mathrm{C}(24)-\mathrm{H}(24 \mathrm{~A})$ & 0.9800 \\
\hline $\mathrm{C}(24)-\mathrm{H}(24 \mathrm{~B})$ & 0.9800 & $\mathrm{C}(24)-\mathrm{H}(24 \mathrm{C})$ & 0.9800 \\
\hline $\mathrm{C}(8)-\mathrm{N}(1)-\mathrm{C}(7)$ & $122.12(19)$ & $\mathrm{C}(8)-\mathrm{N}(1)-\mathrm{H}(1)$ & 118.9 \\
\hline $\mathrm{C}(7)-\mathrm{N}(1)-\mathrm{H}(1)$ & 118.9 & $\mathrm{C}(10)-\mathrm{O}(3)-\mathrm{C}(9)$ & $116.6(2)$ \\
\hline $\mathrm{C}(3)-\mathrm{O}(4)-\mathrm{C}(11)$ & $112.87(17)$ & $\mathrm{C}(17)-\mathrm{O}(5)-\mathrm{H}(5)$ & 109.5 \\
\hline $\mathrm{C}(18)-\mathrm{O}(6)-\mathrm{C}(19)$ & $116.85(18)$ & $\mathrm{C}(2)-\mathrm{O}(1)-\mathrm{C}(1)$ & $115.55(18)$ \\
\hline $\mathrm{O}(1)-\mathrm{C}(1)-\mathrm{H}(1 \mathrm{~A})$ & 109.5 & $\mathrm{O}(1)-\mathrm{C}(1)-\mathrm{H}(1 \mathrm{~B})$ & 109.5 \\
\hline $\mathrm{H}(1 \mathrm{~A})-\mathrm{C}(1)-\mathrm{H}(1 \mathrm{~B})$ & 109.5 & $\mathrm{O}(1)-\mathrm{C}(1)-\mathrm{H}(1 \mathrm{C})$ & 109.5 \\
\hline $\mathrm{H}(1 \mathrm{~A})-\mathrm{C}(1)-\mathrm{H}(1 \mathrm{C})$ & 109.5 & $\mathrm{H}(1 \mathrm{~B})-\mathrm{C}(1)-\mathrm{H}(1 \mathrm{C})$ & 109.5 \\
\hline $\mathrm{O}(1)-\mathrm{C}(2)-\mathrm{C}(3)$ & $120.46(19)$ & $\mathrm{O}(1)-\mathrm{C}(2)-\mathrm{C}(10)$ & $119.65(19)$ \\
\hline$C(3)-C(2)-C(10)$ & $119.4(2)$ & $\mathrm{O}(4)-\mathrm{C}(3)-\mathrm{C}(2)$ & $117.19(19)$ \\
\hline $\mathrm{O}(4)-\mathrm{C}(3)-\mathrm{C}(4)$ & 121.91(18) & $\mathrm{C}(2)-\mathrm{C}(3)-\mathrm{C}(4)$ & $120.9(2)$ \\
\hline $\mathrm{C}(12)-\mathrm{C}(4)-\mathrm{C}(3)$ & $118.8(2)$ & $\mathrm{C}(12)-\mathrm{C}(4)-\mathrm{C}(5)$ & $119.7(2)$ \\
\hline $\mathrm{C}(3)-\mathrm{C}(4)-\mathrm{C}(5)$ & $121.39(19)$ & $\mathrm{C}(20)-\mathrm{C}(5)-\mathrm{C}(6)$ & $119.5(2)$ \\
\hline$C(20)-C(5)-C(4)$ & $121.13(19)$ & $C(6)-C(5)-C(4)$ & $119.3(2)$ \\
\hline$C(16)-C(6)-C(5)$ & $119.2(2)$ & $\mathrm{C}(16)-\mathrm{C}(6)-\mathrm{C}(7)$ & $122.26(19)$ \\
\hline$C(5)-C(6)-C(7)$ & $118.51(19)$ & $\mathrm{N}(1)-\mathrm{C}(7)-\mathrm{C}(6)$ & $113.12(18)$ \\
\hline
\end{tabular}




\begin{tabular}{|c|c|c|c|}
\hline $\mathrm{N}(1)-\mathrm{C}(7)-\mathrm{C}(14)$ & $109.00(18)$ & $C(6)-C(7)-C(14)$ & $111.55(18)$ \\
\hline $\mathrm{N}(1)-\mathrm{C}(7)-\mathrm{H}(7)$ & 107.6 & $\mathrm{C}(6)-\mathrm{C}(7)-\mathrm{H}(7)$ & 107.6 \\
\hline $\mathrm{C}(14)-\mathrm{C}(7)-\mathrm{H}(7)$ & 107.6 & $\mathrm{O}(2)-\mathrm{C}(8)-\mathrm{N}(1)$ & $121.6(2)$ \\
\hline $\mathrm{O}(2)-\mathrm{C}(8)-\mathrm{C}(15)$ & $122.7(2)$ & $\mathrm{N}(1)-\mathrm{C}(8)-\mathrm{C}(15)$ & $115.8(2)$ \\
\hline $\mathrm{O}(3)-\mathrm{C}(9)-\mathrm{H}(9 \mathrm{~A})$ & 109.5 & $\mathrm{O}(3)-\mathrm{C}(9)-\mathrm{H}(9 \mathrm{~B})$ & 109.5 \\
\hline $\mathrm{H}(9 \mathrm{~A})-\mathrm{C}(9)-\mathrm{H}(9 \mathrm{~B})$ & 109.5 & $\mathrm{O}(3)-\mathrm{C}(9)-\mathrm{H}(9 \mathrm{C})$ & 109.5 \\
\hline $\mathrm{H}(9 \mathrm{~A})-\mathrm{C}(9)-\mathrm{H}(9 \mathrm{C})$ & 109.5 & $\mathrm{H}(9 \mathrm{~B})-\mathrm{C}(9)-\mathrm{H}(9 \mathrm{C})$ & 109.5 \\
\hline $\mathrm{O}(3)-\mathrm{C}(10)-\mathrm{C}(21)$ & $125.1(2)$ & $\mathrm{O}(3)-\mathrm{C}(10)-\mathrm{C}(2)$ & $114.6(2)$ \\
\hline $\mathrm{C}(21)-\mathrm{C}(10)-\mathrm{C}(2)$ & $120.20(19)$ & $\mathrm{O}(4)-\mathrm{C}(11)-\mathrm{H}(11 \mathrm{~A})$ & 109.5 \\
\hline $\mathrm{O}(4)-\mathrm{C}(11)-\mathrm{H}(11 \mathrm{~B})$ & 109.5 & $\mathrm{H}(11 \mathrm{~A})-\mathrm{C}(11)-\mathrm{H}(11 \mathrm{~B})$ & 109.5 \\
\hline $\mathrm{O}(4)-\mathrm{C}(11)-\mathrm{H}(11 \mathrm{C})$ & 109.5 & $\mathrm{H}(11 \mathrm{~A})-\mathrm{C}(11)-\mathrm{H}(11 \mathrm{C})$ & 109.5 \\
\hline $\mathrm{H}(11 \mathrm{~B})-\mathrm{C}(11)-\mathrm{H}(11 \mathrm{C})$ & 109.5 & $\mathrm{C}(21)-\mathrm{C}(12)-\mathrm{C}(4)$ & $120.6(2)$ \\
\hline $\mathrm{C}(21)-\mathrm{C}(12)-\mathrm{C}(13)$ & $120.4(2)$ & $\mathrm{C}(4)-\mathrm{C}(12)-\mathrm{C}(13)$ & $118.9(2)$ \\
\hline $\mathrm{C}(12)-\mathrm{C}(13)-\mathrm{C}(14)$ & $112.00(18)$ & $\mathrm{C}(12)-\mathrm{C}(13)-\mathrm{H}(13 \mathrm{~A})$ & 109.2 \\
\hline $\mathrm{C}(14)-\mathrm{C}(13)-\mathrm{H}(13 \mathrm{~A})$ & 109.2 & $\mathrm{C}(12)-\mathrm{C}(13)-\mathrm{H}(13 \mathrm{~B})$ & 109.2 \\
\hline $\mathrm{C}(14)-\mathrm{C}(13)-\mathrm{H}(13 \mathrm{~B})$ & 109.2 & $\mathrm{H}(13 \mathrm{~A})-\mathrm{C}(13)-\mathrm{H}(13 \mathrm{~B})$ & 107.9 \\
\hline $\mathrm{C}(7)-\mathrm{C}(14)-\mathrm{C}(13)$ & $113.75(18)$ & $\mathrm{C}(7)-\mathrm{C}(14)-\mathrm{H}(14 \mathrm{~A})$ & 108.8 \\
\hline $\mathrm{C}(13)-\mathrm{C}(14)-\mathrm{H}(14 \mathrm{~A})$ & 108.8 & $\mathrm{C}(7)-\mathrm{C}(14)-\mathrm{H}(14 \mathrm{~B})$ & 108.8 \\
\hline $\mathrm{C}(13)-\mathrm{C}(14)-\mathrm{H}(14 \mathrm{~B})$ & 108.8 & $\mathrm{H}(14 \mathrm{~A})-\mathrm{C}(14)-\mathrm{H}(14 \mathrm{~B})$ & 107.7 \\
\hline $\mathrm{C}(8)-\mathrm{C}(15)-\mathrm{H}(15 \mathrm{~A})$ & 109.5 & $\mathrm{C}(8)-\mathrm{C}(15)-\mathrm{H}(15 \mathrm{~B})$ & 109.5 \\
\hline $\mathrm{H}(15 \mathrm{~A})-\mathrm{C}(15)-\mathrm{H}(15 \mathrm{~B})$ & 109.5 & $\mathrm{C}(8)-\mathrm{C}(15)-\mathrm{H}(15 \mathrm{C})$ & 109.5 \\
\hline $\mathrm{H}(15 \mathrm{~A})-\mathrm{C}(15)-\mathrm{H}(15 \mathrm{C})$ & 109.5 & $\mathrm{H}(15 \mathrm{~B})-\mathrm{C}(15)-\mathrm{H}(15 \mathrm{C})$ & 109.5 \\
\hline$C(17)-C(16)-C(6)$ & $121.3(2)$ & $\mathrm{C}(17)-\mathrm{C}(16)-\mathrm{H}(16)$ & 119.3 \\
\hline $\mathrm{C}(6)-\mathrm{C}(16)-\mathrm{H}(16)$ & 119.3 & $\mathrm{O}(5)-\mathrm{C}(17)-\mathrm{C}(16)$ & $119.0(2)$ \\
\hline $\mathrm{O}(5)-\mathrm{C}(17)-\mathrm{C}(18)$ & $121.5(2)$ & $C(16)-C(17)-C(18)$ & $119.5(2)$ \\
\hline $\mathrm{O}(6)-\mathrm{C}(18)-\mathrm{C}(20)$ & $125.2(2)$ & $\mathrm{O}(6)-\mathrm{C}(18)-\mathrm{C}(17)$ & $115.0(2)$ \\
\hline$C(20)-C(18)-C(17)$ & $119.8(2)$ & $\mathrm{O}(6)-\mathrm{C}(19)-\mathrm{H}(19 \mathrm{~A})$ & 109.5 \\
\hline $\mathrm{O}(6)-\mathrm{C}(19)-\mathrm{H}(19 \mathrm{~B})$ & 109.5 & $\mathrm{H}(19 \mathrm{~A})-\mathrm{C}(19)-\mathrm{H}(19 \mathrm{~B})$ & 109.5 \\
\hline $\mathrm{O}(6)-\mathrm{C}(19)-\mathrm{H}(19 \mathrm{C})$ & 109.5 & $\mathrm{H}(19 \mathrm{~A})-\mathrm{C}(19)-\mathrm{H}(19 \mathrm{C})$ & 109.5 \\
\hline
\end{tabular}




\begin{tabular}{llll}
\hline $\mathrm{H}(19 \mathrm{~B})-\mathrm{C}(19)-\mathrm{H}(19 \mathrm{C})$ & 109.5 & $\mathrm{C}(18)-\mathrm{C}(20)-\mathrm{C}(5)$ & $120.5(2)$ \\
$\mathrm{C}(18)-\mathrm{C}(20)-\mathrm{H}(20)$ & 119.7 & $\mathrm{C}(5)-\mathrm{C}(20)-\mathrm{H}(20)$ & 119.7 \\
$\mathrm{C}(10)-\mathrm{C}(21)-\mathrm{C}(12)$ & $120.0(2)$ & $\mathrm{C}(10)-\mathrm{C}(21)-\mathrm{H}(21)$ & 120.0 \\
$\mathrm{C}(12)-\mathrm{C}(21)-\mathrm{H}(21)$ & 120.0 & $\mathrm{C}(23)-\mathrm{C}(22)-\mathrm{H}(22 \mathrm{~A})$ & 109.5 \\
$\mathrm{C}(23)-\mathrm{C}(22)-\mathrm{H}(22 \mathrm{~B})$ & 109.5 & $\mathrm{H}(22 \mathrm{~A})-\mathrm{C}(22)-\mathrm{H}(22 \mathrm{~B})$ & 109.5 \\
$\mathrm{C}(23)-\mathrm{C}(22)-\mathrm{H}(22 \mathrm{C})$ & 109.5 & $\mathrm{H}(22 \mathrm{~A})-\mathrm{C}(22)-\mathrm{H}(22 \mathrm{C})$ & 109.5 \\
$\mathrm{H}(22 \mathrm{~B})-\mathrm{C}(22)-\mathrm{H}(22 \mathrm{C})$ & 109.5 & $\mathrm{O}(7)-\mathrm{C}(23)-\mathrm{C}(22)$ & $120.0(4)$ \\
$\mathrm{O}(7)-\mathrm{C}(23)-\mathrm{C}(24)$ & $121.9(4)$ & $\mathrm{C}(22)-\mathrm{C}(23)-\mathrm{C}(24)$ & $118.1(3)$ \\
$\mathrm{C}(23)-\mathrm{C}(24)-\mathrm{H}(24 \mathrm{~A})$ & 109.5 & $\mathrm{C}(23)-\mathrm{C}(24)-\mathrm{H}(24 \mathrm{~B})$ & 109.5 \\
$\mathrm{H}(24 \mathrm{~A})-\mathrm{C}(24)-\mathrm{H}(24 \mathrm{~B})$ & 109.5 & $\mathrm{C}(23)-\mathrm{C}(24)-\mathrm{H}(24 \mathrm{C})$ & 109.5 \\
$\mathrm{H}(24 \mathrm{~A})-\mathrm{C}(24)-\mathrm{H}(24 \mathrm{C})$ & 109.5 & $\mathrm{H}(24 \mathrm{~B})-\mathrm{C}(24)-\mathrm{H}(24 \mathrm{C})$ & 109.5 \\
\hline
\end{tabular}

Table 4. Anisotropic Displacement Parameters $\left(\AA^{2} \times 10^{3}\right)$ for Compound 17. The anisotropic displacement factor exponent takes the form: $-2 \pi^{2}\left[h^{2} a^{* 2} U^{11}+\ldots+2 h k a^{*} b^{*} U^{12}\right]$.

\begin{tabular}{lcccccc}
\hline & $\mathrm{U}^{11}$ & $\mathrm{U}^{22}$ & $\mathrm{U}^{33}$ & $\mathrm{U}^{23}$ & $\mathrm{U}^{13}$ & $\mathrm{U}^{12}$ \\
\hline $\mathrm{N}(1)$ & $31(1)$ & $19(1)$ & $15(1)$ & $-4(1)$ & $1(1)$ & $-3(1)$ \\
$\mathrm{O}(2)$ & $37(1)$ & $22(1)$ & $41(1)$ & $-6(1)$ & $19(1)$ & $-6(1)$ \\
$\mathrm{O}(3)$ & $21(1)$ & $30(1)$ & $29(1)$ & $-2(1)$ & $3(1)$ & $5(1)$ \\
$\mathrm{O}(4)$ & $21(1)$ & $26(1)$ & $18(1)$ & $-2(1)$ & $2(1)$ & $-3(1)$ \\
$\mathrm{O}(5)$ & $31(1)$ & $43(1)$ & $23(1)$ & $-1(1)$ & $7(1)$ & $15(1)$ \\
$\mathrm{O}(6)$ & $25(1)$ & $39(1)$ & $21(1)$ & $-3(1)$ & $0(1)$ & $10(1)$ \\
$\mathrm{O}(7)$ & $91(2)$ & $124(3)$ & $133(3)$ & $-76(3)$ & $-67(2)$ & $56(2)$ \\
$\mathrm{O}(1)$ & $32(1)$ & $23(1)$ & $17(1)$ & $-1(1)$ & $2(1)$ & $5(1)$ \\
$\mathrm{C}(1)$ & $55(2)$ & $34(2)$ & $20(1)$ & $-5(1)$ & $-1(1)$ & $2(1)$ \\
$\mathrm{C}(2)$ & $27(1)$ & $19(1)$ & $14(1)$ & $3(1)$ & $2(1)$ & $1(1)$ \\
$\mathrm{C}(3)$ & $22(1)$ & $22(1)$ & $10(1)$ & $4(1)$ & $1(1)$ & $0(1)$ \\
$\mathrm{C}(4)$ & $22(1)$ & $21(1)$ & $14(1)$ & $2(1)$ & $3(1)$ & $1(1)$ \\
$\mathrm{C}(5)$ & $23(1)$ & $18(1)$ & $18(1)$ & $0(1)$ & $3(1)$ & $-2(1)$ \\
\hline
\end{tabular}




\begin{tabular}{lllllll}
\hline $\mathrm{C}(6)$ & $24(1)$ & $15(1)$ & $18(1)$ & $0(1)$ & $3(1)$ & $-2(1)$ \\
$\mathrm{C}(7)$ & $28(1)$ & $19(1)$ & $14(1)$ & $-1(1)$ & $2(1)$ & $-2(1)$ \\
$\mathrm{C}(8)$ & $18(1)$ & $23(1)$ & $22(1)$ & $1(1)$ & $2(1)$ & $2(1)$ \\
$\mathrm{C}(9)$ & $20(1)$ & $44(2)$ & $42(2)$ & $-6(1)$ & $4(1)$ & $6(1)$ \\
$\mathrm{C}(10)$ & $24(1)$ & $26(1)$ & $16(1)$ & $6(1)$ & $3(1)$ & $4(1)$ \\
$\mathrm{C}(11)$ & $21(1)$ & $26(1)$ & $29(1)$ & $5(1)$ & $3(1)$ & $-3(1)$ \\
$\mathrm{C}(12)$ & $26(1)$ & $22(1)$ & $13(1)$ & $5(1)$ & $2(1)$ & $-2(1)$ \\
$\mathrm{C}(13)$ & $24(1)$ & $22(1)$ & $22(1)$ & $1(1)$ & $4(1)$ & $-5(1)$ \\
$\mathrm{C}(14)$ & $26(1)$ & $23(1)$ & $21(1)$ & $-3(1)$ & $2(1)$ & $-5(1)$ \\
$\mathrm{C}(15)$ & $39(1)$ & $32(1)$ & $25(1)$ & $5(1)$ & $9(1)$ & $4(1)$ \\
$\mathrm{C}(16)$ & $32(1)$ & $21(1)$ & $16(1)$ & $-2(1)$ & $6(1)$ & $1(1)$ \\
$\mathrm{C}(17)$ & $26(1)$ & $21(1)$ & $21(1)$ & $-2(1)$ & $7(1)$ & $2(1)$ \\
$\mathrm{C}(18)$ & $23(1)$ & $24(1)$ & $20(1)$ & $1(1)$ & $2(1)$ & $2(1)$ \\
$\mathrm{C}(19)$ & $38(1)$ & $44(2)$ & $21(1)$ & $0(1)$ & $-3(1)$ & $14(1)$ \\
$\mathrm{C}(20)$ & $25(1)$ & $20(1)$ & $16(1)$ & $-1(1)$ & $4(1)$ & $2(1)$ \\
$\mathrm{C}(21)$ & $20(1)$ & $25(1)$ & $18(1)$ & $6(1)$ & $1(1)$ & $-2(1)$ \\
$\mathrm{C}(22)$ & $90(3)$ & $49(2)$ & $44(2)$ & $2(2)$ & $9(2)$ & $-16(2)$ \\
$\mathrm{C}(23)$ & $68(2)$ & $28(2)$ & $62(2)$ & $-14(1)$ & $-21(2)$ & $14(2)$ \\
$\mathrm{C}(24)$ & $71(2)$ & $35(2)$ & $65(2)$ & $-10(2)$ & $11(2)$ & $11(2)$ \\
\hline & & & & & \\
\hline
\end{tabular}

Table 5. Hydrogen Coordinates $\left(\times 10^{4}\right)$ and Isotropic Displacement Parameters $\left(\AA^{2} \mathrm{x} 10^{3}\right)$ for Compound 17.

\begin{tabular}{lcccc}
\hline & $\mathrm{x}$ & $\mathrm{y}$ & $\mathrm{z}$ & $\mathrm{U}_{\text {eq }}$ \\
\hline $\mathrm{H}(1)$ & 1660 & -964 & 2722 & 26 \\
$\mathrm{H}(5)$ & 6344 & -2151 & 6223 & 48 \\
$\mathrm{H}(1 \mathrm{~A})$ & -580 & 2884 & 10431 & 54 \\
$\mathrm{H}(1 \mathrm{~B})$ & 191 & 4133 & 10330 & 54 \\
$\mathrm{H}(1 \mathrm{C})$ & 1010 & 2905 & 10458 & 54 \\
\hline
\end{tabular}




\begin{tabular}{|c|c|c|c|c|}
\hline $\mathrm{H}(7)$ & 1080 & 552 & 4724 & 24 \\
\hline $\mathrm{H}(9 \mathrm{~A})$ & -3597 & 1487 & 6958 & 53 \\
\hline $\mathrm{H}(9 \mathrm{~B})$ & -4186 & 2164 & 8179 & 53 \\
\hline $\mathrm{H}(9 \mathrm{C})$ & -3569 & 848 & 8364 & 53 \\
\hline $\mathrm{H}(11 \mathrm{~A})$ & 3422 & 1898 & 6565 & 38 \\
\hline $\mathrm{H}(11 \mathrm{~B})$ & 3740 & 3104 & 7374 & 38 \\
\hline $\mathrm{H}(11 \mathrm{C})$ & 2356 & 2964 & 6536 & 38 \\
\hline $\mathrm{H}(13 \mathrm{~A})$ & -967 & -1694 & 6171 & 27 \\
\hline $\mathrm{H}(13 \mathrm{~B})$ & 574 & -1919 & 6566 & 27 \\
\hline $\mathrm{H}(14 \mathrm{~A})$ & 502 & -1941 & 4329 & 28 \\
\hline $\mathrm{H}(14 \mathrm{~B})$ & -537 & -857 & 4205 & 28 \\
\hline $\mathrm{H}(15 \mathrm{~A})$ & 1905 & 921 & 602 & 47 \\
\hline $\mathrm{H}(15 \mathrm{~B})$ & 2563 & -352 & 962 & 47 \\
\hline $\mathrm{H}(15 \mathrm{C})$ & 3453 & 838 & 1042 & 47 \\
\hline $\mathrm{H}(16)$ & 3852 & -1206 & 4211 & 27 \\
\hline $\mathrm{H}(19 \mathrm{~A})$ & 5348 & -667 & 9866 & 51 \\
\hline $\mathrm{H}(19 \mathrm{~B})$ & 6297 & -1818 & 9990 & 51 \\
\hline $\mathrm{H}(19 \mathrm{C})$ & 4736 & -1982 & 9655 & 51 \\
\hline $\mathrm{H}(20)$ & 3424 & -343 & 8647 & 25 \\
\hline $\mathrm{H}(21)$ & -2021 & 8 & 7048 & 25 \\
\hline $\mathrm{H}(22 \mathrm{~A})$ & 8471 & -35 & 1955 & 91 \\
\hline $\mathrm{H}(22 \mathrm{~B})$ & 8723 & 1355 & 2272 & 91 \\
\hline $\mathrm{H}(22 \mathrm{C})$ & 8021 & 961 & 891 & 91 \\
\hline $\mathrm{H}(24 \mathrm{~A})$ & 5826 & 917 & 4104 & 85 \\
\hline $\mathrm{H}(24 \mathrm{~B})$ & 7237 & 1579 & 4216 & 85 \\
\hline $\mathrm{H}(24 \mathrm{C})$ & 7166 & 145 & 4235 & 85 \\
\hline
\end{tabular}

Table 6. Torsion Angles $\left[{ }^{\circ}\right]$ for Compound 17. 


\begin{tabular}{|c|c|c|c|}
\hline $\mathrm{C}(1)-\mathrm{O}(1)-\mathrm{C}(2)-\mathrm{C}(3)$ & $-95.3(2)$ & $\mathrm{C}(1)-\mathrm{O}(1)-\mathrm{C}(2)-\mathrm{C}(10)$ & $92.3(3)$ \\
\hline $\mathrm{C}(11)-\mathrm{O}(4)-\mathrm{C}(3)-\mathrm{C}(2)$ & $-105.9(2)$ & $\mathrm{C}(11)-\mathrm{O}(4)-\mathrm{C}(3)-\mathrm{C}(4)$ & $72.8(3)$ \\
\hline $\mathrm{O}(1)-\mathrm{C}(2)-\mathrm{C}(3)-\mathrm{O}(4)$ & $7.3(3)$ & $\mathrm{C}(10)-\mathrm{C}(2)-\mathrm{C}(3)-\mathrm{O}(4)$ & $179.64(18)$ \\
\hline $\mathrm{O}(1)-\mathrm{C}(2)-\mathrm{C}(3)-\mathrm{C}(4)$ & $-171.42(19)$ & $\mathrm{C}(10)-\mathrm{C}(2)-\mathrm{C}(3)-\mathrm{C}(4)$ & $0.9(3)$ \\
\hline $\mathrm{O}(4)-\mathrm{C}(3)-\mathrm{C}(4)-\mathrm{C}(12)$ & $-178.22(19)$ & $\mathrm{C}(2)-\mathrm{C}(3)-\mathrm{C}(4)-\mathrm{C}(12)$ & $0.4(3)$ \\
\hline $\mathrm{O}(4)-\mathrm{C}(3)-\mathrm{C}(4)-\mathrm{C}(5)$ & $-1.6(3)$ & $\mathrm{C}(2)-\mathrm{C}(3)-\mathrm{C}(4)-\mathrm{C}(5)$ & 177.09(19) \\
\hline $\mathrm{C}(12)-\mathrm{C}(4)-\mathrm{C}(5)-\mathrm{C}(20)$ & $-125.8(2)$ & $\mathrm{C}(3)-\mathrm{C}(4)-\mathrm{C}(5)-\mathrm{C}(20)$ & $57.6(3)$ \\
\hline $\mathrm{C}(12)-\mathrm{C}(4)-\mathrm{C}(5)-\mathrm{C}(6)$ & $54.9(3)$ & $\mathrm{C}(3)-\mathrm{C}(4)-\mathrm{C}(5)-\mathrm{C}(6)$ & $-121.7(2)$ \\
\hline $\mathrm{C}(20)-\mathrm{C}(5)-\mathrm{C}(6)-\mathrm{C}(16)$ & $-1.6(3)$ & $\mathrm{C}(4)-\mathrm{C}(5)-\mathrm{C}(6)-\mathrm{C}(16)$ & $177.7(2)$ \\
\hline $\mathrm{C}(20)-\mathrm{C}(5)-\mathrm{C}(6)-\mathrm{C}(7)$ & $177.0(2)$ & $\mathrm{C}(4)-\mathrm{C}(5)-\mathrm{C}(6)-\mathrm{C}(7)$ & $-3.6(3)$ \\
\hline $\mathrm{C}(8)-\mathrm{N}(1)-\mathrm{C}(7)-\mathrm{C}(6)$ & $-85.9(3)$ & $\mathrm{C}(8)-\mathrm{N}(1)-\mathrm{C}(7)-\mathrm{C}(14)$ & $149.3(2)$ \\
\hline $\mathrm{C}(16)-\mathrm{C}(6)-\mathrm{C}(7)-\mathrm{N}(1)$ & $-16.5(3)$ & $\mathrm{C}(5)-\mathrm{C}(6)-\mathrm{C}(7)-\mathrm{N}(1)$ & $164.95(19)$ \\
\hline$C(16)-C(6)-C(7)-C(14)$ & $106.9(2)$ & $\mathrm{C}(5)-\mathrm{C}(6)-\mathrm{C}(7)-\mathrm{C}(14)$ & $-71.7(2)$ \\
\hline $\mathrm{C}(7)-\mathrm{N}(1)-\mathrm{C}(8)-\mathrm{O}(2)$ & $-0.3(3)$ & $\mathrm{C}(7)-\mathrm{N}(1)-\mathrm{C}(8)-\mathrm{C}(15)$ & $179.8(2)$ \\
\hline $\mathrm{C}(9)-\mathrm{O}(3)-\mathrm{C}(10)-\mathrm{C}(21)$ & $1.5(3)$ & $\mathrm{C}(9)-\mathrm{O}(3)-\mathrm{C}(10)-\mathrm{C}(2)$ & $178.5(2)$ \\
\hline $\mathrm{O}(1)-\mathrm{C}(2)-\mathrm{C}(10)-\mathrm{O}(3)$ & $-5.9(3)$ & $\mathrm{C}(3)-\mathrm{C}(2)-\mathrm{C}(10)-\mathrm{O}(3)$ & $-178.31(19)$ \\
\hline $\mathrm{O}(1)-\mathrm{C}(2)-\mathrm{C}(10)-\mathrm{C}(21)$ & $171.27(19)$ & $\mathrm{C}(3)-\mathrm{C}(2)-\mathrm{C}(10)-\mathrm{C}(21)$ & $-1.1(3)$ \\
\hline $\mathrm{C}(3)-\mathrm{C}(4)-\mathrm{C}(12)-\mathrm{C}(21)$ & $-1.6(3)$ & $\mathrm{C}(5)-\mathrm{C}(4)-\mathrm{C}(12)-\mathrm{C}(21)$ & $-178.31(19)$ \\
\hline $\mathrm{C}(3)-\mathrm{C}(4)-\mathrm{C}(12)-\mathrm{C}(13)$ & 176.63(19) & $\mathrm{C}(5)-\mathrm{C}(4)-\mathrm{C}(12)-\mathrm{C}(13)$ & $-0.1(3)$ \\
\hline $\mathrm{C}(21)-\mathrm{C}(12)-\mathrm{C}(13)-\mathrm{C}(14)$ & $106.2(2)$ & $\mathrm{C}(4)-\mathrm{C}(12)-\mathrm{C}(13)-\mathrm{C}(14)$ & $-72.0(3)$ \\
\hline $\mathrm{N}(1)-\mathrm{C}(7)-\mathrm{C}(14)-\mathrm{C}(13)$ & 171.93(19) & $\mathrm{C}(6)-\mathrm{C}(7)-\mathrm{C}(14)-\mathrm{C}(13)$ & $46.3(3)$ \\
\hline $\mathrm{C}(12)-\mathrm{C}(13)-\mathrm{C}(14)-\mathrm{C}(7)$ & $40.6(3)$ & $\mathrm{C}(5)-\mathrm{C}(6)-\mathrm{C}(16)-\mathrm{C}(17)$ & $1.9(3)$ \\
\hline$C(7)-C(6)-C(16)-C(17)$ & $-176.7(2)$ & $\mathrm{C}(6)-\mathrm{C}(16)-\mathrm{C}(17)-\mathrm{O}(5)$ & $-180.0(2)$ \\
\hline $\mathrm{C}(6)-\mathrm{C}(16)-\mathrm{C}(17)-\mathrm{C}(18)$ & $-0.2(3)$ & $\mathrm{C}(19)-\mathrm{O}(6)-\mathrm{C}(18)-\mathrm{C}(20)$ & $17.5(3)$ \\
\hline $\mathrm{C}(19)-\mathrm{O}(6)-\mathrm{C}(18)-\mathrm{C}(17)$ & $-163.7(2)$ & $\mathrm{O}(5)-\mathrm{C}(17)-\mathrm{C}(18)-\mathrm{O}(6)$ & $-0.8(3)$ \\
\hline $\mathrm{C}(16)-\mathrm{C}(17)-\mathrm{C}(18)-\mathrm{O}(6)$ & $179.4(2)$ & $\mathrm{O}(5)-\mathrm{C}(17)-\mathrm{C}(18)-\mathrm{C}(20)$ & $178.0(2)$ \\
\hline $\mathrm{C}(16)-\mathrm{C}(17)-\mathrm{C}(18)-\mathrm{C}(20)$ & $-1.7(3)$ & $\mathrm{O}(6)-\mathrm{C}(18)-\mathrm{C}(20)-\mathrm{C}(5)$ & $-179.3(2)$ \\
\hline $\mathrm{C}(17)-\mathrm{C}(18)-\mathrm{C}(20)-\mathrm{C}(5)$ & $2.0(3)$ & $\mathrm{C}(6)-\mathrm{C}(5)-\mathrm{C}(20)-\mathrm{C}(18)$ & $-0.3(3)$ \\
\hline$C(4)-C(5)-C(20)-C(18)$ & $-179.6(2)$ & $\mathrm{O}(3)-\mathrm{C}(10)-\mathrm{C}(21)-\mathrm{C}(12)$ & $176.9(2)$ \\
\hline
\end{tabular}




\begin{tabular}{llll}
\hline $\mathrm{C}(2)-\mathrm{C}(10)-\mathrm{C}(21)-\mathrm{C}(12)$ & $0.0(3)$ & $\mathrm{C}(4)-\mathrm{C}(12)-\mathrm{C}(21)-\mathrm{C}(10)$ & $1.4(3)$ \\
$\mathrm{C}(13)-\mathrm{C}(12)-\mathrm{C}(21)-\mathrm{C}(10)$ & $-176.8(2)$ & & \\
\hline
\end{tabular}

Table 7. Hydrogen Bonds for Compound 17 ( $\AA^{\text {and }}{ }^{\circ}$ ).

\begin{tabular}{|c|c|c|c|c|}
\hline D-H...A & $\mathrm{d}(\mathrm{D}-\mathrm{H})$ & $\mathrm{d}(\mathrm{H} \ldots \mathrm{A})$ & $\mathrm{d}(\mathrm{D} \ldots \mathrm{A})$ & $<(\mathrm{DHA})$ \\
\hline $\mathrm{C}(22)-\mathrm{H}(22 \mathrm{~A}) \ldots \mathrm{O}(1) \# 1$ & 0.98 & 2.55 & $3.450(5)$ & 153.3 \\
\hline $\mathrm{C}(19)-\mathrm{H}(19 \mathrm{~B}) \ldots \mathrm{O}(4) \# 2$ & 0.98 & 2.52 & $3.440(3)$ & 155.7 \\
\hline $\mathrm{C}(19)-\mathrm{H}(19 \mathrm{~A}) \ldots \mathrm{O}(7) \# 3$ & 0.98 & 2.56 & $3.426(4)$ & 148.0 \\
\hline $\mathrm{C}(15)-\mathrm{H}(15 \mathrm{C}) \ldots \mathrm{O}(7)$ & 0.98 & 2.43 & $3.303(4)$ & 148.1 \\
\hline $\mathrm{C}(9)-\mathrm{H}(9 \mathrm{C}) \ldots \mathrm{O}(6) \# 4$ & 0.98 & 2.61 & $3.466(4)$ & 146.2 \\
\hline $\mathrm{O}(5)-\mathrm{H}(5) \ldots \mathrm{O}(6)$ & 0.84 & 2.27 & $2.688(2)$ & 110.7 \\
\hline $\mathrm{O}(5)-\mathrm{H}(5) \ldots \mathrm{O}(2) \# 1$ & 0.84 & 1.93 & $2.681(2)$ & 148.4 \\
\hline $\mathrm{N}(1)-\mathrm{H}(1) \ldots \mathrm{O}(1) \# 5$ & 0.88 & 2.44 & $3.128(3)$ & 134.8 \\
\hline $\mathrm{N}(1)-\mathrm{H}(1) \ldots \mathrm{O}(3) \# 5$ & 0.88 & 2.42 & $3.253(3)$ & 158.9 \\
\hline $\mathrm{C}(22)-\mathrm{H}(22 \mathrm{~A}) \ldots \mathrm{O}(1) \# 1$ & 0.98 & 2.55 & $3.450(5)$ & 153.3 \\
\hline $\mathrm{C}(19)-\mathrm{H}(19 \mathrm{~B}) \ldots \mathrm{O}(4) \# 2$ & 0.98 & 2.52 & $3.440(3)$ & 155.7 \\
\hline $\mathrm{C}(19)-\mathrm{H}(19 \mathrm{~A}) \ldots \mathrm{O}(7) \# 3$ & 0.98 & 2.56 & $3.426(4)$ & 148.0 \\
\hline $\mathrm{C}(15)-\mathrm{H}(15 \mathrm{C}) \ldots \mathrm{O}(7)$ & 0.98 & 2.43 & $3.303(4)$ & 148.1 \\
\hline $\mathrm{C}(9)-\mathrm{H}(9 \mathrm{C}) \ldots \mathrm{O}(6) \# 4$ & 0.98 & 2.61 & $3.466(4)$ & 146.2 \\
\hline $\mathrm{O}(5)-\mathrm{H}(5) \ldots \mathrm{O}(6)$ & 0.84 & 2.27 & $2.688(2)$ & 110.7 \\
\hline $\mathrm{O}(5)-\mathrm{H}(5) \ldots \mathrm{O}(2) \# 1$ & 0.84 & 1.93 & $2.681(2)$ & 148.4 \\
\hline $\mathrm{N}(1)-\mathrm{H}(1) \ldots \mathrm{O}(1) \# 5$ & 0.88 & 2.44 & $3.128(3)$ & 134.8 \\
\hline $\mathrm{N}(1)-\mathrm{H}(1) \ldots \mathrm{O}(3) \# 5$ & 0.88 & 2.42 & $3.253(3)$ & 158.9 \\
\hline $\mathrm{C}(22)-\mathrm{H}(22 \mathrm{~A}) \ldots \mathrm{O}(1) \# 1$ & 0.98 & 2.55 & $3.450(5)$ & 153.3 \\
\hline $\mathrm{C}(19)-\mathrm{H}(19 \mathrm{~B}) \ldots \mathrm{O}(4) \# 2$ & 0.98 & 2.52 & $3.440(3)$ & 155.7 \\
\hline $\mathrm{C}(19)-\mathrm{H}(19 \mathrm{~A}) \ldots \mathrm{O}(7) \# 3$ & 0.98 & 2.56 & $3.426(4)$ & 148.0 \\
\hline $\mathrm{C}(15)-\mathrm{H}(15 \mathrm{C}) \ldots \mathrm{O}(7)$ & 0.98 & 2.43 & $3.303(4)$ & 148.1 \\
\hline $\mathrm{C}(9)-\mathrm{H}(9 \mathrm{C}) \ldots \mathrm{O}(6) \# 4$ & 0.98 & 2.61 & $3.466(4)$ & 146.2 \\
\hline
\end{tabular}




\begin{tabular}{|c|c|c|c|c|}
\hline $\mathrm{O}(5)-\mathrm{H}(5) \ldots \mathrm{O}(6)$ & 0.84 & 2.27 & $2.688(2)$ & 110.7 \\
\hline $\mathrm{O}(5)-\mathrm{H}(5) \ldots \mathrm{O}(2) \# 1$ & 0.84 & 1.93 & $2.681(2)$ & 148.4 \\
\hline $\mathrm{N}(1)-\mathrm{H}(1) \ldots \mathrm{O}(1) \# 5$ & 0.88 & 2.44 & $3.128(3)$ & 134.8 \\
\hline $\mathrm{N}(1)-\mathrm{H}(1) \ldots \mathrm{O}(3) \# 5$ & 0.88 & 2.42 & $3.253(3)$ & 158.9 \\
\hline $\mathrm{N}(1)-\mathrm{H}(1) \ldots \mathrm{O}(3) \# 5$ & 0.88 & 2.42 & $3.253(3)$ & 158.9 \\
\hline $\mathrm{N}(1)-\mathrm{H}(1) \ldots \mathrm{O}(1) \# 5$ & 0.88 & 2.44 & $3.128(3)$ & 134.8 \\
\hline $\mathrm{O}(5)-\mathrm{H}(5) \ldots \mathrm{O}(2) \# 1$ & 0.84 & 1.93 & $2.681(2)$ & 148.4 \\
\hline $\mathrm{O}(5)-\mathrm{H}(5) \ldots \mathrm{O}(6)$ & 0.84 & 2.27 & $2.688(2)$ & 110.7 \\
\hline $\mathrm{C}(9)-\mathrm{H}(9 \mathrm{C}) \ldots \mathrm{O}(6) \# 4$ & 0.98 & 2.61 & $3.466(4)$ & 146.2 \\
\hline $\mathrm{C}(15)-\mathrm{H}(15 \mathrm{C}) \ldots \mathrm{O}(7)$ & 0.98 & 2.43 & $3.303(4)$ & 148.1 \\
\hline $\mathrm{C}(19)-\mathrm{H}(19 \mathrm{~A}) \ldots \mathrm{O}(7) \# 3$ & 0.98 & 2.56 & $3.426(4)$ & 148.0 \\
\hline $\mathrm{C}(19)-\mathrm{H}(19 \mathrm{~B}) \ldots \mathrm{O}(4) \# 2$ & 0.98 & 2.52 & $3.440(3)$ & 155.7 \\
\hline $\mathrm{C}(22)-\mathrm{H}(22 \mathrm{~A}) \ldots \mathrm{O}(1) \# 1$ & 0.98 & 2.55 & $3.450(5)$ & 153.3 \\
\hline $\mathrm{N}(1)-\mathrm{H}(1) \ldots \mathrm{O}(3) \# 5$ & 0.88 & 2.42 & $3.253(3)$ & 158.9 \\
\hline $\mathrm{N}(1)-\mathrm{H}(1) \ldots \mathrm{O}(1) \# 5$ & 0.88 & 2.44 & $3.128(3)$ & 134.8 \\
\hline $\mathrm{O}(5)-\mathrm{H}(5) \ldots \mathrm{O}(2) \# 1$ & 0.84 & 1.93 & $2.681(2)$ & 148.4 \\
\hline $\mathrm{O}(5)-\mathrm{H}(5) \ldots \mathrm{O}(6)$ & 0.84 & 2.27 & $2.688(2)$ & 110.7 \\
\hline $\mathrm{C}(9)-\mathrm{H}(9 \mathrm{C}) \ldots \mathrm{O}(6) \# 4$ & 0.98 & 2.61 & $3.466(4)$ & 146.2 \\
\hline $\mathrm{C}(15)-\mathrm{H}(15 \mathrm{C}) \ldots \mathrm{O}(7)$ & 0.98 & 2.43 & $3.303(4)$ & 148.1 \\
\hline $\mathrm{C}(19)-\mathrm{H}(19 \mathrm{~A}) \ldots \mathrm{O}(7) \# 3$ & 0.98 & 2.56 & $3.426(4)$ & 148.0 \\
\hline $\mathrm{C}(19)-\mathrm{H}(19 \mathrm{~B}) \ldots \mathrm{O}(4) \# 2$ & 0.98 & 2.52 & $3.440(3)$ & 155.7 \\
\hline $\mathrm{C}(22)-\mathrm{H}(22 \mathrm{~A}) \ldots \mathrm{O}(1) \# 1$ & 0.98 & 2.55 & $3.450(5)$ & 153.3 \\
\hline $\mathrm{N}(1)-\mathrm{H}(1) \ldots \mathrm{O}(3) \# 5$ & 0.88 & 2.42 & $3.253(3)$ & 158.9 \\
\hline $\mathrm{N}(1)-\mathrm{H}(1) \ldots \mathrm{O}(1) \# 5$ & 0.88 & 2.44 & $3.128(3)$ & 134.8 \\
\hline $\mathrm{O}(5)-\mathrm{H}(5) \ldots \mathrm{O}(2) \# 1$ & 0.84 & 1.93 & $2.681(2)$ & 148.4 \\
\hline $\mathrm{O}(5)-\mathrm{H}(5) \ldots \mathrm{O}(6)$ & 0.84 & 2.27 & $2.688(2)$ & 110.7 \\
\hline $\mathrm{C}(9)-\mathrm{H}(9 \mathrm{C}) \ldots \mathrm{O}(6) \# 4$ & 0.98 & 2.61 & $3.466(4)$ & 146.2 \\
\hline $\mathrm{C}(15)-\mathrm{H}(15 \mathrm{C}) \ldots \mathrm{O}(7)$ & 0.98 & 2.43 & $3.303(4)$ & 148.1 \\
\hline $\mathrm{C}(19)-\mathrm{H}(19 \mathrm{~A}) \ldots \mathrm{O}(7) \# 3$ & 0.98 & 2.56 & $3.426(4)$ & 148.0 \\
\hline
\end{tabular}




\begin{tabular}{lllll}
\hline $\mathrm{C}(19)-\mathrm{H}(19 \mathrm{~B}) \ldots \mathrm{O}(4) \# 2$ & 0.98 & 2.52 & $3.440(3)$ & 155.7 \\
$\mathrm{C}(22)-\mathrm{H}(22 \mathrm{~A}) \ldots \mathrm{O}(1) \# 1$ & 0.98 & 2.55 & $3.450(5)$ & 153.3 \\
& & & & \\
\end{tabular}




\section{High Resolution Mass Spectra}<smiles>C=CC(O)c1ccc(OC)c(O)c1</smiles>

13

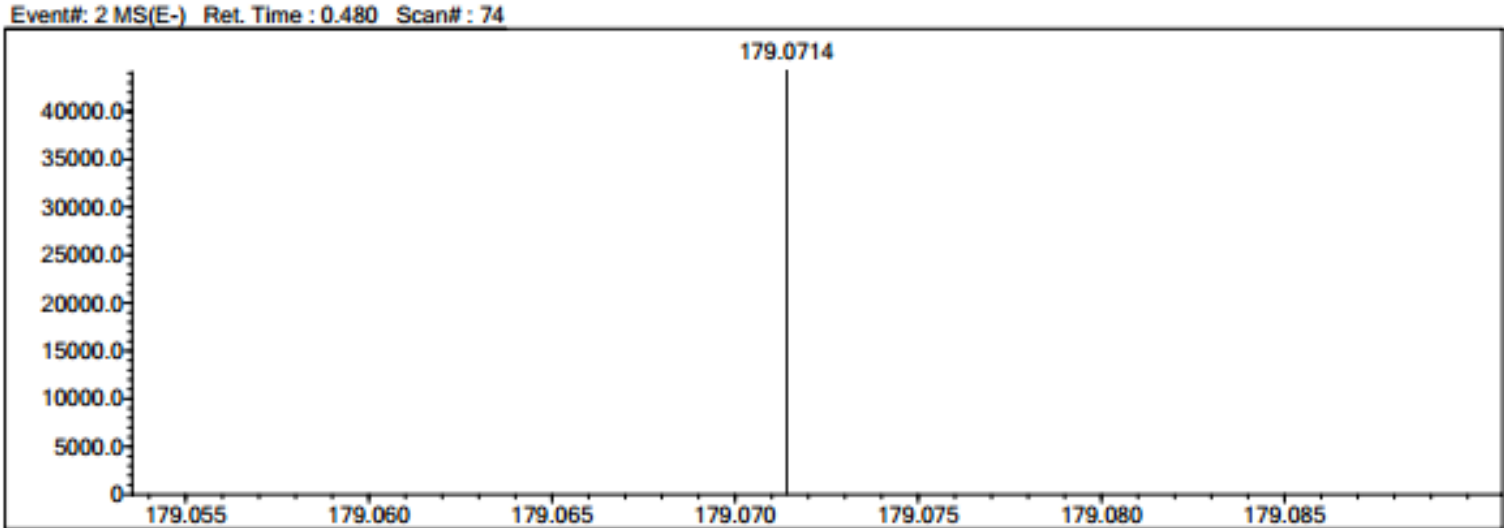

Measured region for $179.0714 \mathrm{~m} / \mathrm{z}$

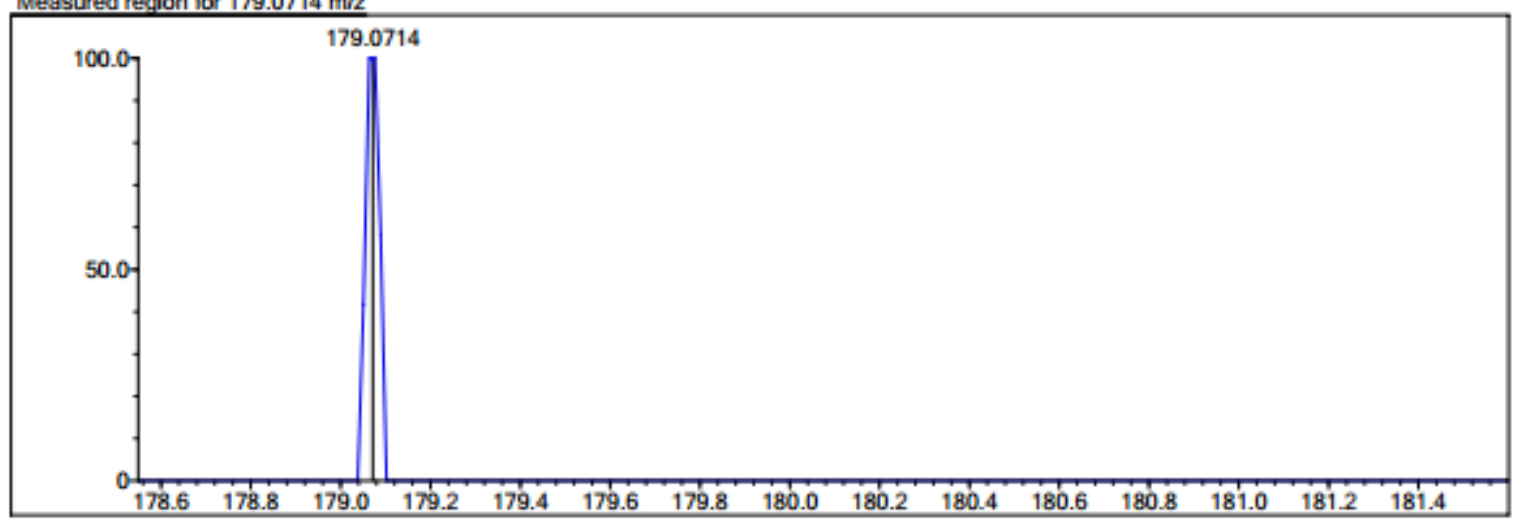

$\mathrm{C} 10 \mathrm{H} 12 \mathrm{O} 3[\mathrm{M}-\mathrm{H}]$ : : Predicted region for $179.0714 \mathrm{~m} / \mathrm{z}$

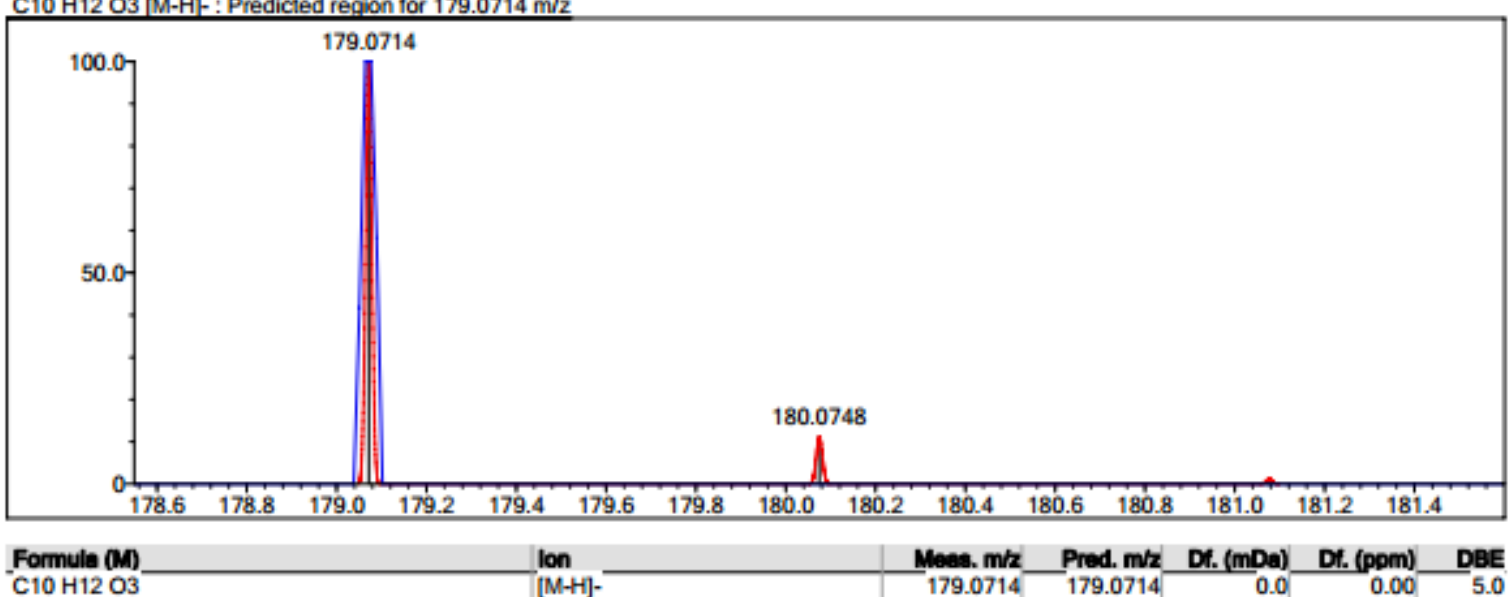


<smiles>C=C[C@H](N)c1ccc(OC)c(O)c1</smiles>

14
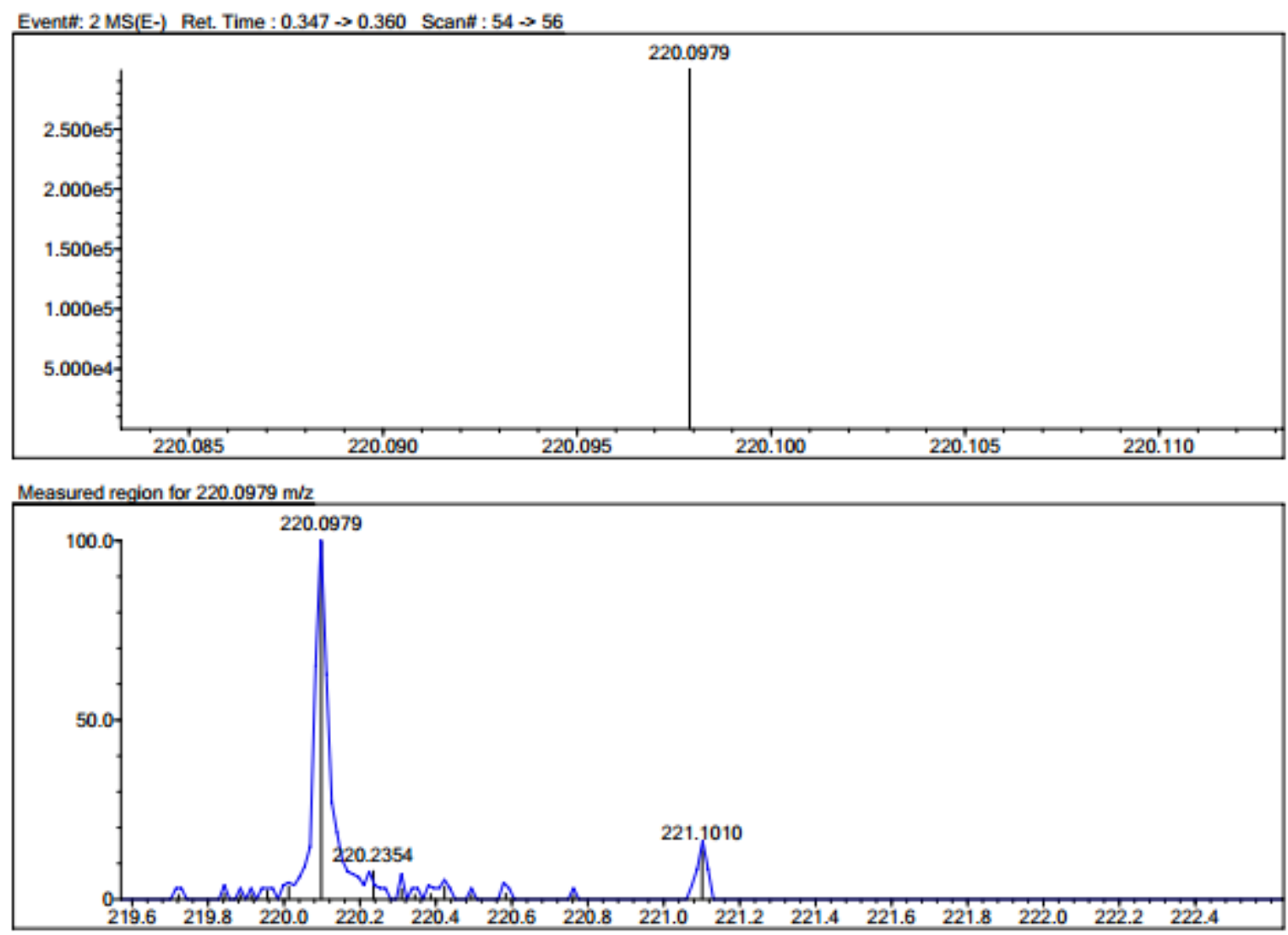

$\mathrm{C} 12 \mathrm{H} 15 \mathrm{~N} \mathrm{O3}[\mathrm{M}-\mathrm{H}]$-: Predicted region for $220.0979 \mathrm{~m} / 2$

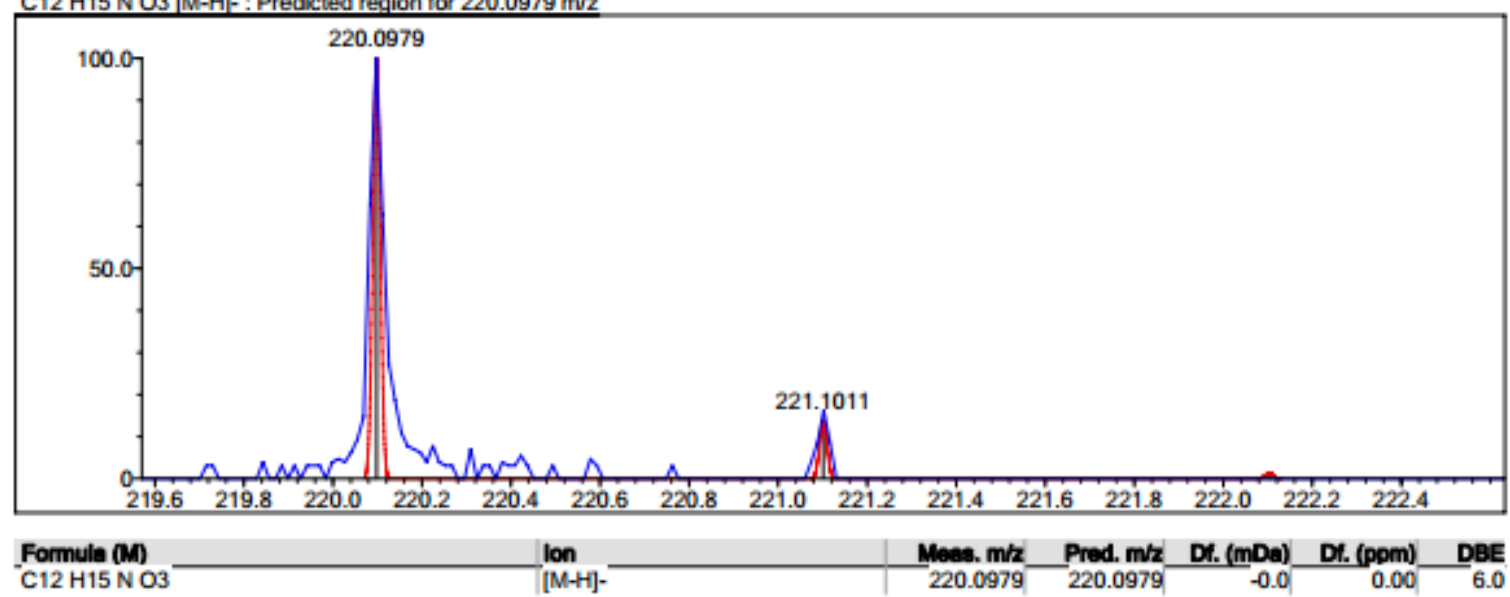


<smiles>CCCCCCCCN[C@@H](CCc1cc(OC)c(OC)c(OC)c1)c1ccc(OC)c(O)c1</smiles>
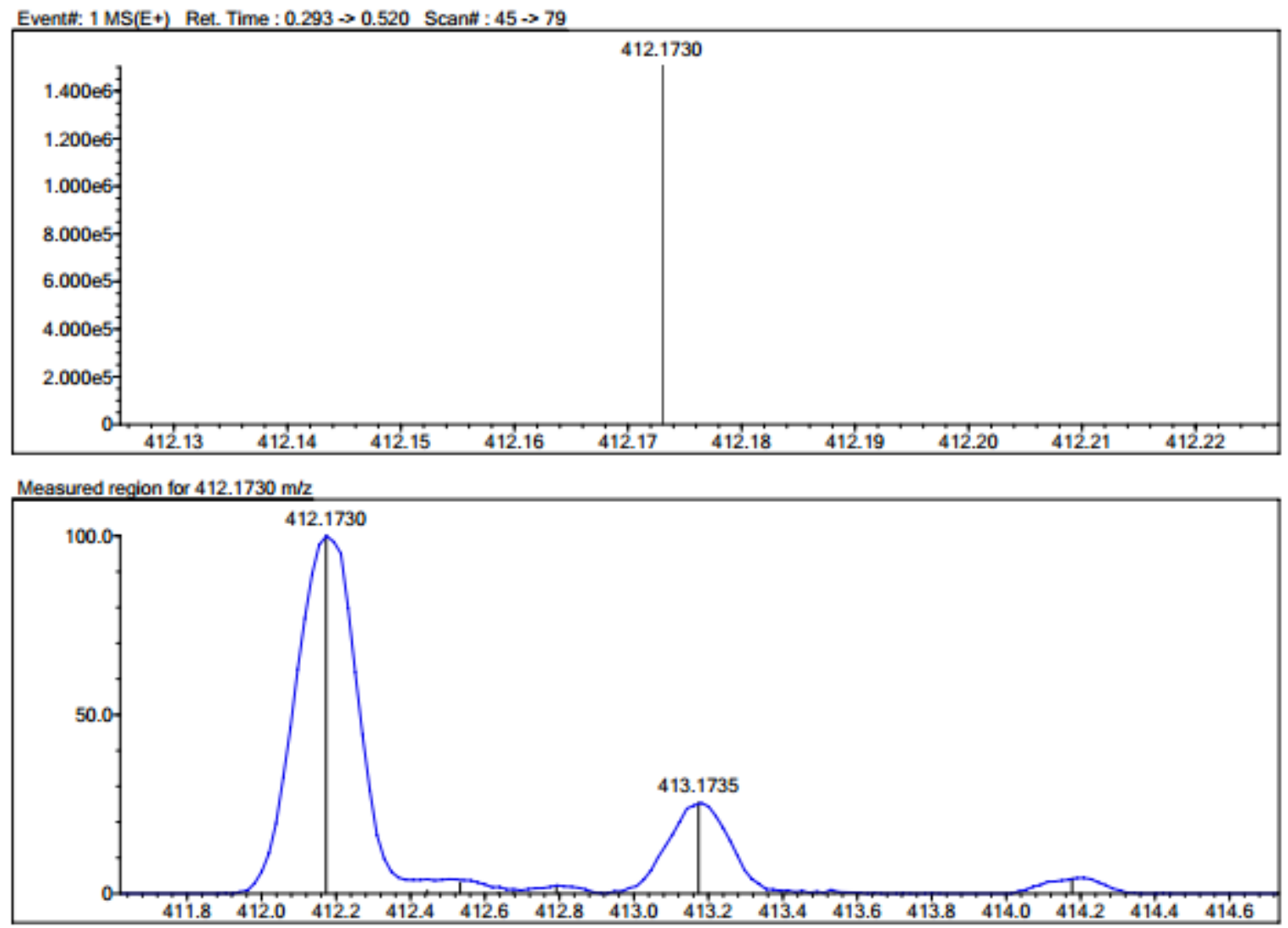

$\mathrm{C} 21 \mathrm{H} 27 \mathrm{~N} \mathrm{O6}[\mathrm{M}+\mathrm{Na}+\mathrm{t}$ : Predicted region for $412.1731 \mathrm{~m} / \mathrm{z}$

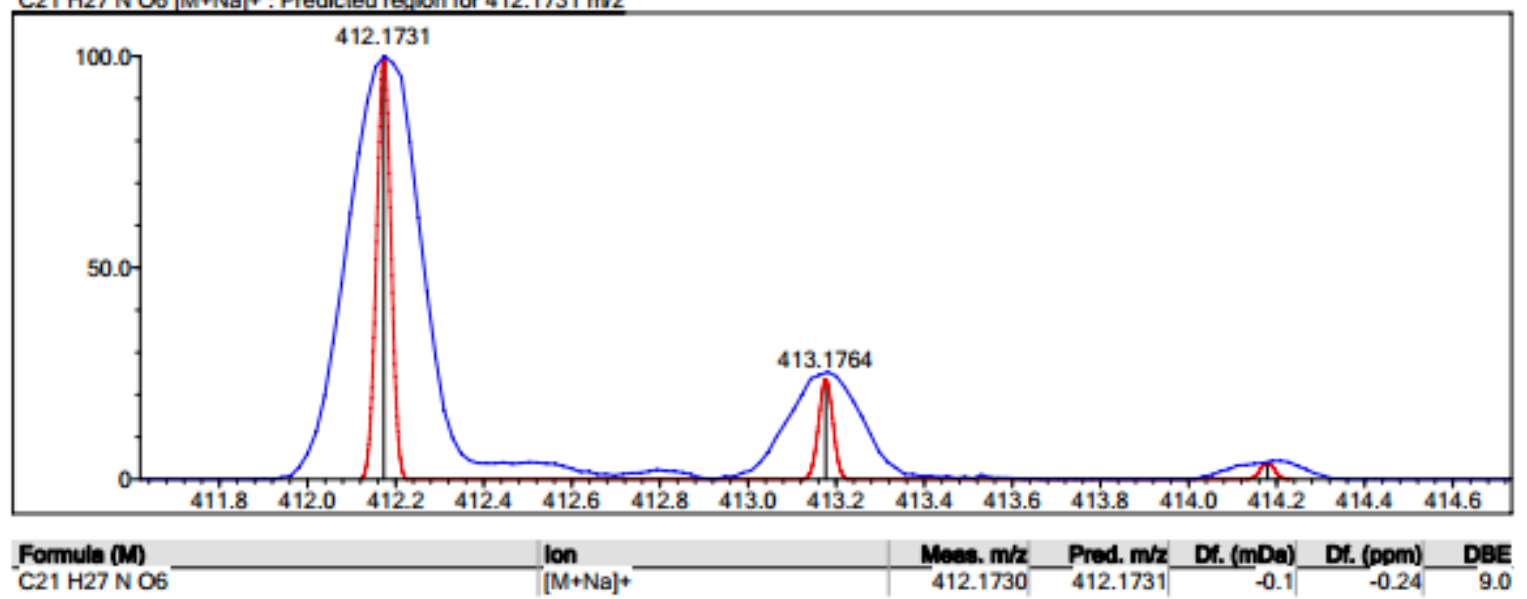


<smiles>CCCCCCNCCN[C@@H]1CCc2cc(OC)c(OC)c(OC)c2-c2cc(OC)c(O)cc21</smiles>
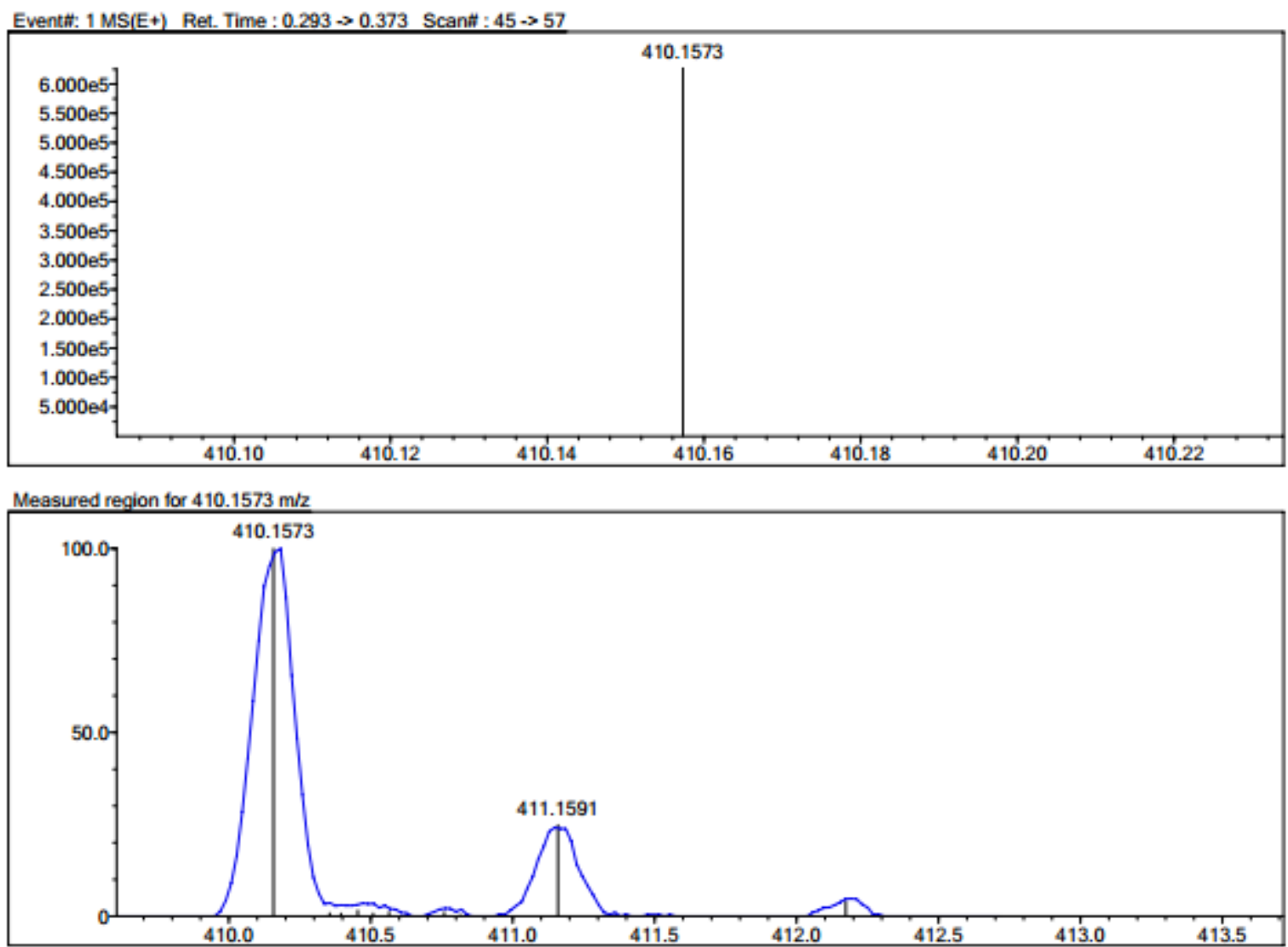

$\mathrm{C} 21 \mathrm{H} 25 \mathrm{~N} \mathrm{O6}[\mathrm{M}+\mathrm{Na}]+$ : Predicted region for $410.1574 \mathrm{~m} / \mathrm{z}$

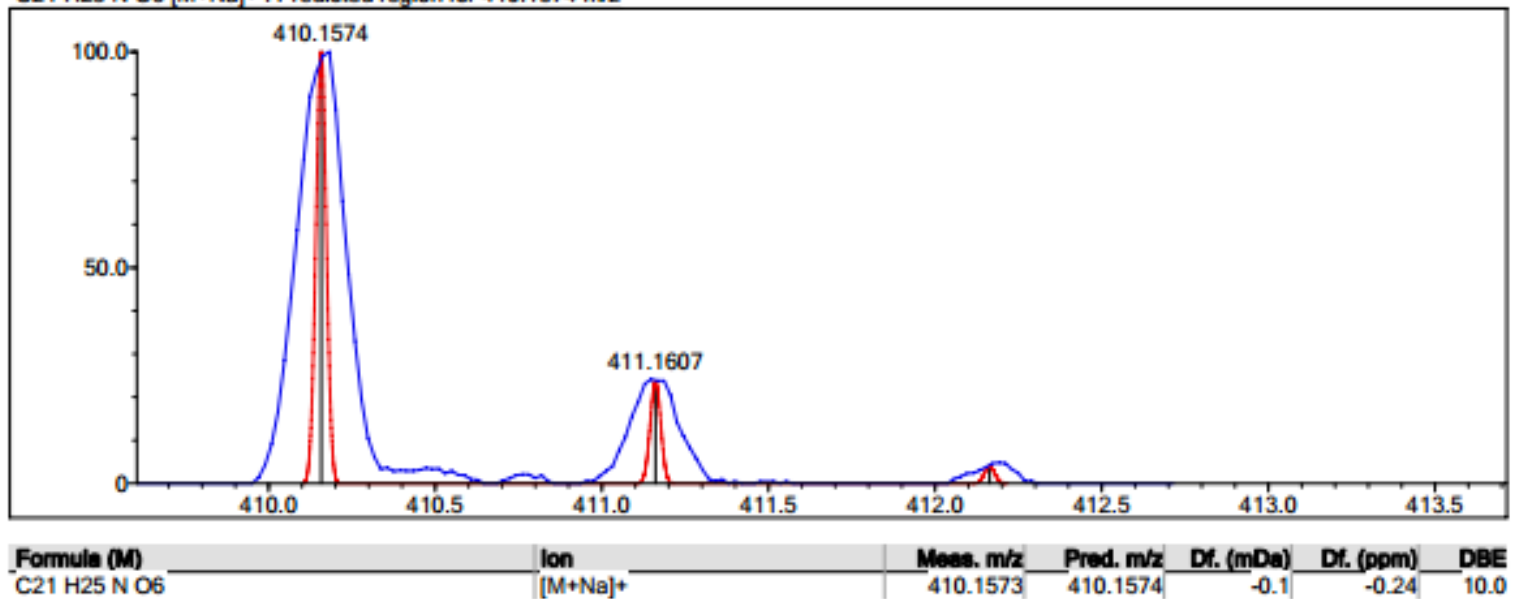



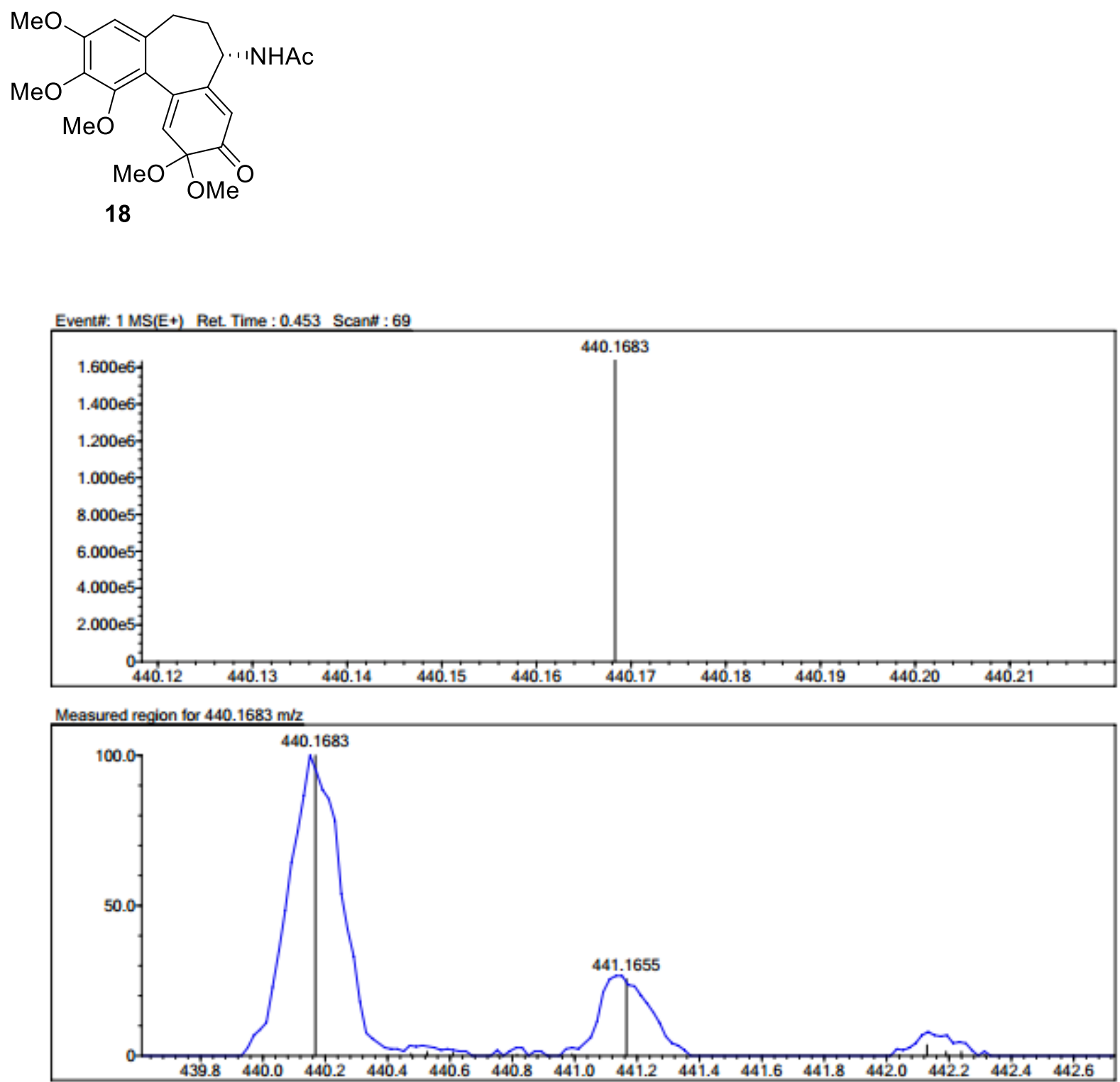

$\mathrm{C} 22 \mathrm{H} 27 \mathrm{~N} \mathrm{O} 7 \mathrm{M}+\mathrm{Na}]+$ : Predicted region for $440.1680 \mathrm{~m} / \mathrm{z}$

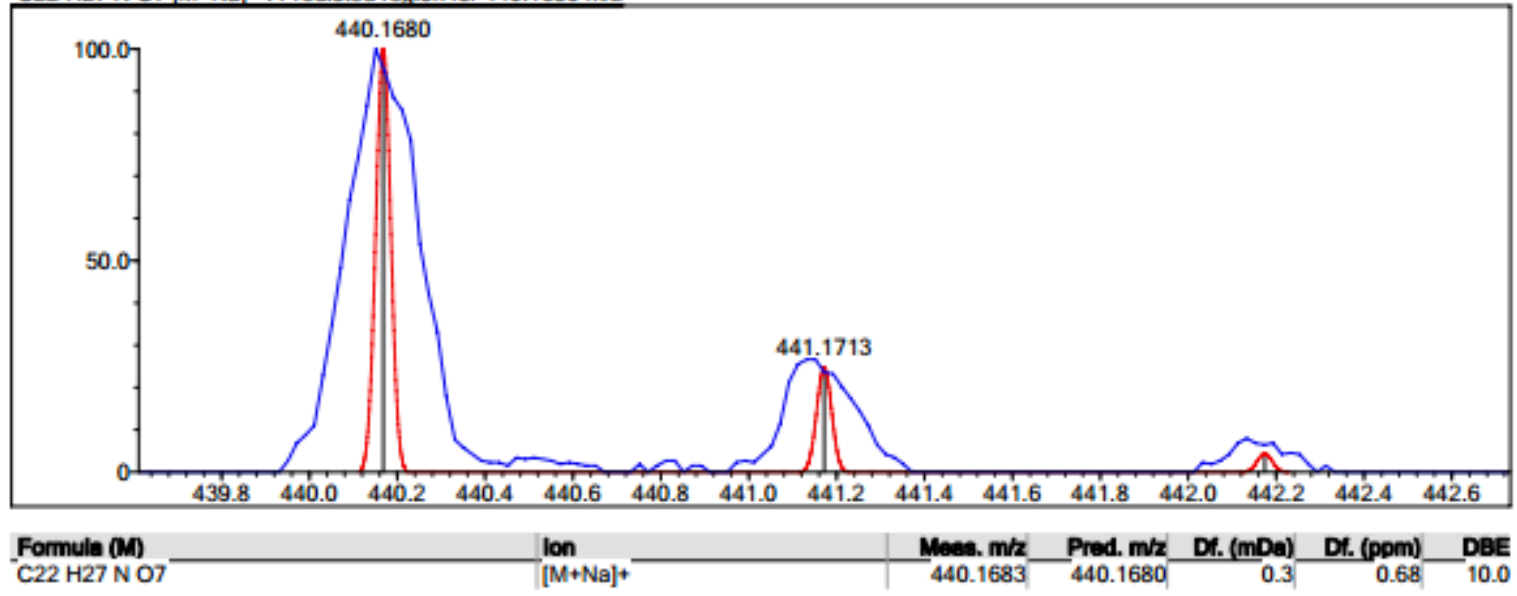




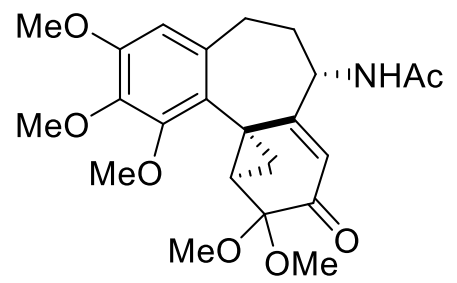

19
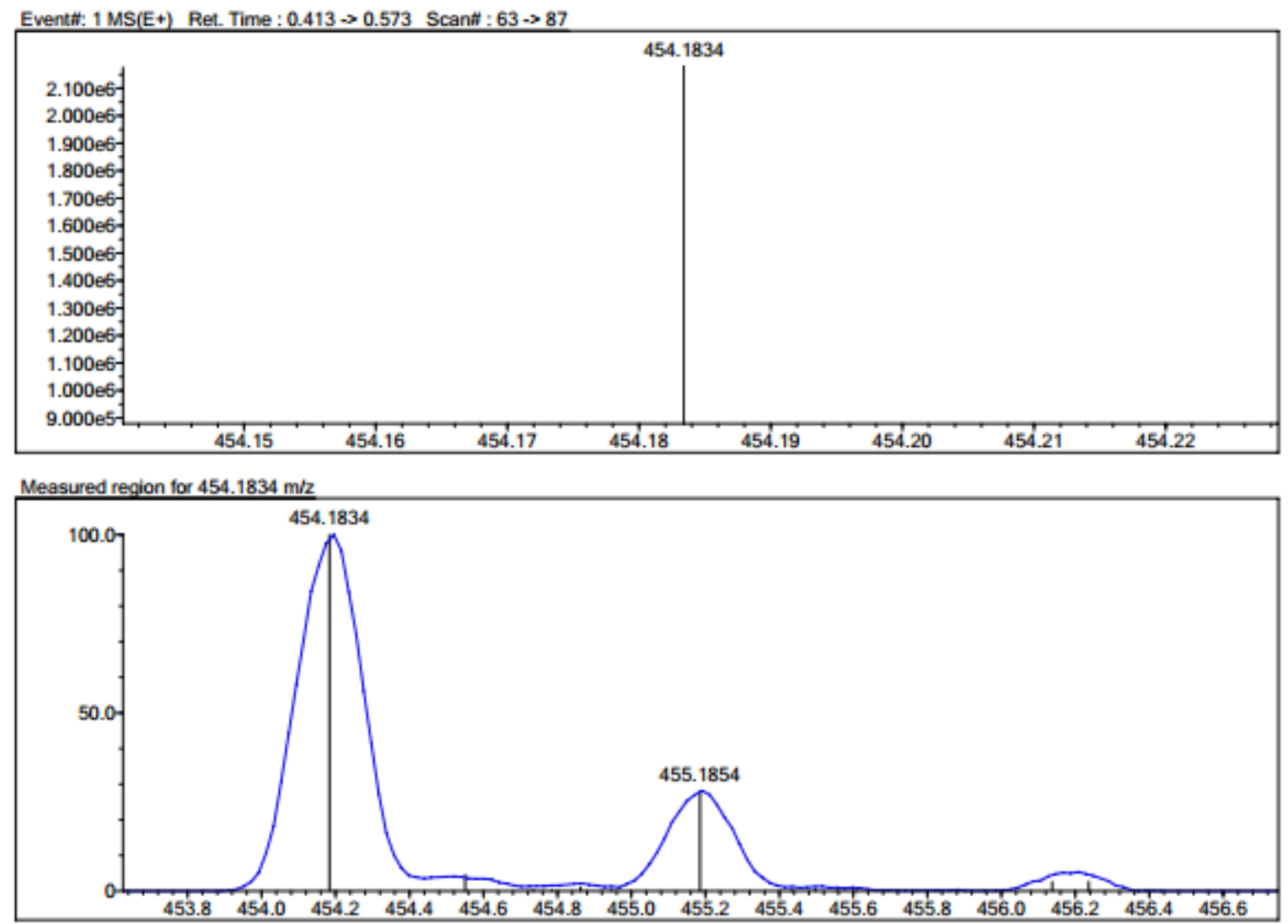

$\mathrm{C} 23 \mathrm{H} 29 \mathrm{~N} \mathrm{O} 7 \mathrm{M}+\mathrm{Na}+$ : : Predicted region for $454.1836 \mathrm{~m} / \mathrm{z}$

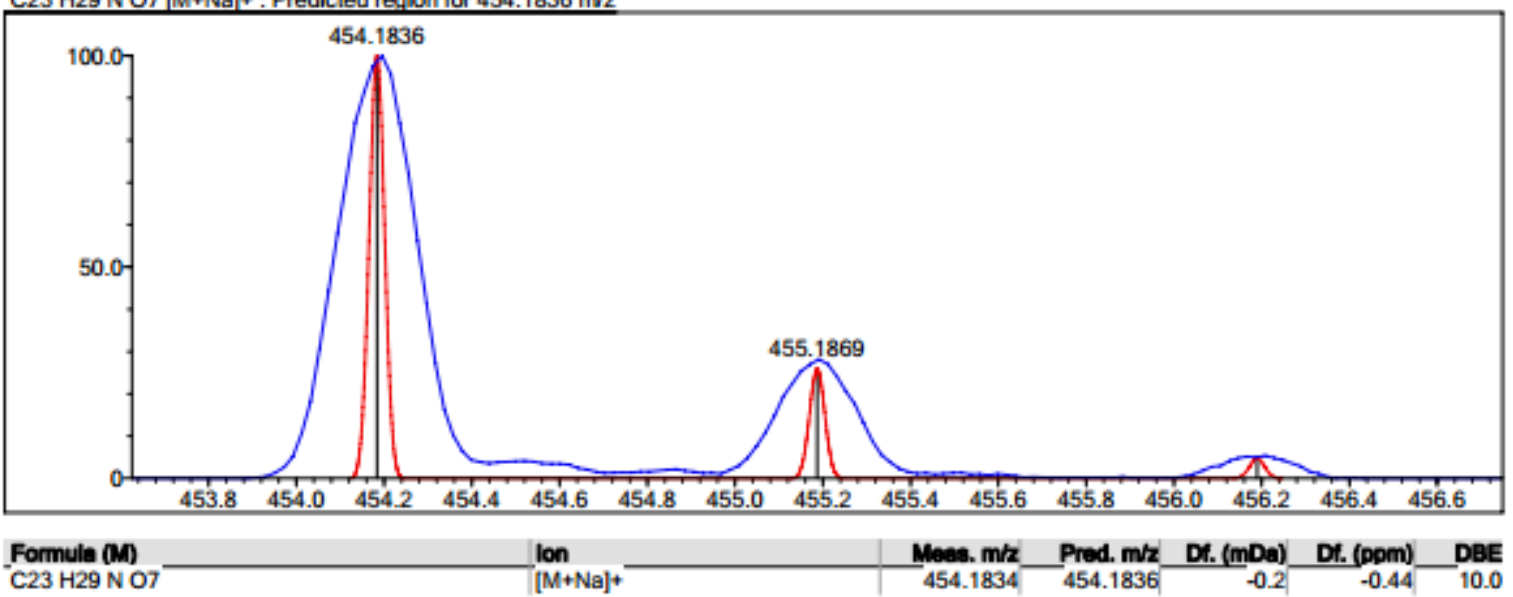


<smiles>CCCCCCCCNC1CCc2cc(OC)c(OC)c(OC)c2-c2ccc(OC)c(=O)cc21</smiles>

Eventt: 1 MS(E+) Ret. Time : $0.320 \rightarrow 0.373$ Scant : $49 \rightarrow 57$

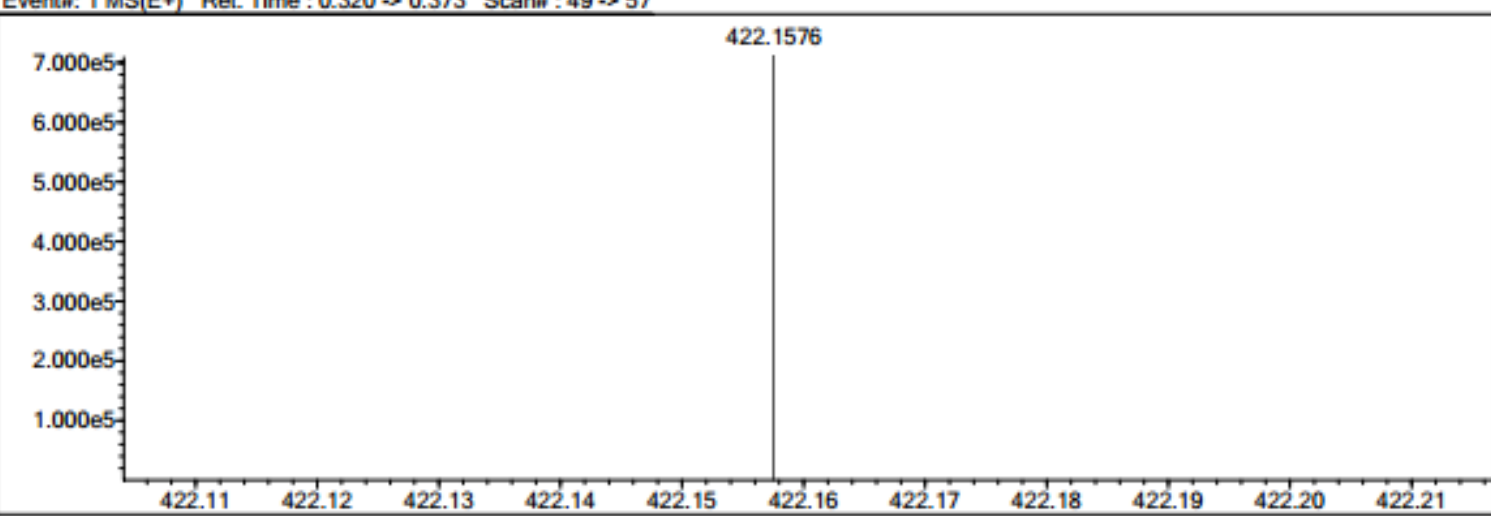

Measured region for $422.1576 \mathrm{~m} / \mathrm{z}$

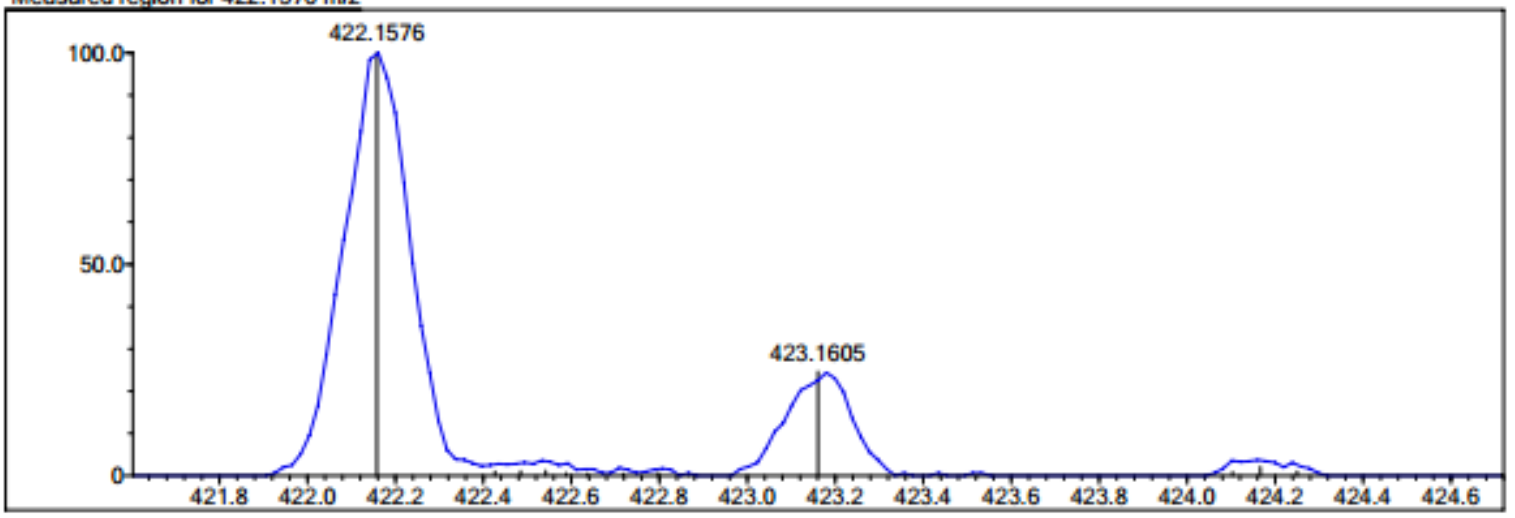

$\mathrm{C} 22 \mathrm{H} 25 \mathrm{~N}$ O6 [M+Na]+ : Predicted region for $422.1574 \mathrm{~m} / \mathrm{z}$

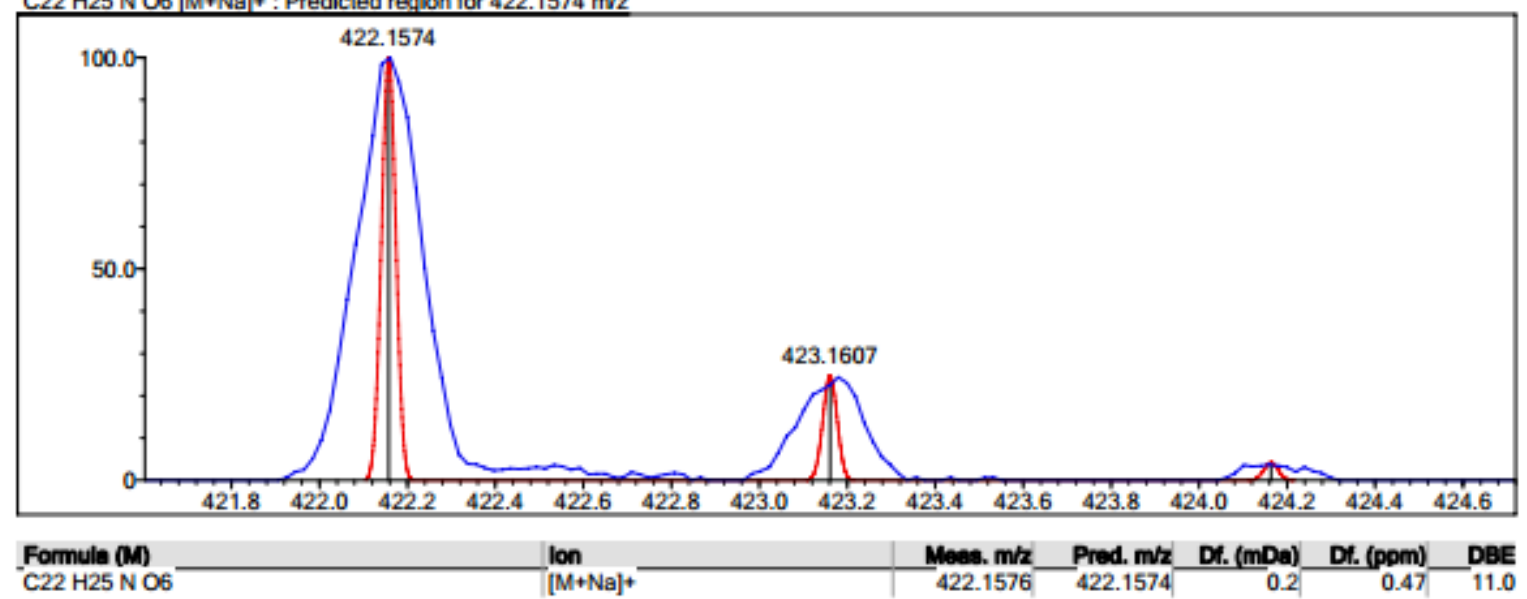


